University of South Florida

DIGITAL COMMONS

Digital Commons @ University of

@ UNIVERSITY OF SOUTH FLORIDA

South Florida

USF Tampa Graduate Theses and Dissertations

USF Graduate Theses and Dissertations

3-25-2004

\title{
Phase Imaging Digital Holography for Biological Microscopy
}

Daniel Parshall

University of South Florida

Follow this and additional works at: https://digitalcommons.usf.edu/etd

Part of the American Studies Commons

\section{Scholar Commons Citation}

Parshall, Daniel, "Phase Imaging Digital Holography for Biological Microscopy" (2004). USF Tampa Graduate Theses and Dissertations.

https://digitalcommons.usf.edu/etd/1191

This Thesis is brought to you for free and open access by the USF Graduate Theses and Dissertations at Digital Commons @ University of South Florida. It has been accepted for inclusion in USF Tampa Graduate Theses and Dissertations by an authorized administrator of Digital Commons @ University of South Florida. For more information, please contact digitalcommons@usf.edu. 
Phase Imaging Digital Holography for Biological Microscopy

by

\title{
Daniel Parshall
}

\begin{abstract}
A thesis submitted in partial fulfillment of the requirements for the degree of Master of Science Department of Physics College of Arts and Sciences University of South Florida
\end{abstract}

Major Professor: Myung K. Kim, Ph.D. Wei Chen, Ph.D. Dennis Killinger, Ph.D.

Date of Approval:

March 25, 2004

Keywords: biophysics, optics, hologram, microscope, cells

(C) Copyright 2004, Daniel Parshall 


\section{Dedication}

To my mother and father, who each, in their own very different ways, encouraged me to pursue the path of science. 


\section{Acknowledgements}

More than anyone else, Dr. Kim has been the driving force behind this work. It is his vision, his scientific integrity, and his quiet sense of humor that have provided the impetus for the pages you now hold in your hands. Nevertheless, I am also deeply indebted to James Gass, upon whose sweat and agony this work is built, and who took time to answer my asinine questions, and to discover the physics behind the experiments. Thanks are also due to Jack Huesman, for his ideas, and advice, and most unusually, for his remarkable patience with me as I worried out problems whose solution he saw in a moment 


\section{TABLE OF CONTENTS}

LIST OF FIGURES ii

ABSTRACT vi

I. INTRODUCTION

II. THEORY 6

$\begin{array}{lr}\text { A. Digital holography } & 6\end{array}$

B. Phase imaging digital holography 10

C. Notes on biological samples 13

III. APPARATUS 15

IV. PROCEDURE $\quad 21$

V.RESULTS 24

A. Low Magnification $\quad 24$

B. High-magnification imaging and intensity study 26

1. Onion cells $1 \quad 28$

2. Onion cells $2 \quad 39$

C. Multiple Wavelength PIH 46

1. USAF resolution target $\quad 47$

2. Onion cells $1 \quad 52$

3. Onion cells $2 \quad 57$

D. High resolution holography 62

1. USAF resolution target 63

2. Onion cells $1 \quad 67$

3. Onion cells $2 \quad 70$

4. Human blood cells $1 \quad 73$

5. Human blood cells $2 \quad 76$

$\begin{array}{ll}\text { VI. CONCLUSIONS } & 79\end{array}$

$\begin{array}{ll}\text { VII. REFERENCES } & 80\end{array}$

APPENDICES $\quad 82$

Appendix A (Comparison images) 83

Appendix B (Potential application) 85

Appendix C (Program documentation) 90

$\begin{array}{ll}\text { FOOTNOTES } & 104\end{array}$ 


\section{LIST OF FIGURES}

Fig. 1, Recording an off-axis hologram 2

Fig. 2, Retrieving an off-axis hologram 3

Fig. 3, Diffraction from hologram to reconstruction planes 6

Fig. 4, Mirror slanted with respect to incoming light 10

Fig. 5, Phase map of ramp using one wavelength 11

Fig. 6, Phase maps for two wavelengths $\quad 11$

Fig. 7, Subtraction of phase maps 12

Fig. 8, Photograph of experiment equipment 19

Fig. 9, Diagram of experiment apparatus 20

Fig. 10, Amplitude Image $(1.2 \times 1.2 \mathrm{~mm})$ - Onion cells 26

Fig. 11, Phase Image $(1.2 \times 1.2 \mathrm{~mm})-$ Onion cells 26

Fig. 12, Camera Response vs. Attenuation 28

Fig. 13, Direct image $(157 \mu \mathrm{m}, 360$ pixels per side $)$ - Onion cell 30

Fig. 14, Amplitude Image (157 $\mu \mathrm{m}, 360$ pixels per side) - Onion cell 31

Fig. 15, Phase Image (157 $\mu \mathrm{m}, 360$ pixels per side $)$ - Onion cell 32

Fig. 16, Amplitude Image (157 $\mu \mathrm{m}, 360$ pixels per side) - Onion cell 33

Fig.17, Phase Image (157 $\mu \mathrm{m}, 360$ pixels per side $)$ - Onion cell 34

Fig. 18, Amplitude Image (157 $\mu \mathrm{m}, 360$ pixels per side) - Onion cell 35

Fig. 19, Phase Image (157 $\mu \mathrm{m}, 360$ pixels per side $)$ - Onion cell 36

Fig. 20, Amplitude Image (157 $\mu \mathrm{m}, 360$ pixels per side) - Onion cell 37 
Fig. 21, Phase Image $(157 \mu \mathrm{m}, 360$ pixels per side $)$ - Onion cell

Fig. 22, Direct Image $(157 \mu \mathrm{m}, 360$ pixels per side $)$ - Onion cells

Fig. 23, Amplitude Image (157 $\mu$ m, 360 pixels per side $)$ - Onion cells

Fig. 24, Phase Image (157 $\mu \mathrm{m}, 360$ pixels per side $)$ - Onion cells

Fig. 25, Amplitude Image (157 $\mu$ m, 360 pixels per side $)$ - Onion cells

Fig. 26, Phase Image (157 $\mu \mathrm{m}, 360$ pixels per side $)$ - Onion cells

Fig. 27, Amplitude Image (157 $\mu$ m, 360 pixels per side $)$ - Onion cells

Fig. 28, Phase Image (157 $\mu \mathrm{m}, 360$ pixels per side $)$ - Onion cells

Fig. 29, Direct Image (157 $\mu \mathrm{m}, 360$ pixels per side) - USAF Target (HeNe 633)

Fig. 30, Amplitude Image (157 $\mu \mathrm{m}, 360$ pixels per side) - USAF Target G4E6 (Nd:YAG532)

Fig. 31, Phase Image (157 $\mu \mathrm{m}, 360$ pixels per side) - USAF Target 49 G4E6 (Nd:YAG 532)

Fig. 32, Phase Image (157 $\mu \mathrm{m}, 360$ pixels per side) - USAF Target G4E6 (HeNe 633)

Fig. 33, Compound Image ( $157 \mu \mathrm{m}, 360$ pixels per side) - USAF Target 50 G4E6 with $2 \pi$ ambiguities removed

Fig. 34, 3D Rotated Compound Image (157 $\mu \mathrm{m}, 360$ pixels per side) USAF Target G4E6 with $2 \pi$ ambiguities removed

Fig. 35, Direct Image (157 $\mu \mathrm{m}, 360$ pixels per side $)$ - Onion cells 52 (Nd:YAG 532)

Fig. 36, Amplitude Image ( $157 \mu \mathrm{m}, 360$ pixels per side $)$ - Onion cells (Nd:YAG 532)

Fig. 37, Phase Image (157 $\mu \mathrm{m}, 360$ pixels per side $)$ - Onion cells (Nd:YAG 532) 
Fig. 38, Phase Image (157 $\mu \mathrm{m}, 360$ pixels per side) - Onion cells (HeNe 54 633)

Fig. 39, Compound Image (157 $\mu \mathrm{m}, 360$ pixels per side $)$ - Onion cells 55 with $2 \pi$ ambiguities resolved (MWPIH)

Fig. 40, 3D Rotated Compound Image (157 $\mu \mathrm{m}, 360$ pixels per side $)-\quad 56$ Onion cells with $2 \pi$ ambiguities removed (MWPIH)

Fig. 41, Direct Image $(193 \mu \mathrm{m}, 472$ pixels per side) - Onion cells (HeNe 57 633)

Fig. 42, Amplitude Image (193 $\mu \mathrm{m}, 472$ pixels per side) - Onion cells 58 (Nd:YAG 532)

Fig. 43, Phase Image (193 $\mu \mathrm{m}, 472$ pixels per side) - Onion cells (Nd:YAG 532)

Fig. 44, Phase Image (193 $\mu$ m, 472 pixels per side) - Onion cells (HeNe 59 633)

Fig. 45, Compound Image (193 $\mu \mathrm{m}, 472$ pixels per side) - Onion cells 60 with $2 \pi$ ambiguities removed (MWPIH)

Fig. 46, 3D Rotated Compound Image (193 $\mu \mathrm{m}, 472$ pixels per side) Onion cells with $2 \pi$ ambiguities removed (MWPIH)

Fig. 47, Direct Image ( $88 \mu \mathrm{m}, 360$ pixels per side) - USAF Resolution 63 Target G7E4-6

Fig. 48, Amplitude Image ( $88 \mu \mathrm{m}, 360$ pixels per side) - USAF 64 Resolution Target G7E4-6

Fig. 49, Phase Image ( $88 \mu \mathrm{m}, 360$ pixels per side) - USAF Resolution 65 Target G7E4-6

Fig 50, 3D Rotated Image ( $88 \mu \mathrm{m}, 360$ pixels per side) with $2 \pi$ ambiguity - USAF Resolution Target G7E4-6

Fig. 51, Direct Image ( $88 \mu \mathrm{m}, 360$ pixels per side) - Onion cell 
Fig. 52, Amplitude Image $(88 \mu \mathrm{m}, 360$ pixels per side $)$ - Onion cell

Fig. 53, Phase Image ( $88 \mu \mathrm{m}, 360$ pixels per side) - Onion cell

Fig. 54, Direct Image ( $88 \mu \mathrm{m}, 360$ pixels per side), Onion cell with 70 nucleus

Fig. 55, Amplitude Image ( $88 \mu \mathrm{m}, 360$ pixels per side $)$ - Onion cell with 71 nucleus

Fig. 56, Phase Image ( $88 \mu \mathrm{m}, 360$ pixels per side) - Onion cell with 72 nucleus

Fig. 57, Direct Image (88 $\mu \mathrm{m}, 360$ pixels per side) - Human blood cells

Fig 58, Amplitude Image ( $88 \mu \mathrm{m}, 360$ pixels per side) - Human blood 74 cells

Fig. 59, Phase Image ( $88 \mu \mathrm{m}, 360$ pixels per side $)$ - Human blood cells

Fig. 60, Direct Image (88 $\mu \mathrm{m}, 360$ pixels per side) - Human blood cells 76

Fig. 61, Amplitude Image ( $88 \mu \mathrm{m}, 360$ pixels per side) - Human blood cells

Fig. 62, Phase Image ( $88 \mu \mathrm{m}, 360$ pixels per side) - Human blood cells 78

Fig. A1, Onion cell with nucleus (1000X)

Fig. A2, Onion cell, lysing nucleus (1000X)

Fig. A3, Onion cells, slightly decomposed (1000X)

Fig. A4, Onion cells, slightly decomposed (400X)

Fig. A5, Human blood cells (1000X)

Fig. B1, Reflection holography

Fig. B2, Optical path length through cells 


\section{Phase Imaging Digital Holography For Biological Microscopy \\ Daniel Parshall}

\section{ABSTRACT}

We apply the techniques of digital holography to obtain microscopic 3D images

of biological cells. Both the amplitude and phase images are obtained from a single hologram, with approximately $1 \mu \mathrm{m}$ lateral and $10 \mathrm{~nm}$ longitudinal

resolution. The results are combined with previous experiments resolving the $2 \pi$ ambiguity to produce continuous phase images of the samples. 


\section{INTRODUCTION}

Holography is a relatively recent interferometric technique. Like other such methods it relies on the interference of light to make measurements accurate to within a fraction of a wavelength; amplitude based techniques are limited by diffraction effects to the sizes above that of the wavelength. Although the foundations of holography were laid down by 1948 (Gabor [1]), it was not until the invention of the laser in 1960 that holography became truly viable. As early as 1962, high resolution holograms were being created (Leith, Upatnieks [2] ${ }^{1}$, and the first paper on holographic interferometry was published in 1965 (Powell, Stetson [3]). While computer generated holograms using Fraunhofer diffraction were first created in 1967 (Lohmann, Paris [4]), digital numeric reconstruction of imaged objects had not been accomplished until quite recently (Schnars, Thomas, Jueptner [5]).

The word holograph is derived from the Greek words holos, meaning whole, and graph, meaning picture. A hologram, therefore, is something that records all of the information available in a beam of light ${ }^{2}$; not just the amplitude, as in traditional photography, but also the phase of the light. The holographic process takes place in two stages: the recording of an image, and the retrieval.

In order to record most holograms, regardless of whether the information is recorded digitally or physically, a beam of light is used that is spatially coherent 
over long distances; in practical terms, this means the use of a laser. A laser beam is typically passed through a beam splitter, resulting in two beams. One of these is the object beam, which is shined on the object to be recorded, and the light scattered from the object illuminates the recording media (typically a photographic plate in physical holography, or a digital camera in digital holography). The other is the reference beam, which is shined directly on the recording media, producing an interference pattern as it interacts with the object beam. While it is possible for the reference beam to be parallel to the light scattered from the object, it is not necessary, nor does it need to be normal to the surface of the recording media. The recording process can be seen in Fig. 1.

Fig. 1, Recording an off-axis hologram ${ }^{3}$

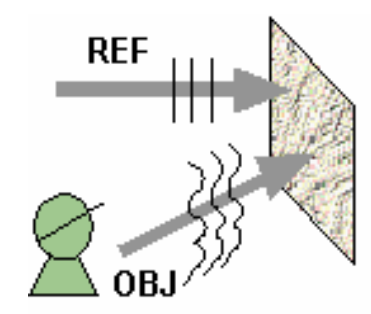

The image recorded is thus the amplitude of the combined reference and object beams. This can be represented as

$$
|H|^{2}=|O+R|^{2}=|O|^{2}+|R|^{2}+O * R+R * O
$$

where $\mathrm{H}$ is the intensity of the light recorded on a media, $\mathrm{O}$ and $\mathrm{R}$ are complex arrays of the form $E=A e^{i \theta}$, and the asterisk indicates the complex conjugate. 
The $|O|$ and $|R|$ are terms are the intensities of the object and reference beams, respectively, and the cross terms are the actual holographic contribution. While neither $O^{*} R$ or $R^{*} O$ are necessarily real, $O^{*} R+R^{*} O$ is always real. Thus, all the phase and amplitude information can be stored on real media.

To retrieve the holographic image in physical holography, it is necessary only to shine light upon the photographic plate, as in Fig. 2. By looking through the plate, a virtual image of the object can be seen that appears to have depth, and can be viewed at a wide range of angles. A real image is also produced, but is usually of minimal interest to us, as it is pseudoscopic (inside out). The virtual image corresponds to the $R^{*} O$ term, and the real image corresponds to the $O^{*} R$ term. The phase of the light in these cross terms is not an absolute phase, rather, it is the difference in phase with respect to the reference beam. This is significant because it requires us to use light of the original frequency in order to accurately retrieve the holographic data for interferometric purposes; again, a laser, with its extremely narrow bandwidth, is superior in this respect.

Fig. 2, Retrieving an off-axis hologram

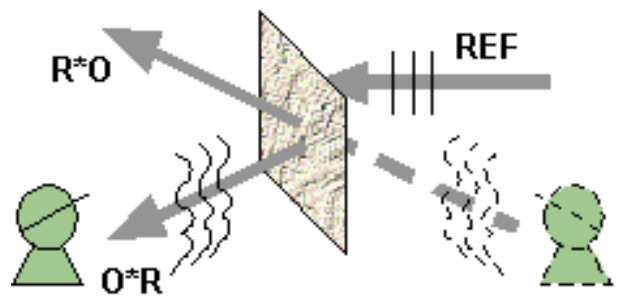


One of the most convenient aspects of digital holography is the ability to remove superfluous terms directly from the data set. This can be accomplished by imaging the hologram, the object beam alone, and the reference beam alone. The last two terms correspond to $|O|$ and $|R|$, the intensities of the object and reference beams. If these terms are subtracted from the hologram,

$$
\left|H^{\prime}\right|^{2}=|H|^{2}-|O|^{2}-|R|^{2}=O * R+R * O
$$

the only remaining terms are $O^{*} R+R^{*} O$, and the overall noise is reduced. This process can be extended to remove further terms, such as back scattering off of a lens. If the back reflection is $\mathrm{B}$, then the total light incident is $|H|^{2}=|O+B+R|^{2}$. Placing a blocker between the sample and L3 yields $|R+B|^{2}$; blocking the reference beam yields $|O+B|^{2}$; doing both yields $|B|^{2}$.

$$
\left|H^{\prime}\right|^{2}=|H|^{2}-|R+B|^{2}-|O+B|^{2}+|B|^{2}=O * R+R * O
$$

To retrieve the holographic image in digital holography, a computer calculates numerically what would be seen if light were to be shined upon the recorded hologram. The programming required is fairly simple; the reason digital holography is such a recent technique is because it is only lately that digital cameras capable of resolving the data required have become commercially available. The program requires that we treat each pixel of the hologram as a point source, and allow a spherical wave to propagate from each pixel, with the amplitude of the wave being proportional to the intensity of the light recorded on 
that pixel, in accordance with the Huygens wa velet principle (Schnars, Jueptner [6]; Kim [7]). As mentioned above, it is possible to have the reference and object beams parallel; this is referred to as an on-axis hologram. While such a situation is easier to calculate numerically, it has been found that the introduction of an angle between the object and reference beams results in an off-axis holograms; the $O^{*} R$ and $R^{*} O$ images become spatially separated ${ }^{4}[2]$. 


\section{THEORY}

\section{A. Digital holography}

The computation treats each individual pixel from the hologram as a point source; each pixel emits a spherical wave that propagates to another plane of pixels, the reconstructed image plane [6] [7]. Both planes are presumed to be the same size, and contain the same number of pixels. If there are $\mathrm{N}$ pixels per side in the holographic recording, it will consist of $\mathrm{N}^{2}$ pixels. Thus, each of the pixels of the reconstructed image will consist of $\mathrm{N}^{2}$ factors, one from each pixel of the holographic image. It is expected that a function of this type will be a convolution, since it examines the effect of every point in one function (the intensity of the holographic image) on each point of another function (the reconstructed image plane).

Fig. 3, Diffraction from hologram to reconstruction planes

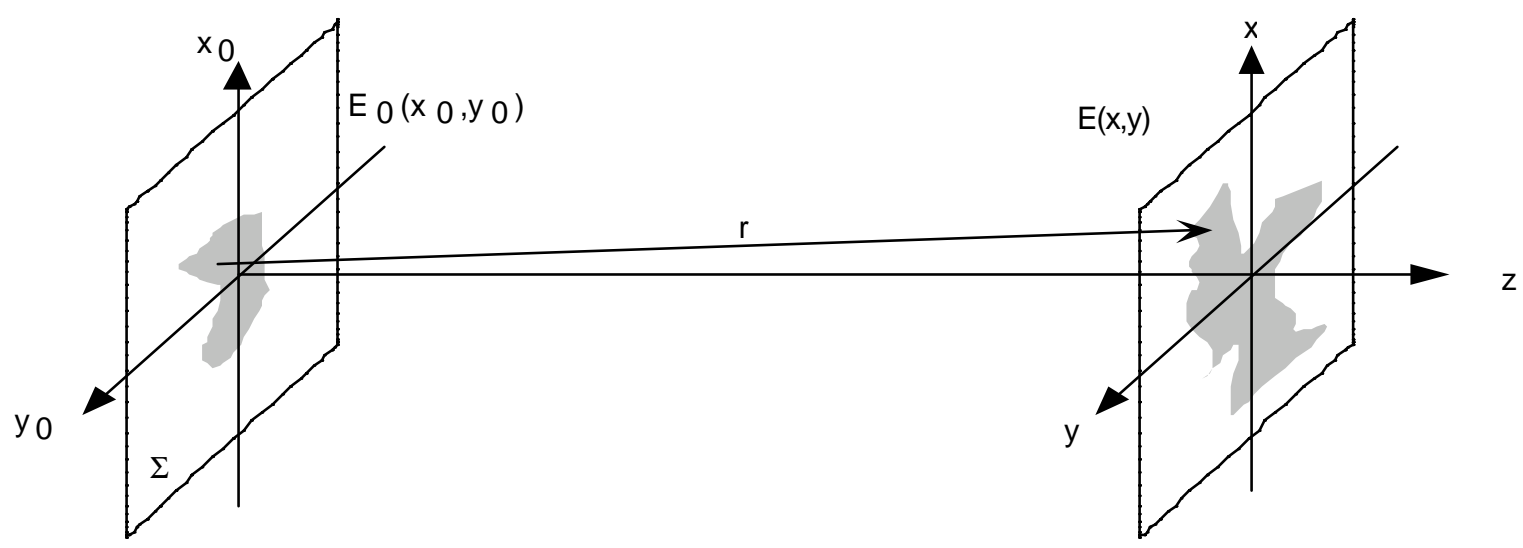


If the hologram is represented as a generalized 2 dimensional aperture, then each point of the holographic image plane is treated as a point source emitting a spherical wave with a intensity proportional to the brightness of the holographic image recorded at that point. This can be represented with a generalized 2 dimensional function of $x_{0}$ and $y_{0}$ in the holographic image plane, as seen in Fig. 3. If the distance between the holographic image plane $\left(x_{0}, y_{0}\right)$ and the reconstructed image plane $(x, y)$ is $z$, then the function takes the form

$$
E(x, y)=-\frac{i k}{2 \pi} \iint_{\Sigma} E_{0}\left(x_{0}, y_{0}\right) \frac{e^{i k r}}{r} d x_{0} d y_{0}
$$

where $E(x, y)$ is the field at the reconstructed image plane, $E_{0}\left(x_{0}, y_{0}\right)$ is the field at the holographic image plane, and $\frac{e^{i k r}}{r}$ represents a spherical wave being emitted from each point. The factor $r$ can also be written as

$$
r=\sqrt{\left(x-x_{0}\right)^{2}+\left(y-y_{0}\right)^{2}+z^{2}}
$$

making this substitution, and making the approximation $z \approx r$ in the denominator ${ }^{5}$, the equation becomes

$$
E(x, y)=-\frac{i k}{2 \pi z} \iint_{\Sigma} E_{0}\left(x_{0}, y_{0}\right) \exp \left[i k \sqrt{\left(x-x_{0}\right)^{2}+\left(y-y_{0}\right)^{2}+z^{2}}\right] d x_{0} d y_{0}
$$

The definition of a convolution in two dimensions is

$$
f \oplus g=\iint f\left(x_{o}, y_{o}\right) g\left(x-x_{o}, y-y_{o}\right) d x_{o} d y_{o}
$$

where the symbol $\oplus$ is used to represent the convolution. By comparing (2.3) and (2.4), it can be seen that the diffraction is simply a convolution of 
$E_{0}\left(x_{0}, y_{0}\right)$ with some function $S(x, y ; z)$; this function $\mathrm{S}$ is known as the Huygens point transfer function (PTF), where

$$
S(x, y ; z)=-\frac{i k}{z} \exp \left[i k \sqrt{x^{2}+y^{2}+z^{2}}\right]
$$

Thus,

$$
E(x, y)=\frac{1}{2 \pi} E_{o} \otimes S(x, y ; z)
$$

This relationship is of enormous benefit, since the convolution theorem from Fourier transform (FT) analysis shows that a convolution may be expressed as the inverse FT of the product of FTs. That is,

$$
f(x) \oplus g(x)=\mathfrak{I}^{-1}\{[\mathfrak{I}\{f(x)\} \times \mathfrak{I}\{g(x)\}]\}
$$

In this particular case, the function becomes

$$
E(x, y ; z)=\mathfrak{I}^{-1}\left\{\left[\mathfrak{I}\left\{E_{0}\right\} \times \mathfrak{I}\{S(x, y ; z)\}\right]\right\}
$$

So in order to reconstruct the image, it is necessary to perform merely 3 transforms: the FT of the hologram ${ }^{6}$, the FT of the PTF at the $z$ plane under consideration, and the inverse FT of the product of the two. With the advent of fast Fourier transform (FFT) algorithms, this process takes place in a matter of seconds ${ }^{7}$.

Because the number of pixels is finite, this becomes a discrete, as opposed to continuous, FT. In practice this means the use of an FFT. A few points must be considered when dealing with FFTs. The first is that the image quality will suffer if $\mathrm{N}$ is not sufficiently large. The second is that most FFT algorithms require the 
construction of matrices consisting $2^{\mathrm{K}}$ data points (Press, Flannerly, Teukolsky, Vetterling [8]); if the image has less than $2^{\mathrm{K}}$ data points, the remaining spaces are filled in with zeros by the FFT program. This means that there are certain "break points" when $N=\sqrt{2^{K}}$, and since the computation required is almost as intensive for a given value of $\mathrm{K}$, it is better to size $\mathrm{N}$ to its maximum value. Finally, an FT is evaluated over infinity. Since few scientists are willing to collect data until the end of time, the application of a "Fourier window" is required; "smooth" windows fade the value of the data being collected gradually to zero and are considered superior, as "sharp" windows introduce erroneous high frequency terms into the transform. This effect is known as aliasing; it also occurs when the sample rate becomes too low relative to the highest signal frequency in a band-limited data set [8].

In holographic imaging, aliasing can occur when the angle between the object and reference beams becomes too large; as the angle increases, the frequency of the interference fringes between the two beams increases. When the width of the fringes is shorter than 2 pixels, the solution for the computation includes erroneous low frequency terms because the higher frequency terms were not correctly sampled. For this reason, it is necessary to carefully consider how far off-axis the reference beam is set; moving the hologram off-axis improves quality as the $O^{*} R$ and $R^{*} O$ terms become separated. The quality increases until interference fringes begin to occur too frequently, when quality again goes down. 
The theoretical limitation for this to occur corresponds to the Nyquist frequency ${ }^{9}$, but experiments with the actual setup found it to be slightly lower than that.

\section{B. Phase imaging holography}

After performing the calculations, the result is a array of complex numbers that can be expressed in the form $E=A e^{i \theta}$. By taking the absolute value of any point in the array ( $A^{2}$, also referred to as the amplitude reconstruction), it is possible to find the intensity of the light at that point; the phase at that point is simply $\theta$ (the phase reconstruction). When viewing an object such as the sloped ramp depicted in Fig. 4, one would expect the amplitude of the light to remain essentially uniform across the surface. The phase is expected to vary as twice the height of the ramp, since the light must hit the mirror and bounce back.

Fig. 4, Mirror slanted with respect to incoming light

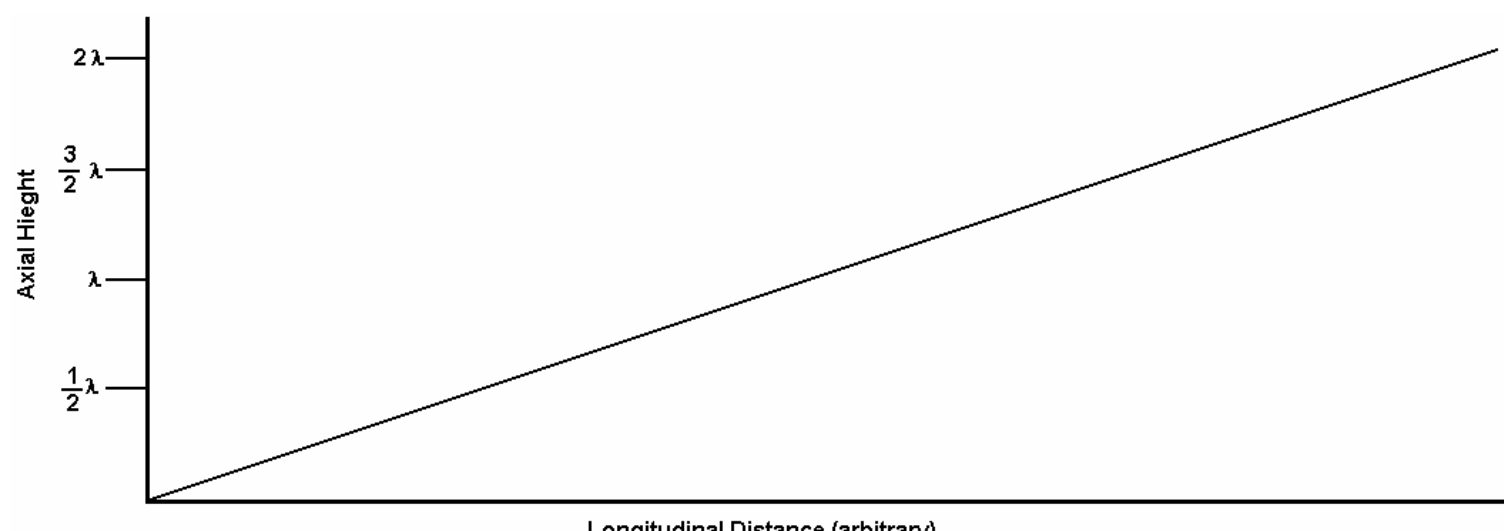


Fig. 5, Phase map of ramp using one wavelength

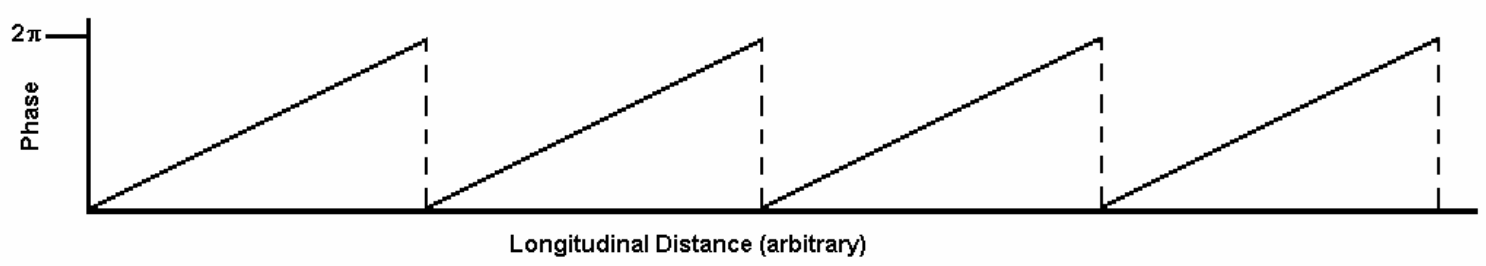

The resulting graph of the phase takes the form of a sawtooth wave, as seen in

Fig. 5. At every wavelength, there is a discontinuity in phase, since there is no method for directly distinguishing between 0 and $2 \pi$. Many phase imaging techniques resolve this ambiguity through complex algorithms, or by taking advantage of a priori knowledge of the structure of the object (Servin, Marroquin, Malacar, Cuevas [9]). Recent work in this laboratory has developed a technique that allows for the resolution of these ambiguities directly, by using two or more sources of light with different wavelengths (Gass, Dakoff, Kim [10]). By comparing the ambiguities in phase from each wavelength, the ambiguities can be resolved as if there were a single wavelength of light whose length is

$$
\Lambda_{12}=\frac{\lambda_{1} \lambda_{2}}{\left|\lambda_{1}-\lambda_{2}\right|}
$$

Fig. 6, Phase maps for two wavelengths

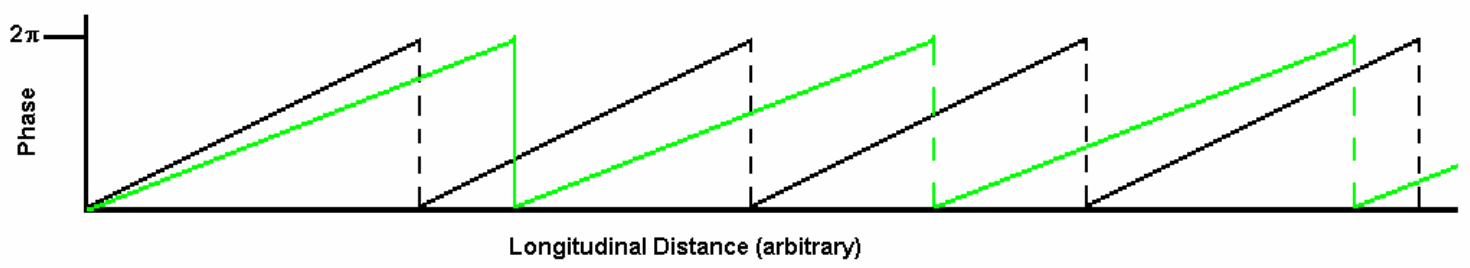


Multiple Wavelength Phase Imaging Holography (MWPIH) takes two separate phase images, as seen in Fig. 6. The algorithm for the unwrapping of phase discontinuities is simple; one wavelength is simply subtracted from the other, as seen in Fig. 7 . The result appears to be discontinuous, but by merely adding $2 \pi$ wherever the phase map is negative (addition modulo $2 \pi$ ), the result is a continuous slope that accurately recreates the original object.

Fig. 7, Subtraction of phase maps

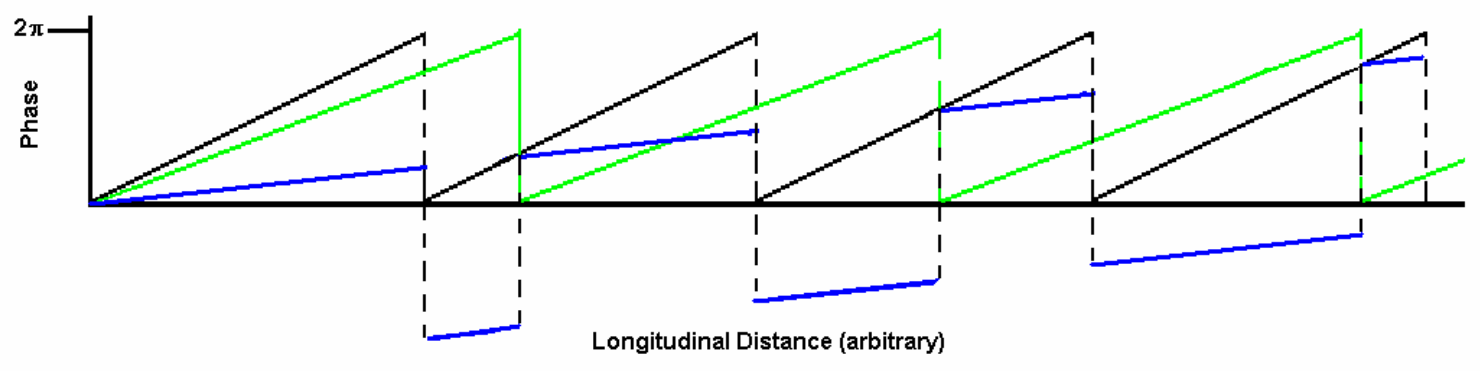

The total noise produced by this technique is initially the noise of the shortest wavelength multiplied by the ratio $\Lambda_{12} / \lambda_{1}$. However, this can be significantly reduced by using the coarse map as a guide, using the smallest available wavelength to map the surface, and adding factors of $2 \pi$ as indicated by the coarse map. The noise factor is presumed to be an uncertainty in phase, so the smallest wavelength corresponds to the smallest uncertainty in distance possible. The uncertainty value, as measured by other groups (Cuche, Bevilacqua, Depeursinge [11]) is approximately $10 \mathrm{~nm}$ rms for reflective surfaces using a single wavelength of $532 \mathrm{~nm}$. Previous experiments in this laboratory have 
established the same uncertainty value of $10 \mathrm{~nm}$ rms using multiple wavelengths, with $532 \mathrm{~nm}$ being the shorter of those wavelengths [10].

\section{Notes on biological samples}

The subject of this work is the application of the digital holography techniques described above to biological samples. The discussion up until now has been limited to reflection of light off the front surface of the object being imaged. When this is the case, the variation in height between two portions of a sample would be one half the difference as reported by the phase image. This remains true with biological images; the light passes through the sample, reflects off a mirrored surface, then passes through the sample again, so we expect the variation in height to be one half the value as measured by the phase of the light in this case as well.

However, the increased refractive index of the samples must be taken into consideration; we expect the variation in the height of the samples to be further reduced by a factor of the refractive index of the cellular material. Other researchers have established the value of this index to be between 1.333 to 1.436 for various plant cell walls (Gausman, Allen, Escobar [12]) and between 1.358 to 1.374 for mammalian cytoplasm (Lanni, Waggoner, Taylor [13]). These factors have not been taken into consideration when presenting these results. 
Finally, cells are dynamic. As a cell dies, it shrivels and begins to decompose. In some types of cells, the nucleus of the cell can drift to the outer membrane, break apart and spread its contents throughout the inside of the cell wall in a process known as lysing (Matthews [14]). In many of the early experiments, the cells used were, unbeknownst to the experimenter, already dead. These samples display classic characteristics of dead cells such as a rough and thick cell wall; this feature is caused by the shriveling membrane withdrawing from the cell wall [14]. In such cells the nucleus has already decomposed and is not locatable. Later experiments were conducted with fresh living cells. These cells have thin cell walls, clearly visible nuclei, and in some cases display features that may indicate the presence of further cellular organelles. 


\section{APPARATUS}

The apparatus for this experiment is effectively a modified Mach-Zehnder interferometer; one of the mirrors of the normal interferometer arrangement has been replaced with a beam splitter to allow illumination of the object. A photograph of the setup is shown in Fig. 8. A diagram of the experiment can also be found, as Fig. 9. Unless otherwise noted: all lenses used were achromatic to ensure that the paths did not diverge due to frequency dispersion when using both wavelengths; mirrors were dielectric coated, $\lambda / 10$ quality with $\mathrm{R}>99 \%$, selected based on their immediate availability; all beam splitters were dielectric coated wedge style, chosen because they produced less interference effects than pellicle or cube styles. The camera used was a Sony XC-ST50 8-bit monochrome camera, with $768 \times 494$ pixels over an area of $6.4 \times 4.8 \mathrm{~mm}$.

Not depicted in the diagram, but visible in the photograph, are a neutral density ${ }^{10}$ (ND) filter wheel (New Focus 5214) ranging from optical density ${ }^{11}$ (OD) 0.04 to OD 2.5. This is followed by a spatial filter (Newport 910), that uses a microscope objective of focal length $16.5 \mathrm{~mm}$, that focuses the laser beam onto a 15 micron pinhole. A $7.5 \mathrm{~cm}$ lens is used to collimate the output. The final beam diameter is adjusted as needed using a circular aperture; a typical diameter was approximately $1.5 \mathrm{~cm}$. 
Before entering the optical system, the two lasers used (Coherent $532 \mathrm{~nm}$ DPSS frequency doubled Nd:YAG and Coherent $633 \mathrm{~nm}$ HeNe gas laser) were steered towards a cube beam splitter to ensure they traveled along identical optical paths. This was the only place where a cube type beam splitter was used; the interference effects typically associated with such a component were negligible, since the beam splitter was located before the spatial filter. The high power output (approximately $400 \mathrm{~mW}$ ) of the Nd:YAG 532 required the use of a filter directly in front of the laser, with an effective OD 1.6, to reduce the input power to manageable levels (less than $100 \mathrm{~mW}$ ). The less powerful (approximately 80 $\mathrm{mW}) \mathrm{HeNe} 633$ laser did not require a filter of its own. Both lasers were adjusted from these initial powers by using the filter wheel mentioned above to bring their power to the range best suited for imaging.

The elements described above can be seen in the diagram, Fig. 9. The lasers were both steered to the cube beam splitter CBS, then through the spatial filter SF, then collimated using lens CL. This process ensured that the laser beams were perfectly parallel; arriving at the same point on CBS, the passing through SF selected only those portions of the wave front that were in identical directions.

After being cleaned and collimated, the laser beam arrived at BS1, where it would be split into object and reference beams. The reference beam entered $L 1$, and focused to a point F1, then passed on to BS3 where it was redirected to the camera. An ND filter was placed along the reference path if needed to adjust the 
relative intensity of the object and reference beams (not visible in the diagram). The object beam after leaving BS1 passed through L2, reflected off of BS2, and came to a focus at F2, which was the same optical distance from the camera as F1. The point F2 coincided with the back focus of L3, producing a collimated beam that was directed towards the object. The object was mounted on a translator equipped with a micrometer, allowing for accurate and repeatable displacement along the optical axis. After the beam was reflected off the target, it passed back through L3, through BS2, and through BS3, where it recombined with the reference beam to produce an interference pattern. Slight rotation of BS3 enabled the acquisition of off-axis holograms. Ideally, the fringe width produced by the interference between the object and reference beams would be equal to 2 pixels of the CCD camera.

In earlier, lower-magnification experiments, the CCD was fitted with an adjustable lens suitable for use in a high quality photographic or video system. The lens was set to focus (in conjunction with another lens not shown, L4) on the S plane of the system. In later, higher magnification experiments, the camera lens and L4 were removed from the system entirely, and the CCD was positioned so that the array itself was in the S' plane. The position of the S plane is somewhat arbitrary, provided that the camera is focused on the $S$ plane, and that the light scattering from the object is allowed to propagate a certain minimum distance that satisfies the approximation conditions used for the calculations. To ensure this criterion was met, the object was placed at an arbitrary point, its position as 
read by the micrometer recorded, and the camera system brought into focus; this plane is now the $\mathrm{S}$ plane. The object was then moved back along the axis at least the minimum required to the position where the object would be located during the actual imaging process, the I plane. The distance between the $S$ and I planes is Z.

In the case of biological samples, the scattering was quite high, and the use of a circular aperture A was found to significantly improve image quality. When the aperture was set smaller than the image to be acquired, the aperture acted as a physical Fourier window, fading the value of the scattering data to zero before reaching the start and stop points of the acquisition window, thus preventing noise introduction during the transform process. The largest effect, however, was simply that of keeping light from reaching the target anywhere except the spot being imaged, thus reducing the noise directly. To prevent fringing effects, the aperture was ideally positioned along the optical axis, at the image point of the object as seen through L3. In later, higher-magnification experiments, the physical size of the optical elements prevented the aperture from being located in this ideal position, but the effect of fringing is small compared to the benefits gained by reducing scatter from outside the sample area. Verification of aperture position was accomplished by first recording the position of the $S$ plane as measured by the micrometer, and focusing the camera onto the $\mathrm{S}$ plane. The object was then moved to the I plane, the camera focused on that plane, and the aperture was moved to ensure that the shadow cast by the aperture was as 
sharp as possible. The object was then repositioned in the $\mathrm{S}$ plane (as measured by the micrometer/translator) the camera brought to focus on the $S$ plane, and the object finally moved back into the I plane before acquiring data.

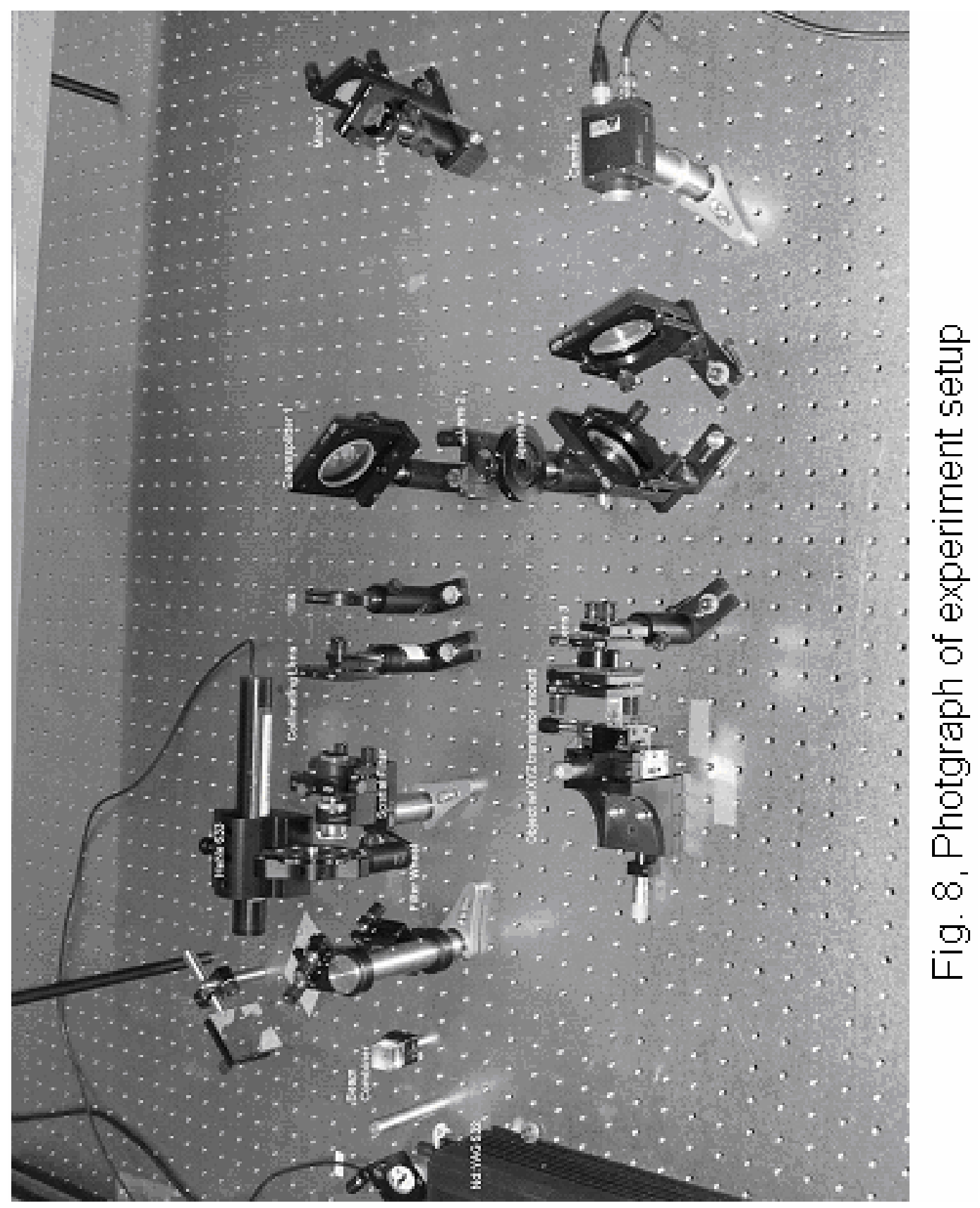




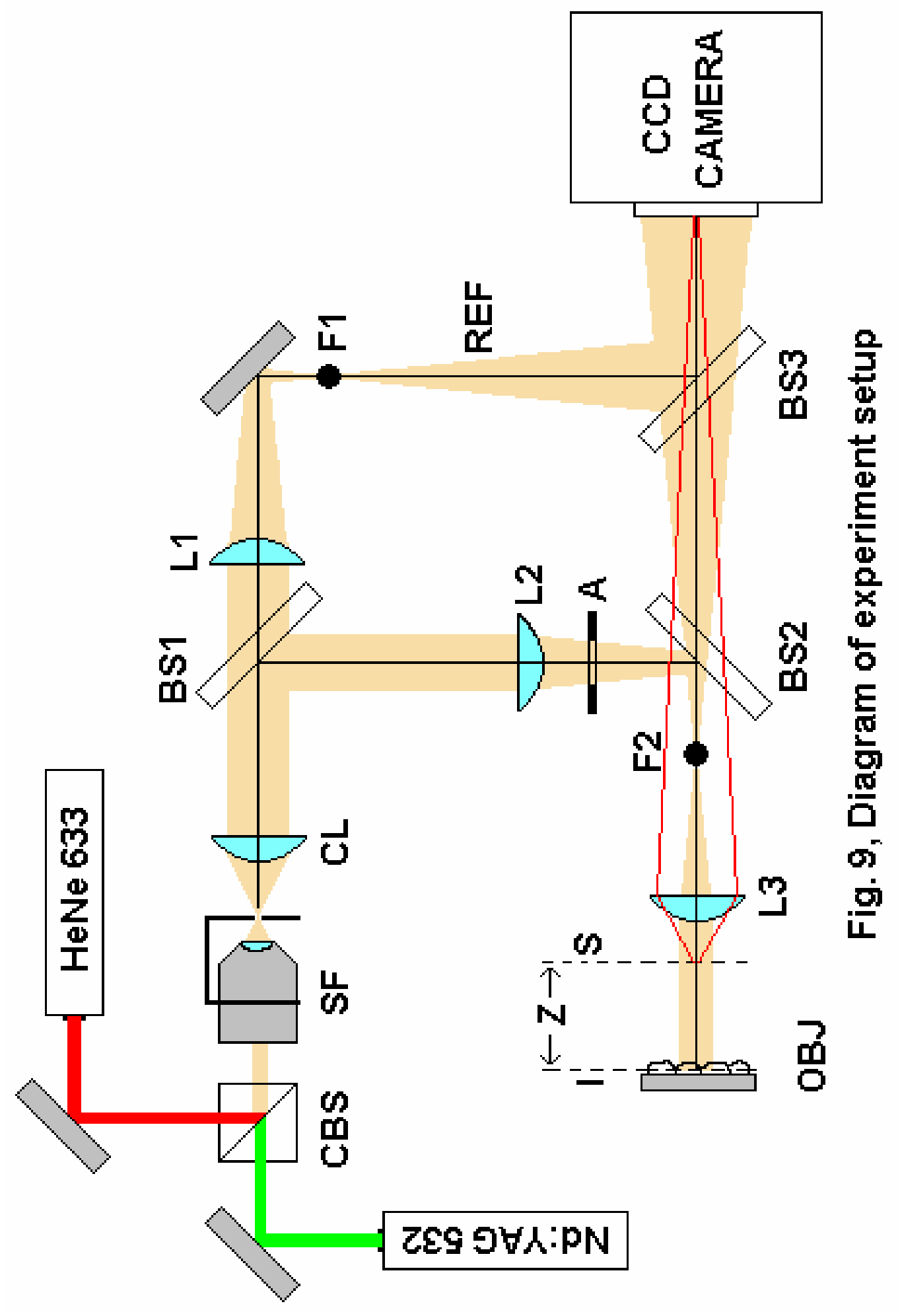




\section{PROCEDURE}

Holograms are obtained by turning on all lasers needed for the experiment and allowing them to warm up for a period of approximately 30 minutes. In the case of the Nd:YAG 532 laser, it has been found that the laser power may fluctuate from a nominal output of $400 \mathrm{~mW}$ to outputs as low as $300 \mathrm{~mW}$ once the laser has been warmed up. Briefly turning the laser off, then back on, eliminates this aberration. The HeNe 633 laser does not seem to be subject to power fluctuations.

After allowing the laser to stabilize, a sample is mounted on a mirror that is in turn mounted on the translator. The HoloExp program is then started, and the imaging subroutine, Hcamera, is called up. The sample is brought back and forth between the $S$ plane and the I plane to ensure that the mirror is mounted perfectly perpendicular to the optical axis. Once this is verified, the sample is brought into focus, and a section of the sample that seems to have clearly identifiable features such as cell walls or nuclei is selected for imaging. The target is then moved back to the I plane, as measured by the micrometer. The histogram of pixel intensity is then viewed, and various parameters, such as the ND filter wheel, reference beam ND filter, and the black and white reference voltages for the CCD camera are adjusted to optimize the image quality. 
The Hcamera subroutine is exited, and the hologram image is acquired to memory by selecting "TakeH". Immediately afterwards, the $\mathrm{O}^{*} \mathrm{O}$ and $\mathrm{R}^{\star} \mathrm{R}$ terms are removed from the hologram by blocking the reference and object beams, respectively, and clicking "SubtOR". If needed, "AddB" will add an image. The data acquisition process is now complete, and an array is now stored in the computer's memory of the $\mathrm{O}^{*} \mathrm{R}$ and $\mathrm{R}^{\star} \mathrm{O}$ terms.

The Diffract subroutine is entered, allowing the user to adjust various parameters as inputs of the FFT routine, which is handled in MatLab. Reasonable starting parameters can be estimated from a few factors; the $Z$ value used for the optimal image quality is often quite close to the actual distance between the $S$ and I planes. After all of the parameters have been adjusted to produce the highest quality image, the Diffract subroutine is closed, and the data and associated parameters are saved from the top level program (HoloExp) in a variety of formats $\left({ }^{*} . t x t,{ }^{*} . b m p,{ }^{*} \mathrm{cbn}\right.$, etc.). At this point, the entire process is repeated for the next wavelength.

Once all the data has been obtained for the various wavelengths, the PIH program is opened. Upon starting the PIH program, the files containing the arrays of complex numbers are opened. On each of the two panels visible in the PIH program, the user can choose to display either: the height as calculated from first or second wavelength, or the coarse map generated by the beat wavelength, or from the first wavelength with the ambiguities resolved. The user can also 
adjust a variety of parameters, from shifting one of the images so they align correctly (although when care is taken aligning the two laser beams, this feature is not necessary), to adding or subtracting a phase constant uniformly throughout the arrays. In this way the user can easily confirm what they are seeing, and adjust these parameters to yield optimum image quality. 


\section{RESULTS}

This section includes the results for all of the various experimental setups. In this section, direct image refers to an image acquired while the target was located in the S plane; this is presumably the best resolution the system can achieve of that sample. Amplitude image is the calculated array showing only the $A^{2}$ values. The phase image shows only the $\theta$ values. Compound image refers to the composite image created by using two phase images to eliminate the $2 \pi$ ambiguities. Rotated 3D image is an image that has been rendered floating in space, and can be useful to see details of another image. All images are square, with the size given in $\mu \mathrm{m}$ and pixels. For comparison, images of onion and blood cells acquired with a digital microscope are located in Appendix A.

\section{A. Low-magnification biological imaging}

The first set of experiments were conducted at low power magnification, imaging a square $1.2 \mathrm{~mm}$ per side. In this series of experiments, a $3 \mathrm{~cm}$ achromatic triplet lens (diameter $25 \mathrm{~mm}$ ) with numerical aperture ${ }^{12}$ (NA) approximately 0.39 , Edmund Optics 45,211 was used as the L3 objective lens, in conjunction with a $20 \mathrm{~cm}$ lens for $L 4$, and the adjustable camera lens. The goal of these early experiments was primarily to test the validity of this method of imaging as applied to biological samples. Onion cells were selected as the sample to be used, as 
their size and structure are well established and easily identifiable; they are also one of the largest size cells that are readily available.

The first set of these experiments were conducted under the assumption that light was reflected from the front surface of the cells. The cells were mounted onto a black background of fine carbon particles that had an extremely low reflectivity. While it was possible to obtain direct images of the cells, it was not possible to obtain a holographic reconstruction. These experiments were conducted using a piece of the outer layer of onion skin, very thin and already dried, but also fairly reflective.

The samples were then mounted directly onto a mirror. This was done in order to take advantage of the translucent quality of the cells. Allowing the light to pass through the cells and reflect off the mirror ensured that a high percentage of light passed through the sample coherently; the surface profile is formed from the increased refractive index of the sample. The samples were mounted by cutting into the surface of the fresh onion material, and peeling off a layer of the outermost cells. The sample was then moistened, and applied directly to the mirror, with the water providing sufficient cohesion to hold the sample in place. These were the first biological samples to be successfully imaged with the digital holography technique. The structure of the cells was easily discernable in the amplitude reconstruction. In most of the images, the structure was not nearly as 
clear in the phase reconstruction, but the results nevertheless were encouraging, and provided impetus to proceed to higher magnification experiments.

Fig. 10, Amplitude Image (1.2 x $1.2 \mathrm{~mm})$ - Onion cells

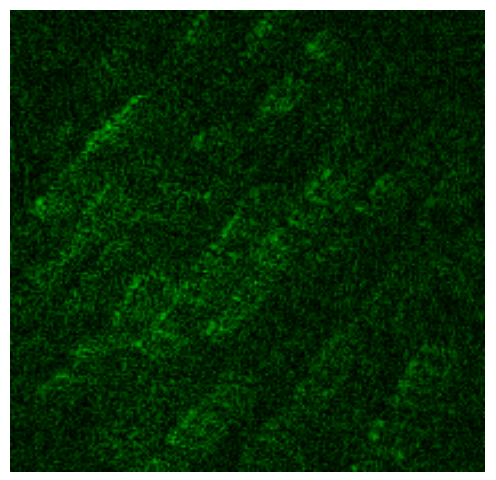

Fig. 11, Phase Image $(1.2 \times 1.2 \mathrm{~mm})-$ Onion cells

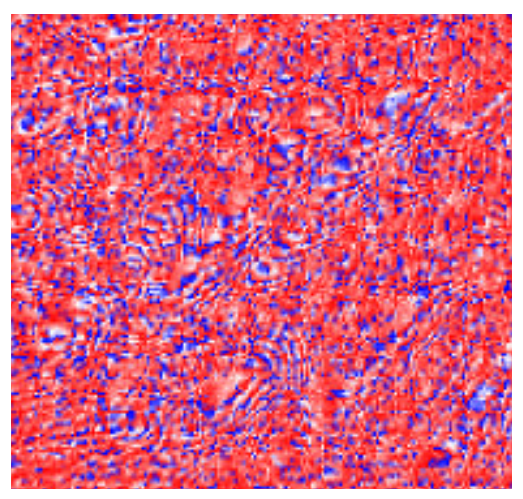

B. High-magnification imaging and intensity study

The experimental setup was modified to take higher magnification images. This was done by replacing L3 with an achromatic, anti-reflection coated lens of focal length $1.5 \mathrm{~cm}$ (6.25 mm diameter) and NA 0.2, Edmund Optics NT08-044. The adjustable lens was removed from the camera, L4 was removed entirely, and the 
camera was situated so that the CCD array was directly in the S' plane. These results were presented at the Optics In the South East conference in November 2003 (Parshall, Kim [15]).

Using this setup, a simple experiment was performed to check the linearity of the camera response. Linear response is desirable, but not required; non-linear response produces attenuation of the amplitude image, but the phase information remains unchanged (Goodman [16]). The response of the CCD camera was measured by writing a program that inferred the intensity by measuring the strength of the signal caused by the incident light by using the equation

$$
\text { Intensity }=\frac{\sum_{k=0}^{255} n_{k} V_{k}}{N^{2}}
$$

where $k$ represents each possible recorded intensity value $(0-255), V_{k}$ is the voltage required to generate that value ${ }^{13}, n_{k}$ is the number of pixels with that value, and $\mathrm{N}$ is the number of pixels per side. Using this equation, intensity measured in volts/pixel $(\mathrm{V} / \mathrm{p})$.

The test was performed using the lowest attenuation setting of the filter wheel (OD 0.04) as a baseline, and comparing the actual camera response to increasing attenuation levels (OD $0.5,1.0,1.5,2.0,2.5)$ to the theoretical value for a perfectly linear response. We expect that if the OD increases by 1 , the signal received should decrease by a factor of 10 . The data is displayed on a semi-log graph, with optical density along $x$-axis and intensity (measured in volts 
per pixel, using the equation above) displayed along the y-axis. The ambient noise value, found by blocking all light to the camera and recording the intensity measured by the camera, was subtracted from the values measured. This approximation is fairly reasonable, as the black reference voltage was usually set higher than that required to remove the ambient noise component. The results are displayed in Fig. 12.

Fig. 12, Camera Response vs. Attenuation

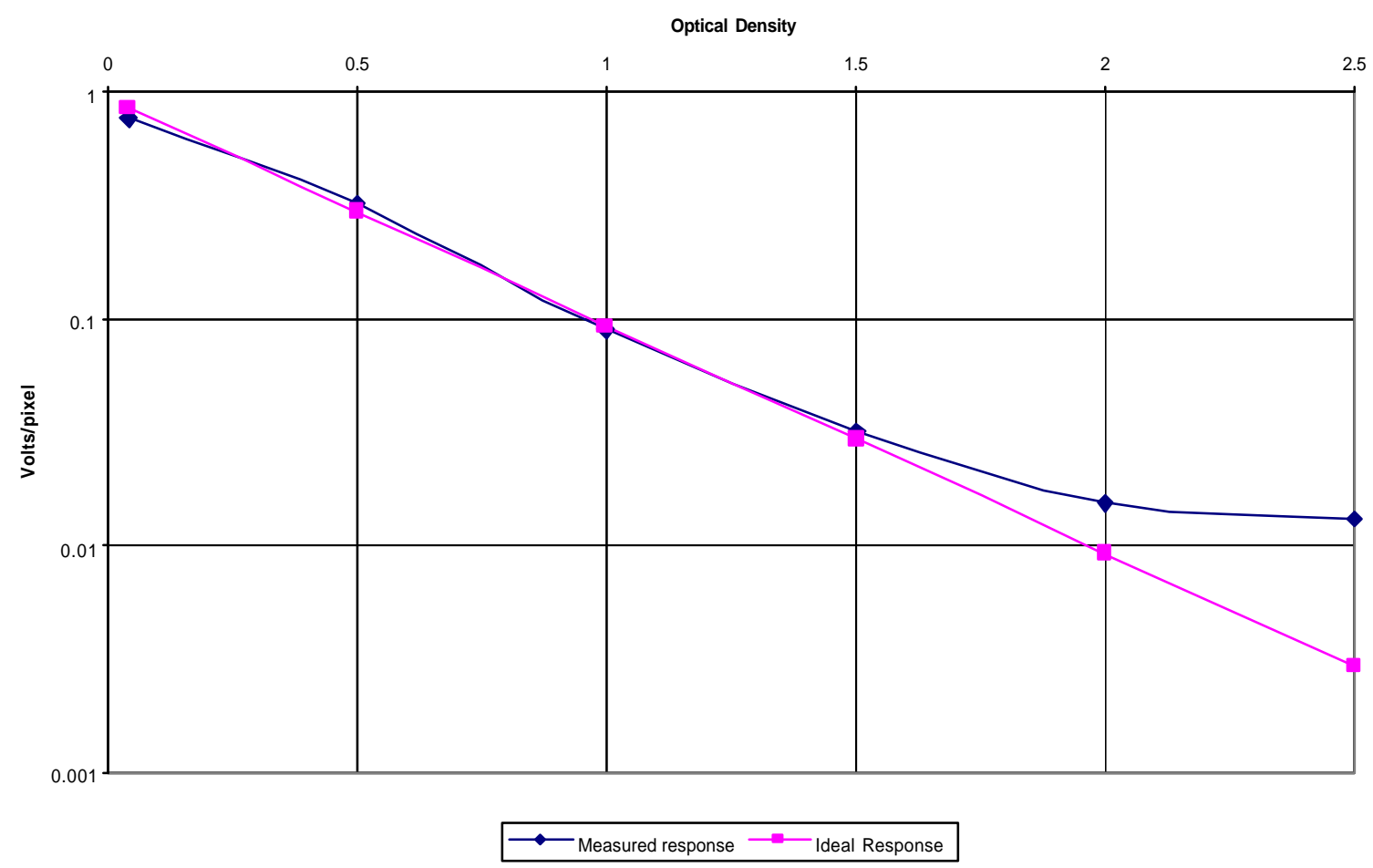

The high degree of linearity for stronger intensities is encouraging, and implies that reasonably strong illumination should be used when possible. Direct observational experiments suggest that the reference beam should be significantly brighter than the object beam. Additionally, images with clearly 
discernable features were acquired with a very dim object beam intensity and low overall intensity; whereas extremely bright illumination often produced fairly noisy phase images. This indicates that the benefits of a narrow signal range surpass those of linearity, which may be due partly to the fact that the reference beam tends to have a narrow range of intensity values; the object beam typically has a wide range of intensity values. Nevertheless, the technique works quite well under a broad range of parameters, as seen below.

\section{High-magnification holography - onion cells}

A number of holograms were acquired, spanning a broad range of experiment conditions such as: object distance from the focal plane; ratio of object to reference beam; overall light intensity; and inclusion of an aperture. It was discovered that there are a broad range of parameters that can produce good quality images, which is an encouraging indication of the robustness of this technique. All samples were squares $157 \mu \mathrm{m}, 360$ pixels per side. The minimum distance from the focal plane $\left(Z_{\min }\right)$ was $130 \mu \mathrm{m}$. The approximate intensity of the object and reference beams (OBJ and REF) over the imaged area is given in Volts/pixel (V/p). All of the data in this section was acquired using the sample of onion cells seen below. In the first data set (a) the object beam was roughly half as bright as the reference beam and $Z=2 Z_{\min }$, a fairly standard set of conditions.

In set (b) the $Z$ and overall light were kept the same, but the object beam was strengthened. In (c) the $Z$ was kept the same, but the overall light level was 
increased as well. In (d) the overall intensity and the ratio between the beams was returned to normal, but the $Z$ distance was cut to one half the minimum.

Fig. 13, Direct image $(157 \mu \mathrm{m}, 360$ pixels per side $)$ - Onion cell

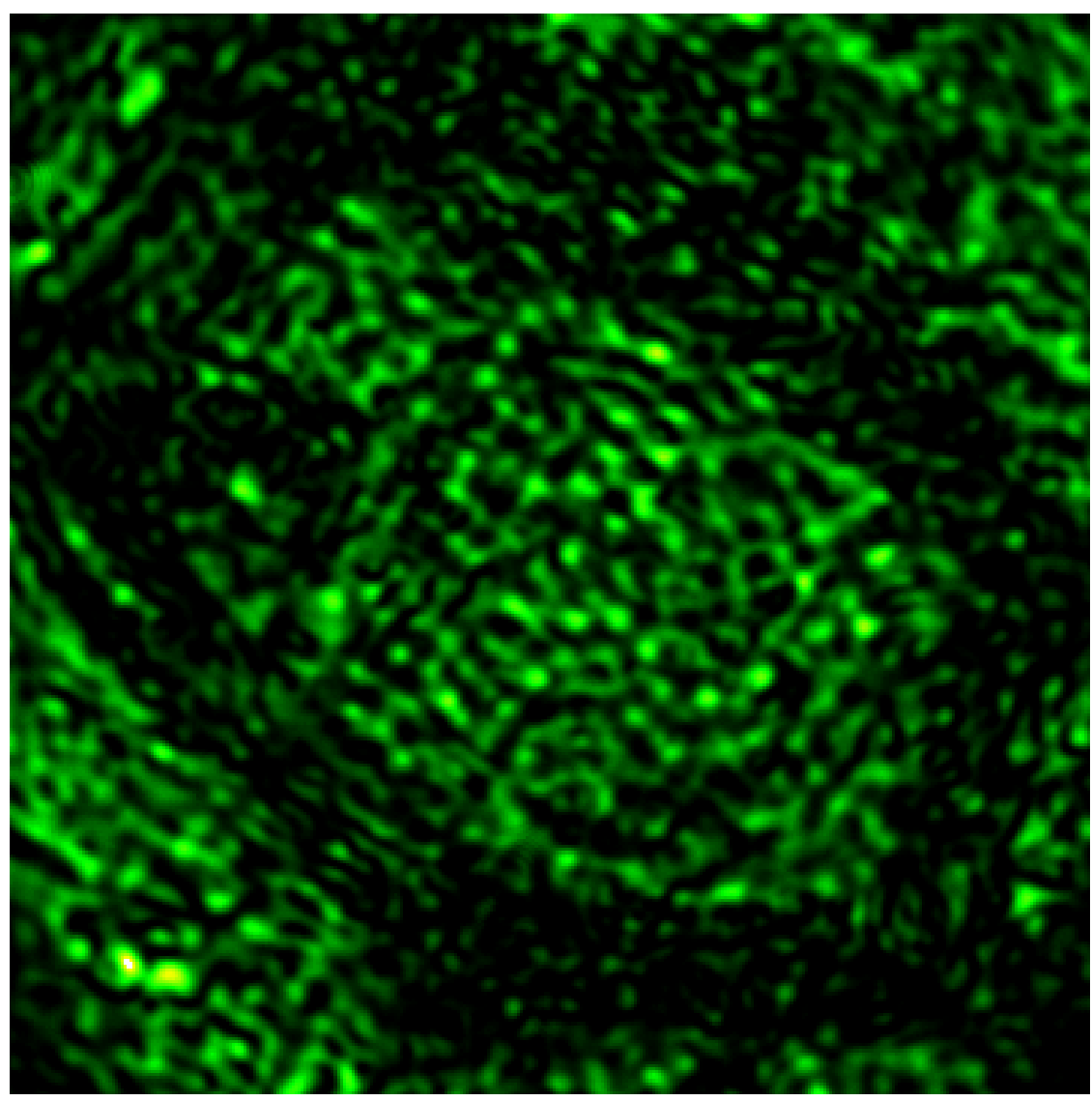

Most of the central region is occupied by a single cell surrounded by a thick cell wall. The upper right and lower left corners also have cells visible. In the extreme corners, the edges of the shadow cast by the aperture can just be seen. 
(a) Onion cells 1 - with aperture, OBJ $0.12 \mathrm{~V} / \mathrm{p}, \mathrm{REF} .23 \mathrm{~V} / \mathrm{p}$

Fig. 14, Amplitude Image (157 $\mu \mathrm{m}, 360$ pixels per side) - Onion cell

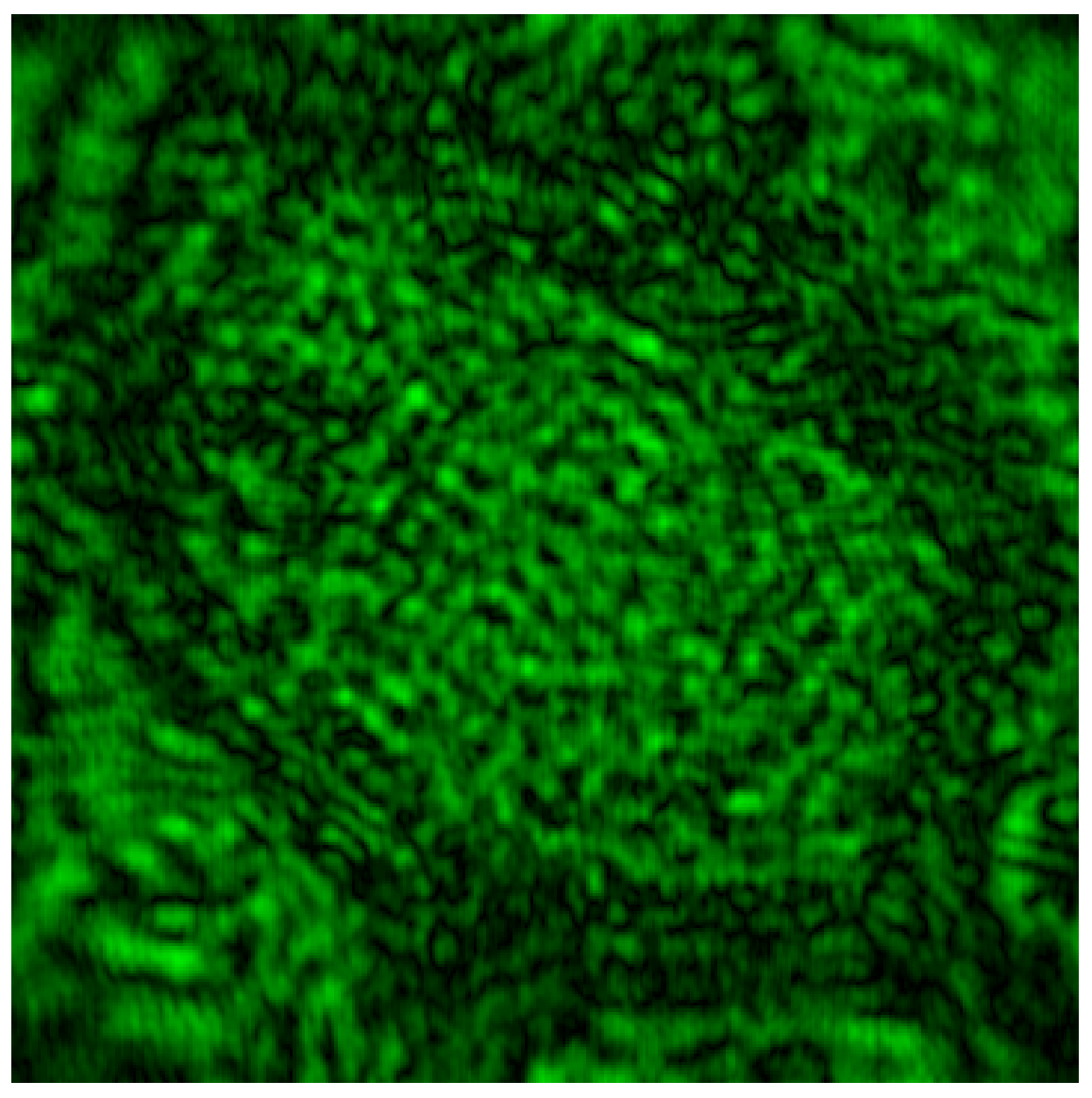

In this reconstruction of the cell seen in Fig. 13, the standard parameters were used of object to reference beam ratio of about 1:2. The object was imaged about $250 \mu \mathrm{m}$ from the $S$ plane. The main body of the central cell is clearly visible, and the cells in the upper right and lower left corners can still be seen. The distortion around the outer corners is partly caused by the fringes from the aperture shadow, as the aperture could not be placed in the ideal position during these experiments due to the size and distance requirements of the other components. 
Fig. 15, Phase Image $(157 \mu \mathrm{m}, 360$ pixels per side $)$ - Onion cell

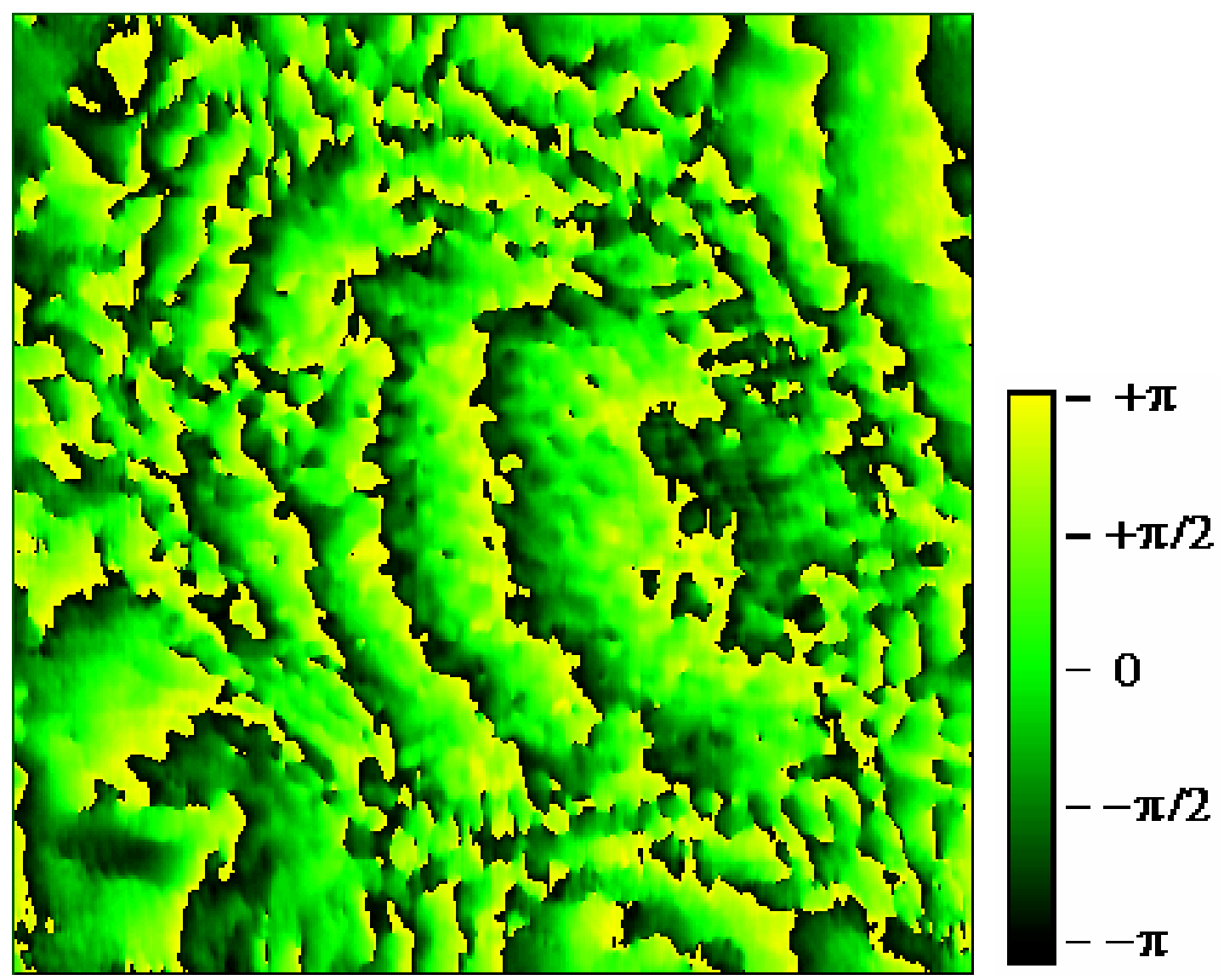

This is a fairly good example of a typical phase image. The main body of the cell appears fairly smooth and features several examples of the $2 \pi$ ambiguity that shall be resolved in the next series of experiments (see page 46). The cell walls appear as very rough areas in the phase images. 
(b) Onion cells 1 - with aperture, OBJ $0.15 \mathrm{~V} / \mathrm{p}, R E F .25 \mathrm{~V} / \mathrm{p}$

Fig. 16, Amplitude Image (157 $\mu \mathrm{m}, 360$ pixels per side) - Onion cell

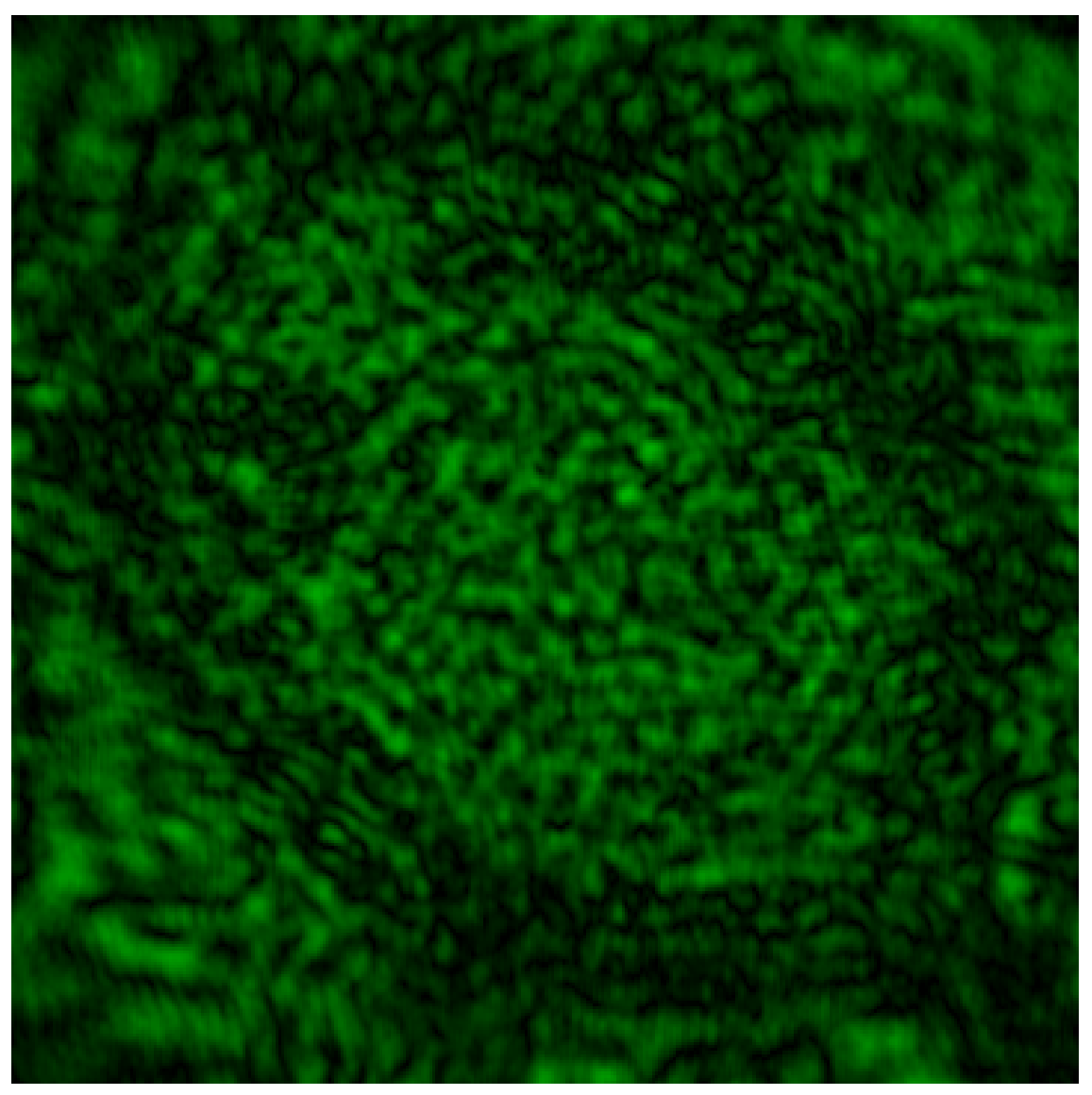

In this amplitude reconstruction, the cell appears fairly similar to the direct image, even though the ratio between object and reference beams has been increased. 
Fig.17, Phase Image $(157 \mu \mathrm{m}, 360$ pixels per side $)$ - Onion cell

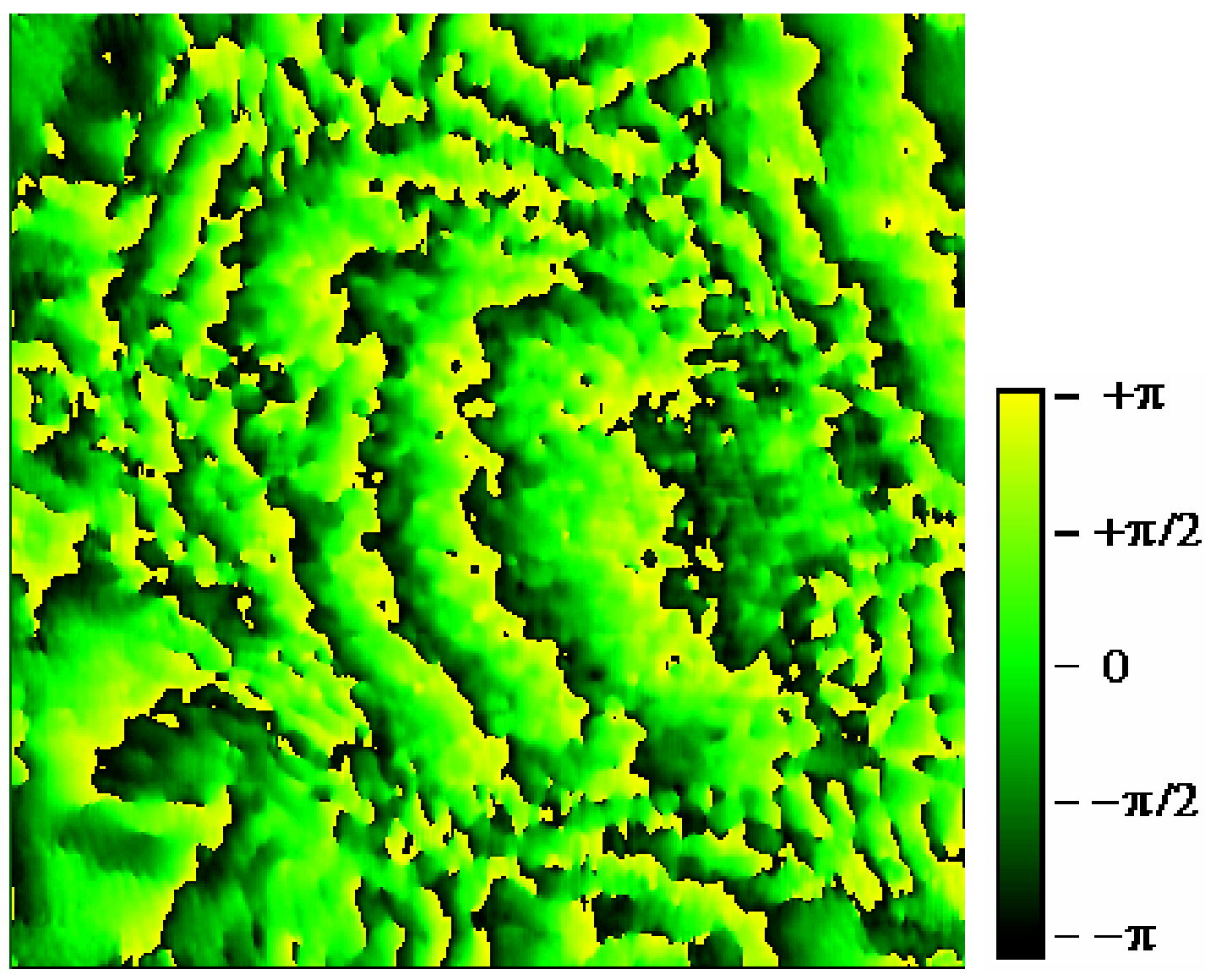

This phase image appears almost identical to the previous example, data set (a).

This is to be expected, as the sample was not physically moved, and all of the reconstruction parameters ( $x$ shift, $y$ shift, $z$ ) remained the same. This demonstrates the high repeatability of this technique. 
(c) Onion cells 1 - with aperture, OBJ $0.20 \mathrm{~V} / \mathrm{p}$, REF $0.27 \mathrm{~V} / \mathrm{p}$

Fig. 18, Amplitude Image (157 $\mu \mathrm{m}, 360$ pixels per side $)$ - Onion cell

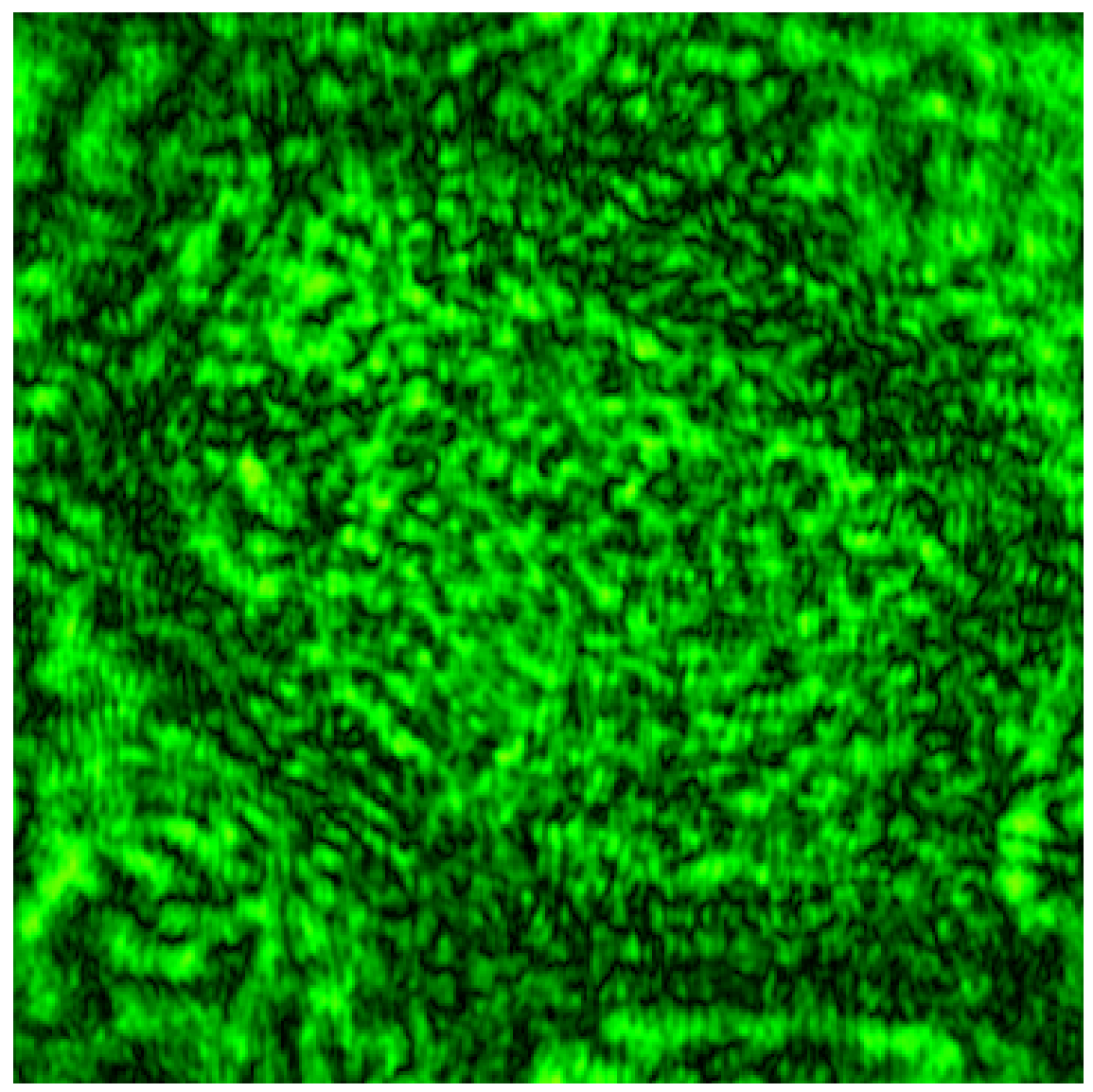

The overall light has been increased by about 30\% over the original data set (a). This amplitude image exhibits greater contrast between the light and dark areas than typically seen with lower light levels. The cell walls are clearly distinct from the main body of the cell. 
Fig. 19, Phase Image $(157 \mu \mathrm{m}, 360$ pixels per side $)$ - Onion cell

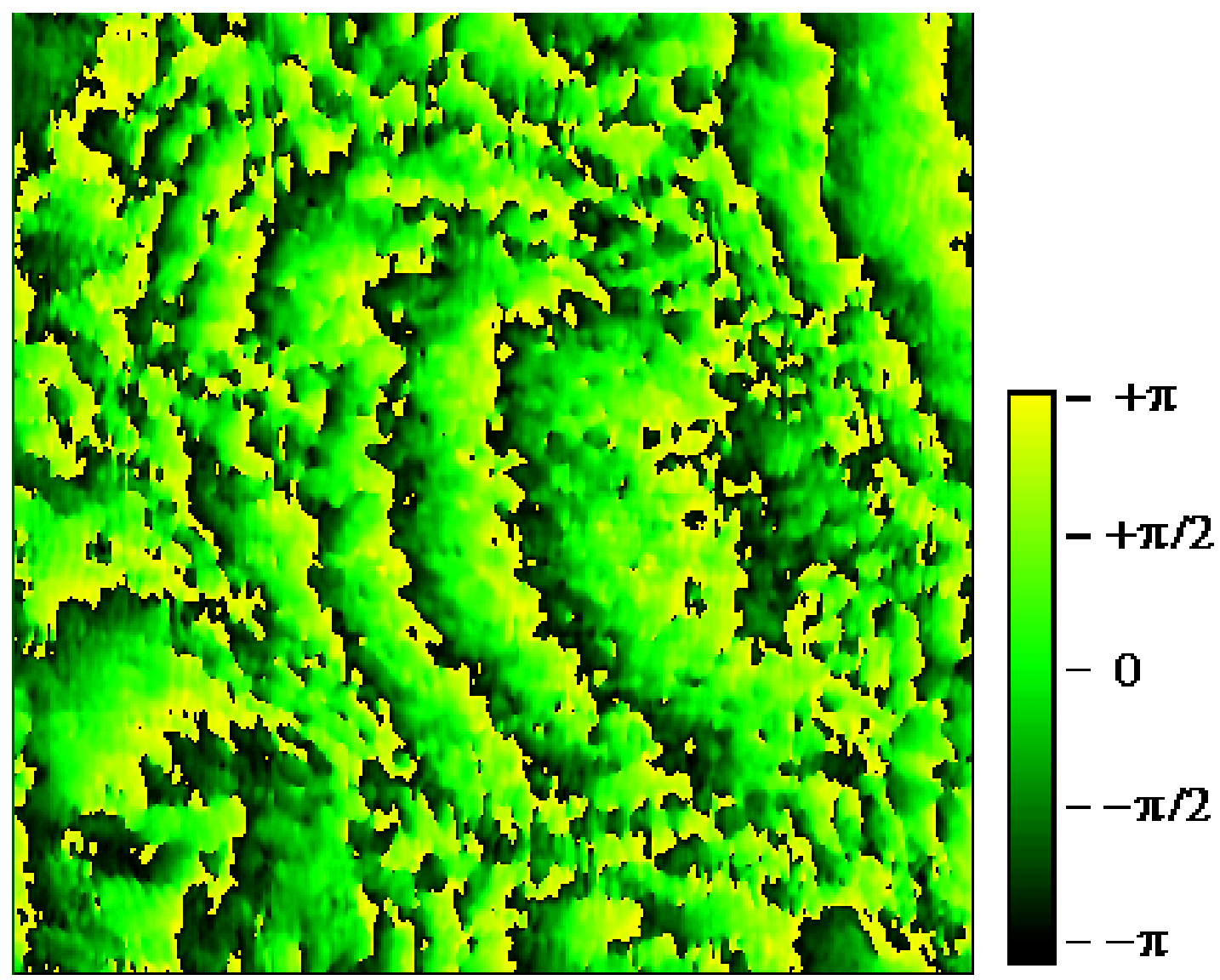

This phase image also looks basically identical to the previous 2 data sets, as expected. The phase between object and reference beams should not change, even if their amplitudes do, and we see that reflected here. The small vertical lines in the lower right portion of the image are a common noise feature. The increased light make this more pronounced as the light gets brighter. 
(d) Onion cells 1 - with aperture, OBJ $0.12 \mathrm{~V} / \mathrm{p}, R E F 0.23 \mathrm{~V} / \mathrm{p}, Z=\frac{1}{2} Z_{\text {min }}$

Fig. 20, Amplitude Image (157 $\mu \mathrm{m}, 360$ pixels per side $)$ - Onion cell

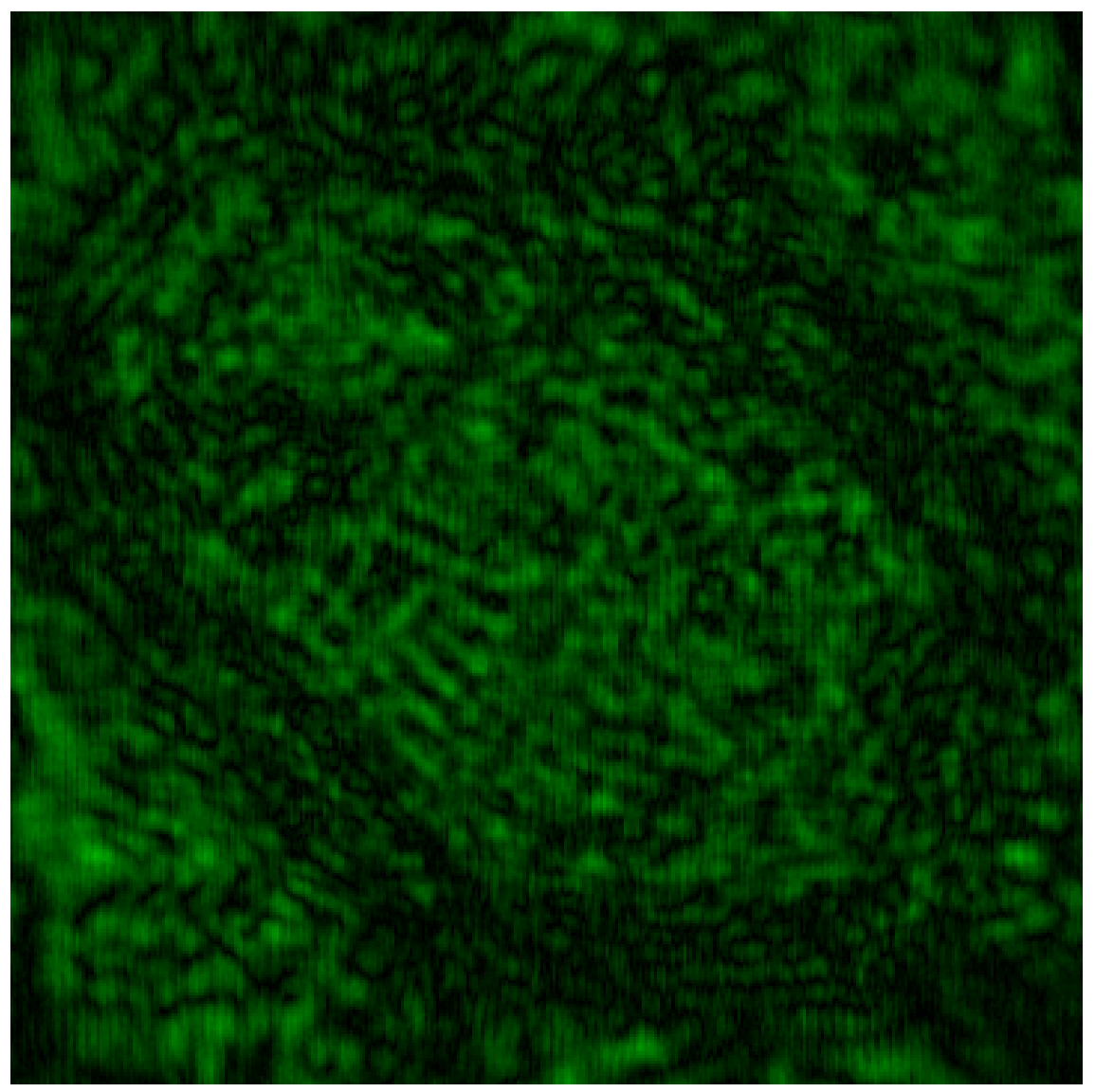

The overall light intensity and ratio between the object and reference beams has been returned to normal. The $Z$ distance has been reduced from $250 \mu \mathrm{m}$, (double the minimum distance) to $65 \mu \mathrm{m}$, about half the minimum distance. The cell body and walls are clearly discernable, even inside the limit set by the approximation conditions. This indicates that those conditions should be quite valid at farther distances. 
Fig. 21, Phase Image $(157 \mu \mathrm{m}, 360$ pixels per side $)$ - Onion cell

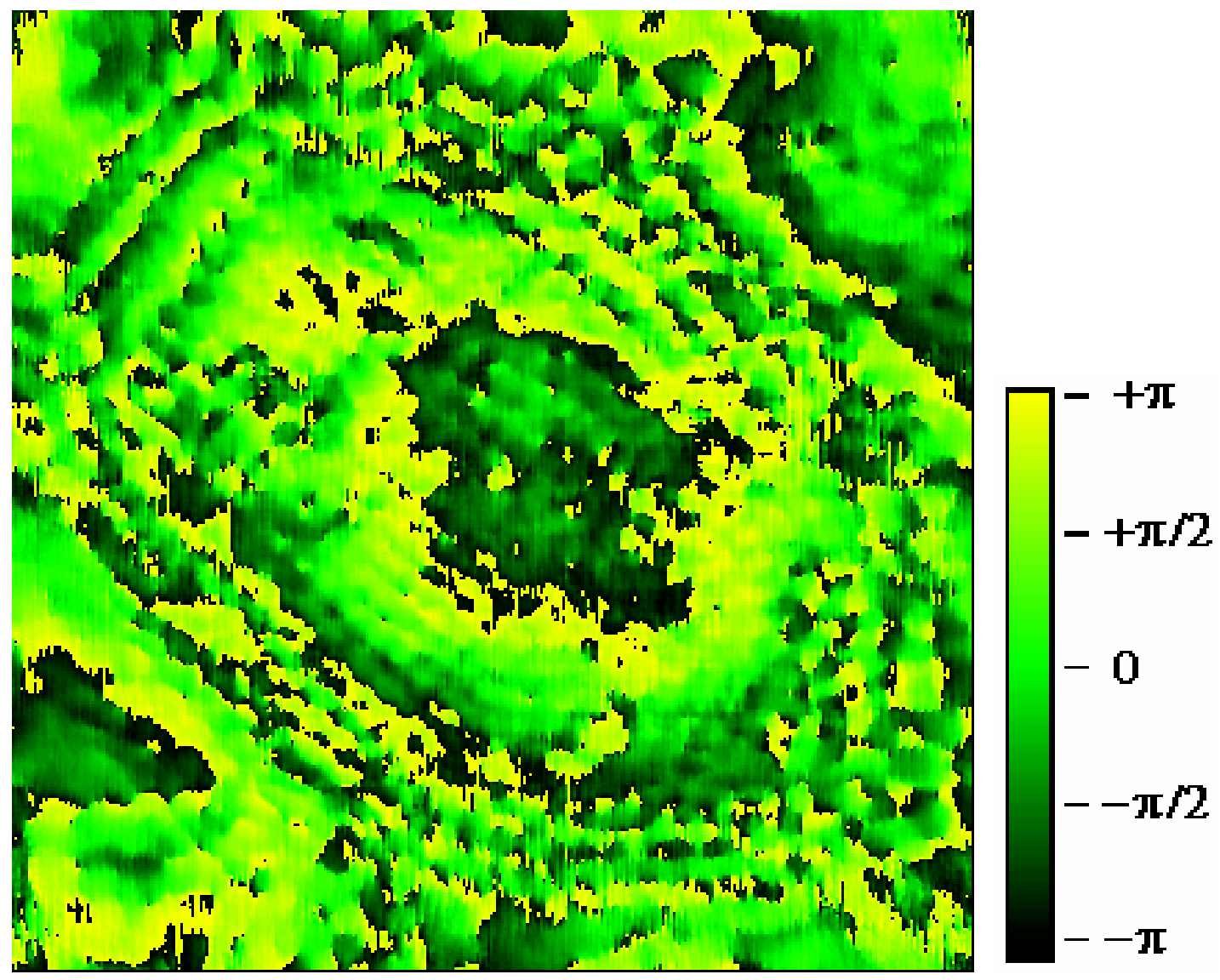

This phase image is markedly different from the first three data sets, which is to be expected since the sample itself was moved almost $100 \mu \mathrm{m}$. The noise lines throughout the phase image do offer an indication that the sample is perhaps to close to reconstruct the phase accurately. 


\section{High-magnification holography - onion cells 2}

The following data sets imaged a different portion of the same sample of onion cells. The first data set (a) was made without subtracting the $\mathrm{O}^{*} \mathrm{O}$ term. The image has vertical lines that are a common noise characteristic. The data set (b) immediately following is from the same initial hologram, but the $\mathrm{O}^{*} \mathrm{O}$ term has been subtracted. Most of the vertical lines have been eliminated, showing a smooth surface. The same $X$ shift, $Y$ shift, and $Z$ parameters were used for both sets of data.

Fig. 22, Direct Image (157 $\mu \mathrm{m}, 360$ pixels per side $)$ - Onion cells

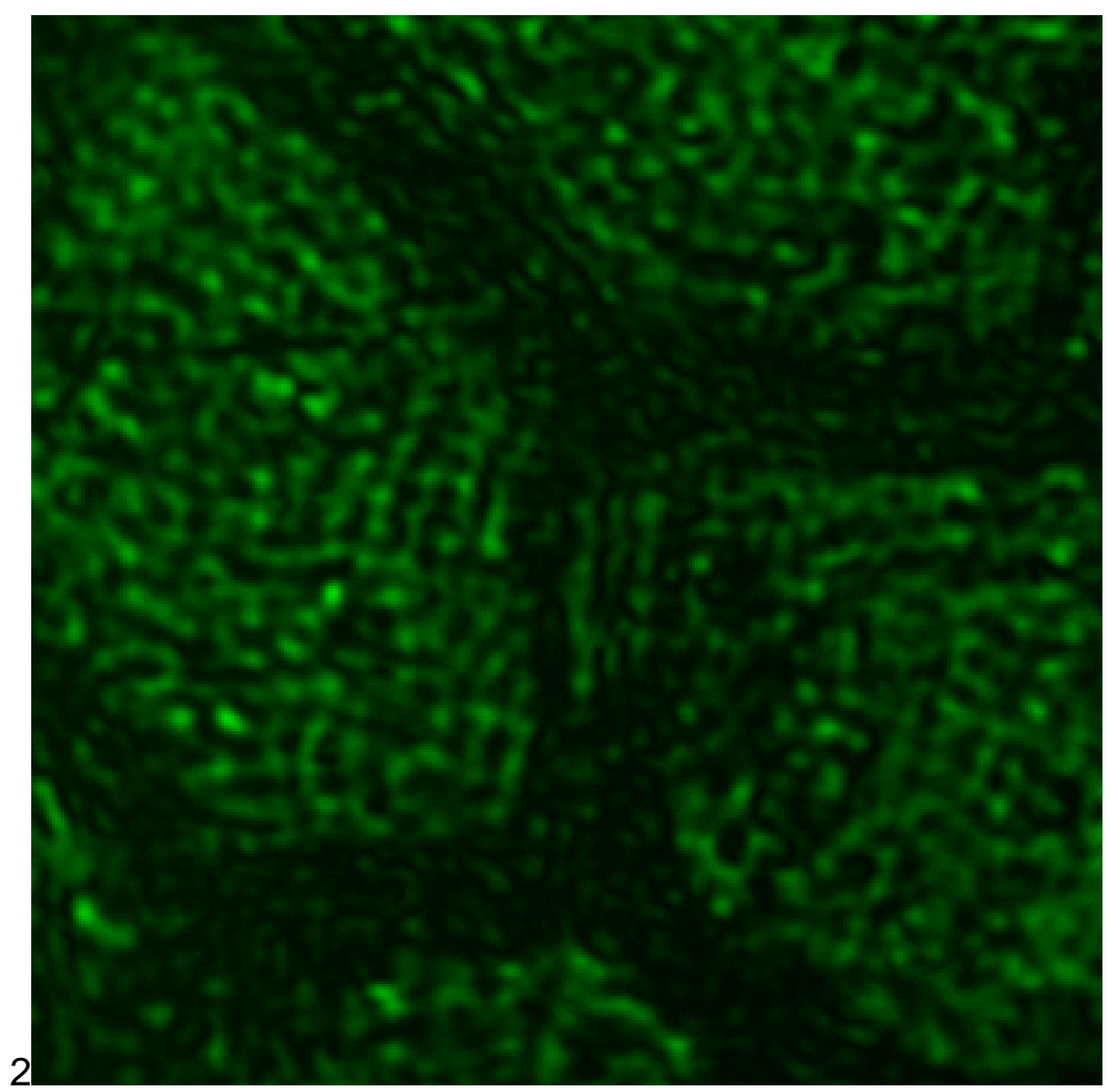


(a) Onion cells 2 - with aperture, $O^{*} O$ not subtracted

Fig. 23, Amplitude Image (157 $\mu \mathrm{m}, 360$ pixels per side $)$ - Onion cells

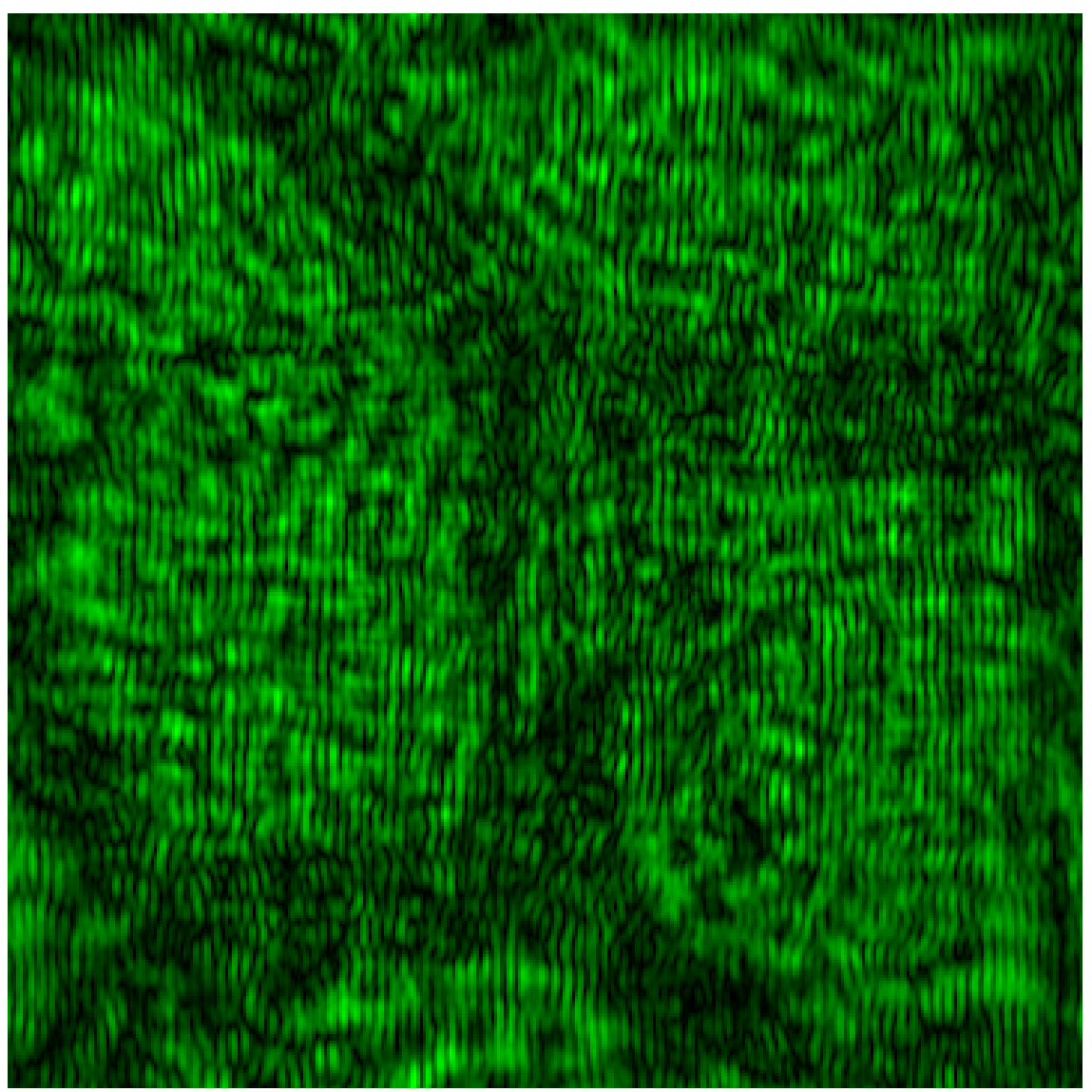

This amplitude reconstruction of the cells seen in Fig. 22 appears decent with a cursory inspection. However, the noise is so significant that it is visible in the amplitude image, not just the phase image. Obviously, subtracting off the object intensity improves image quality significantly. 
Fig. 24, Phase Image (157 $\mu \mathrm{m}, 360$ pixels per side $)$ - Onion cells

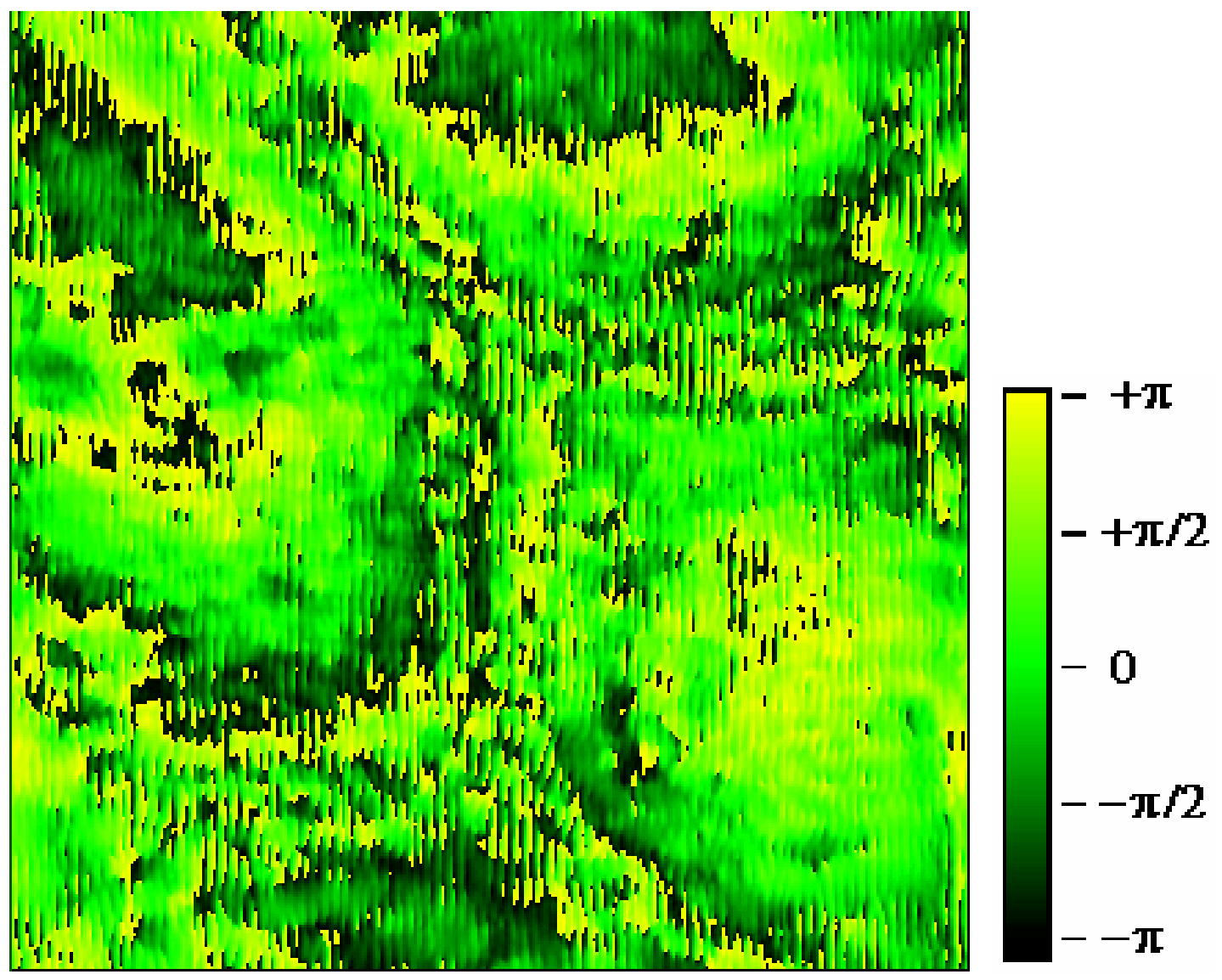

The noise is pronounced and evident over the whole image in this phase

reconstruction. While the cell walls and main bodies of the cells are visible, this image is not of very good quality. Most of the noise could be removed by subtracting the object beam from the data set, as done below. 
(b) Onion cells 2 - with aperture, $O^{\star} O$ has been subtracted

Fig. 25, Amplitude Image (157 $\mu \mathrm{m}, 360$ pixels per side $)$ - Onion cells

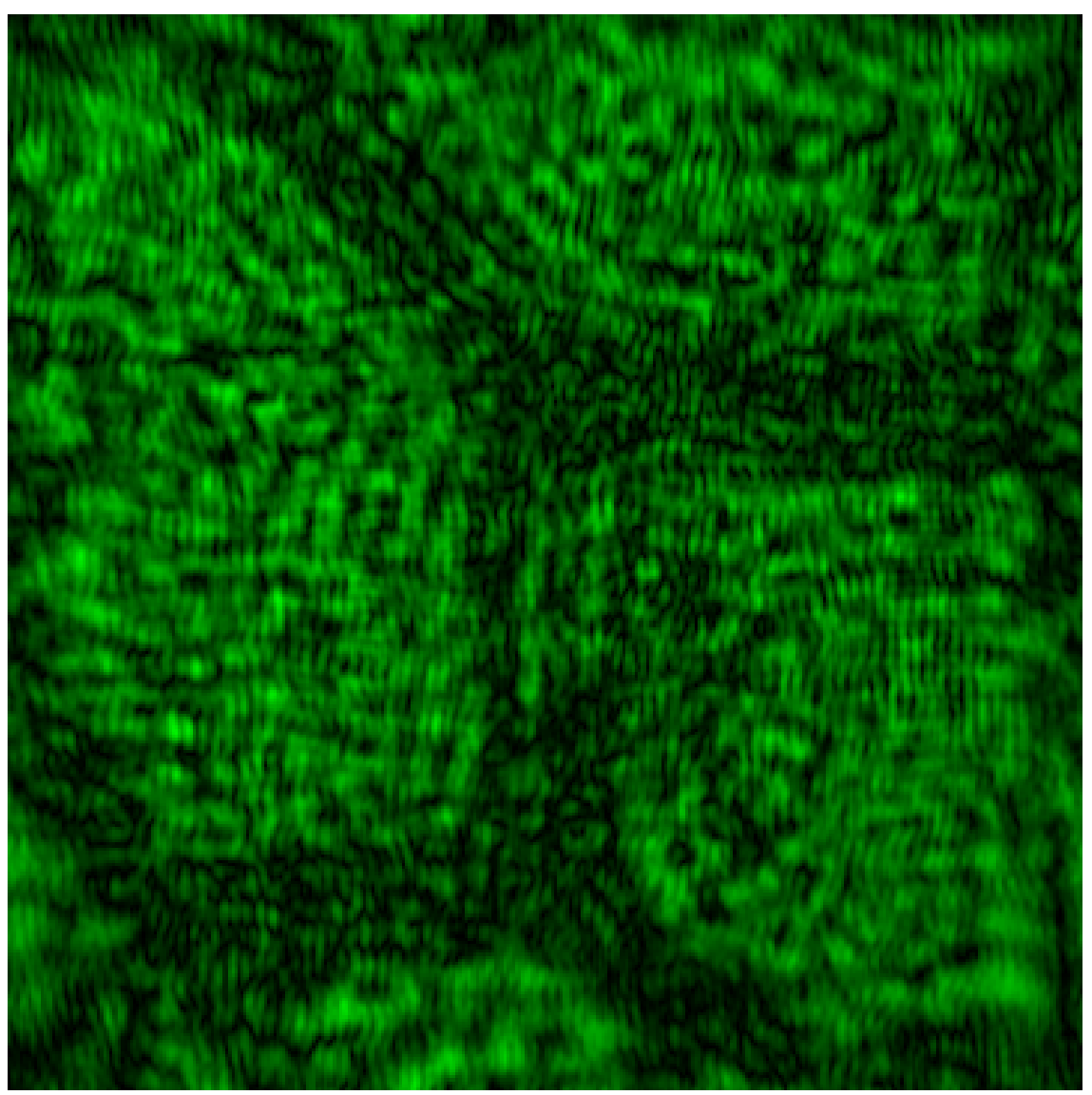

This amplitude reconstruction has been generated form the same hologram as the one acquired in data set (a), above, but now the object beam has been subtracted. This has almost entirely removed all of the noise from the amplitude reconstruction. 
Fig. 26, Phase Image $(157 \mu \mathrm{m}, 360$ pixels per side $)$ - Onion cells

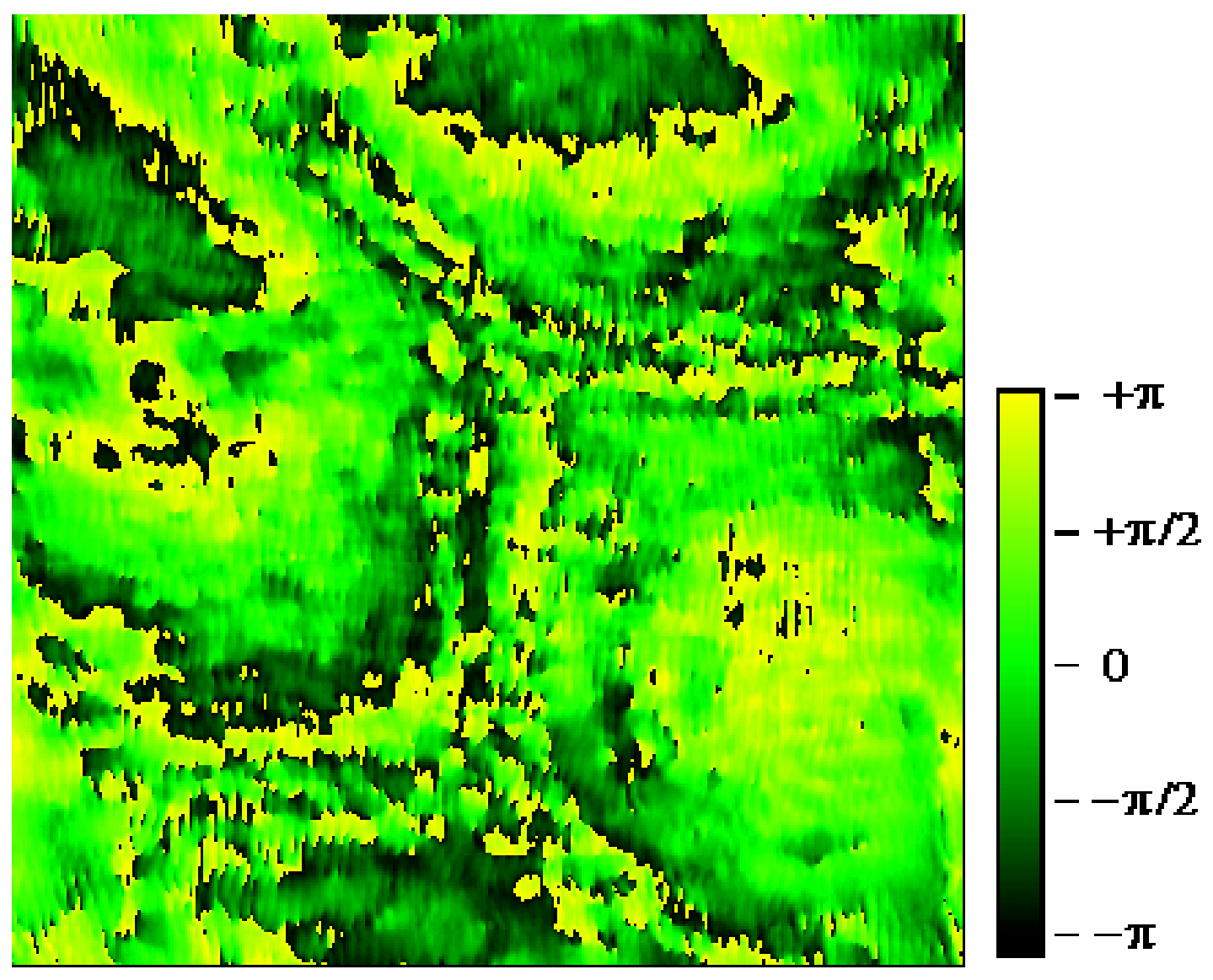

Much of the noise has been removed from the phase image now that the object beam has been subtracted. The two phase images (Fig. 24 and 26) generated form this hologram appear otherwise identical. Some noise remains, perhaps because it was several minutes before the object beam was finally subtracted, and (as mentioned above) the power output of the Nd:YAG 532 laser is prone to fluctuations. 
(c) Onion cells 2 - with aperture, OBJ .04 V/p, REF .07 V/p

Fig. 27, Amplitude Image (157 $\mu \mathrm{m}, 360$ pixels per side $)$ - Onion cells

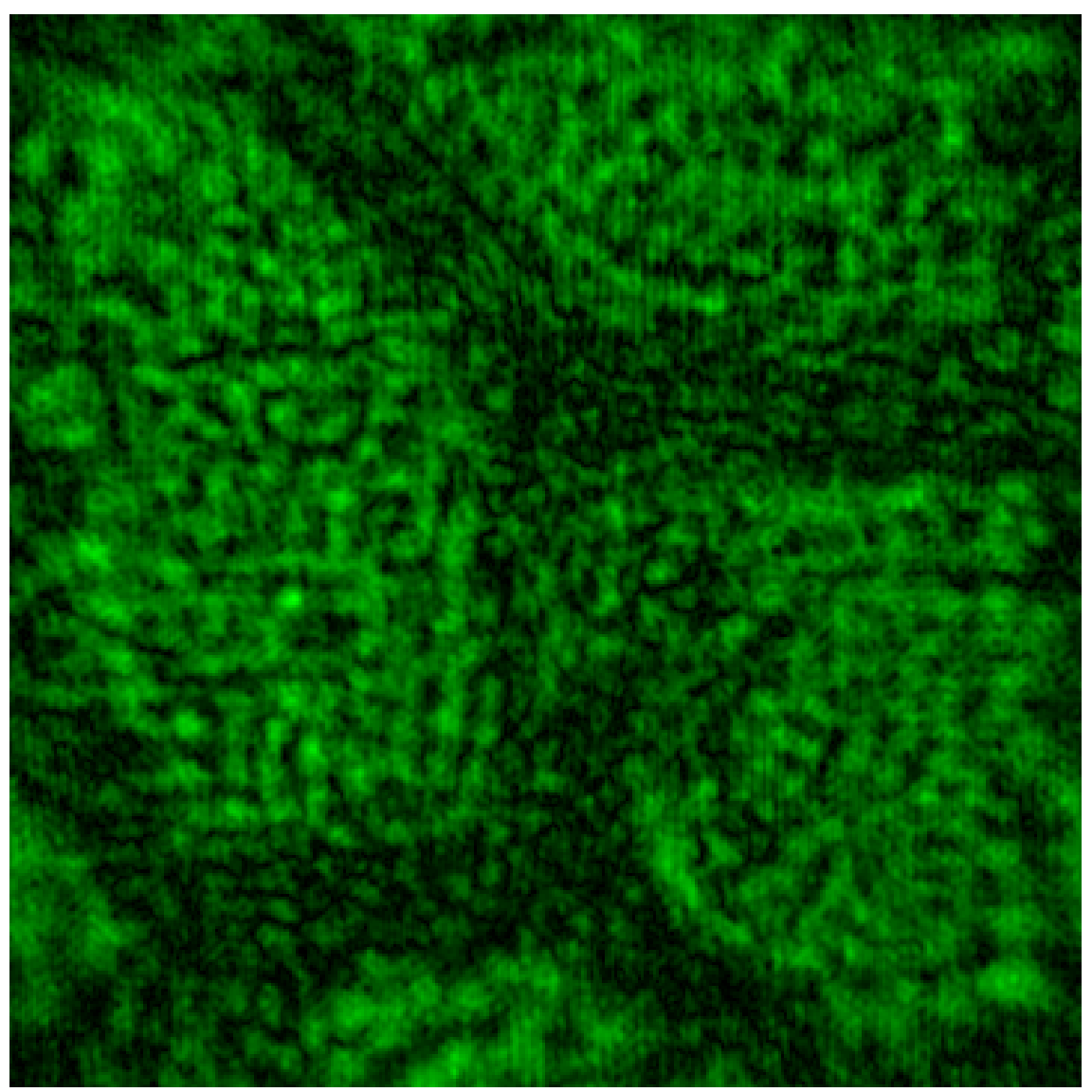

This data set was taken when the overall light had been reduced out of the linear region; the amplitude image is quite similar to the direct image. As in all of the images, the shadow of the aperture can be seen in the corners of the image. 
Fig. 28, Phase Image (157 $\mu \mathrm{m}, 360$ pixels per side $)$ - Onion cells

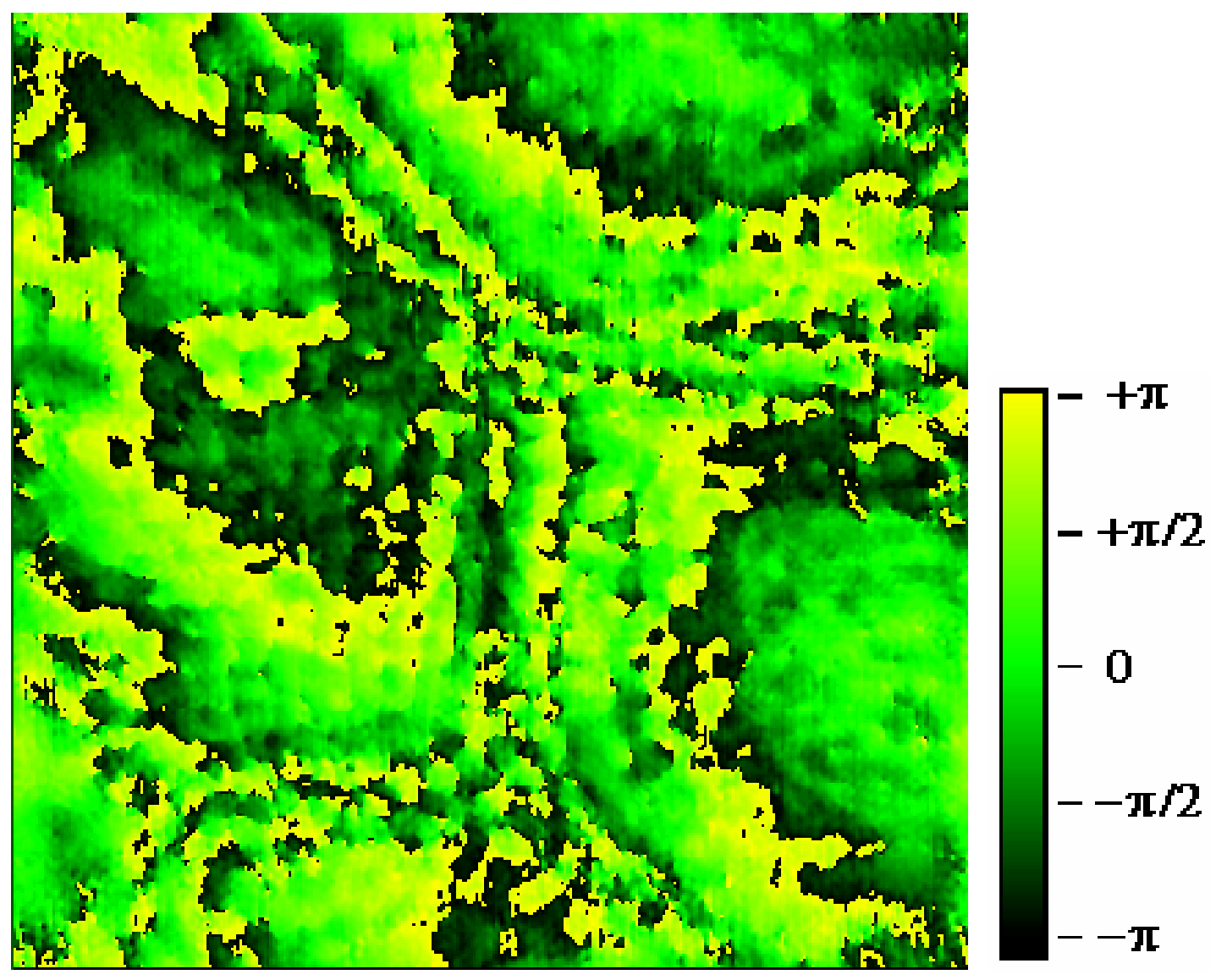

This is a fairly smooth phase image, but it does show some noise. The illumination was probably too far into the nonlinear region to generate accurate reconstructions. Nevertheless, the cell walls are distinct and visible, and some indication of the shriveling of the cell is visible in the cell on the left side of the image. 
C. Multiple-wavelength phase imaging holography

Multiple wavelength phase imaging digital holography has already been demonstrated in this laboratory [10]. Onion cells were used to test this technique with biological samples. These images were acquired using an achromatic, antireflection coated lens of focal length $1.5 \mathrm{~cm}(6.25 \mathrm{~mm}$ diameter $)$ and NA 0.2, Edmund Optics NT08-044. These results were presented at the BiOS conference in January 2004 (Kim, Parshall [17]).

While previous experiments had been run using solely the Nd:YAG 532 laser, it became necessary to introduce the HeNe 633 laser for these experiments to resolve the $2 \pi$ ambiguity. This was accomplished by the inclusion of a beamsplitter in the optical path. It was not necessary to replace any of the lenses in the system, since all lenses used were achromatic, in anticipation of this technique. The beamsplitter was carefully adjusted until both beams followed the same optical path. This was verified by visually examining the interference between the object and reference beams from each laser at the S' plane; they were found to be identical.

Once it was verified that the two beams were optically aligned, the data was collected in the manner described in the Procedures section. The experiment was first performed using a USAF Resolution Target, then later performed with onion cells. 


\section{Multiple wavelength holography - USAF resolution target}

The target was mounted on the translation stage, and brought to center on Group

4, Element 6 of the USAF target. The width of the lines from G4E6 is approximately $17 \mu \mathrm{m}$. The imaged area is approximately $157 \mu \mathrm{m}, 360$ pixels per side.

Fig. 29, Direct Image (157 $\mu \mathrm{m}, 360$ pixels per side) - USAF Target (HeNe 633)

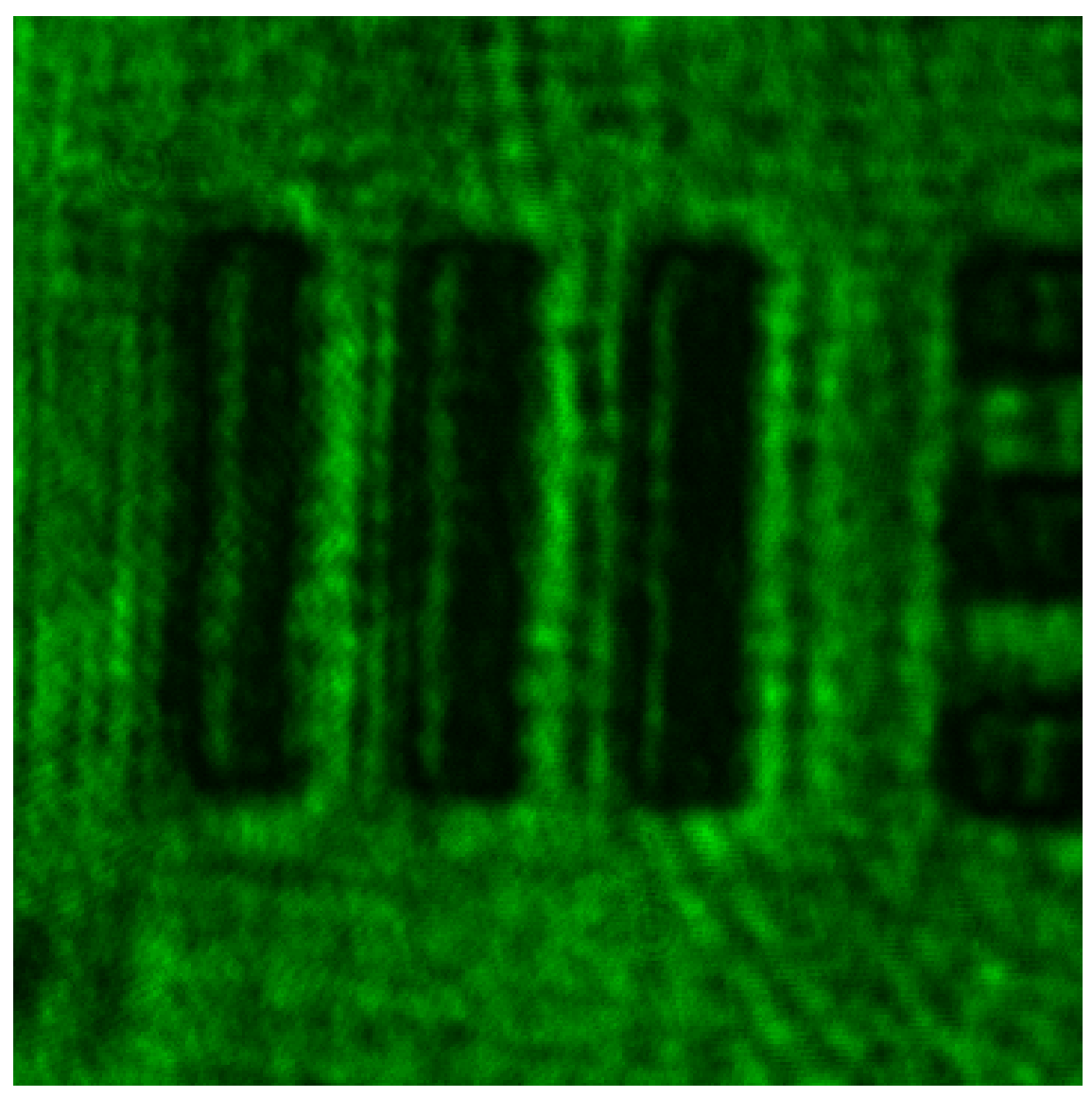


Fig. 30, Amplitude Image (157 $\mu \mathrm{m}, 360$ pixels per side) - USAF Target G4E6 (Nd:YAG532)

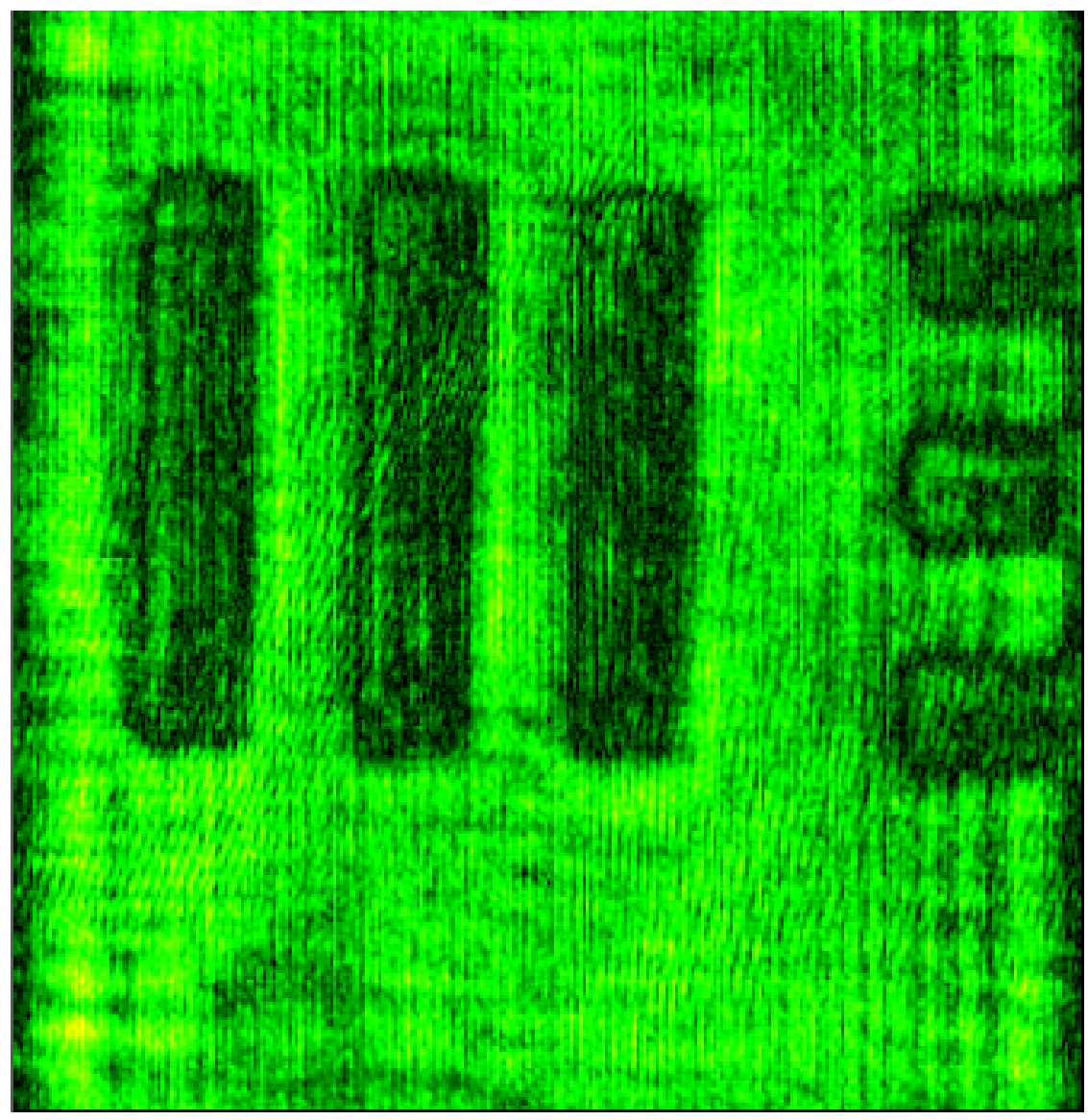

This amplitude reconstruction looks better than it has any right to. The lines are sharper than in the direct image, which is highly unusual. It is possible that the direct image was not quite in focus. The difference in phase from the surface of the chromium coating to the surface of the glass (visible in Fig. 31 and 32 below) implies a depth of about 1.14 radians for the $532 \mathrm{~nm}$ laser- around $100 \mathrm{~nm}$. 
Fig. 31, Phase Image $(157 \mu \mathrm{m}, 360$ pixels per side $)$ - USAF Target G4E6 (Nd:YAG 532)

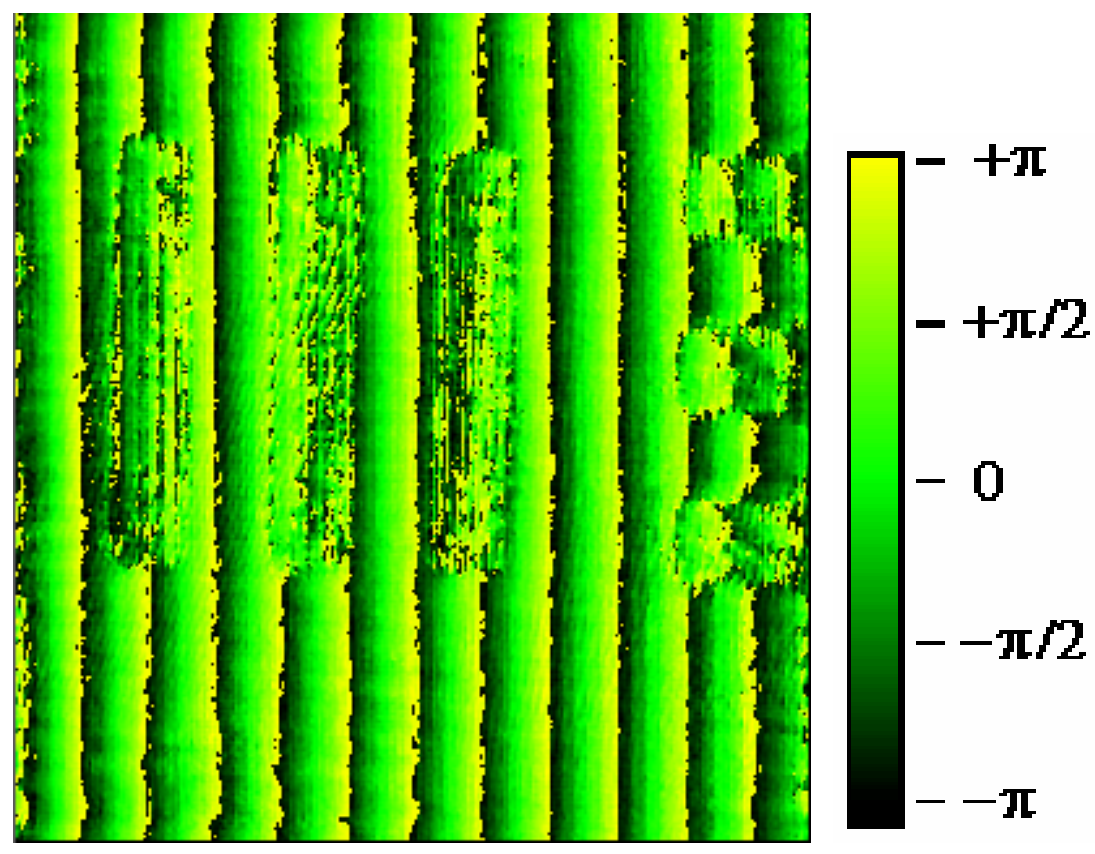

Fig. 32, Phase Image $(157 \mu \mathrm{m}, 360$ pixels per side) - USAF Target G4E6 (HeNe 633)

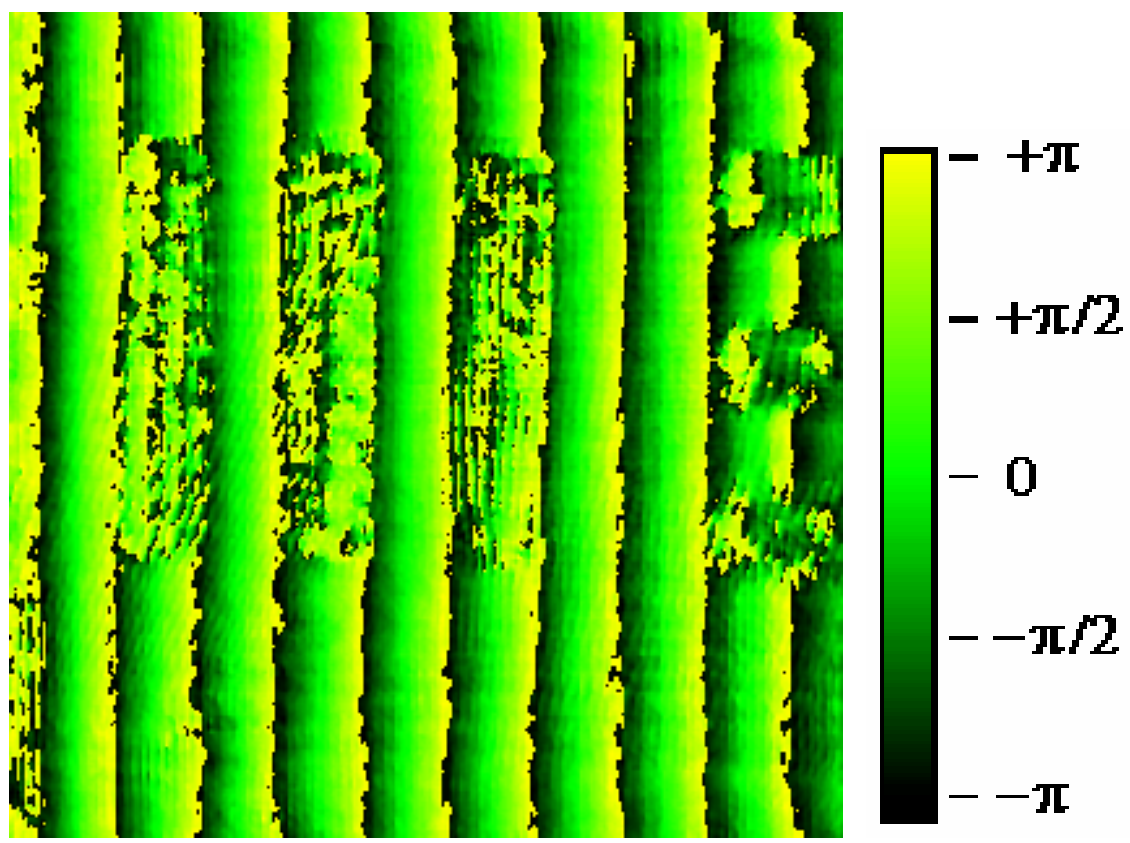


Fig. 33, Compound Image (157 $\mu \mathrm{m}, 360$ pixels per side $)$ - USAF Target G4E6 with $2 \pi$ ambiguities removed

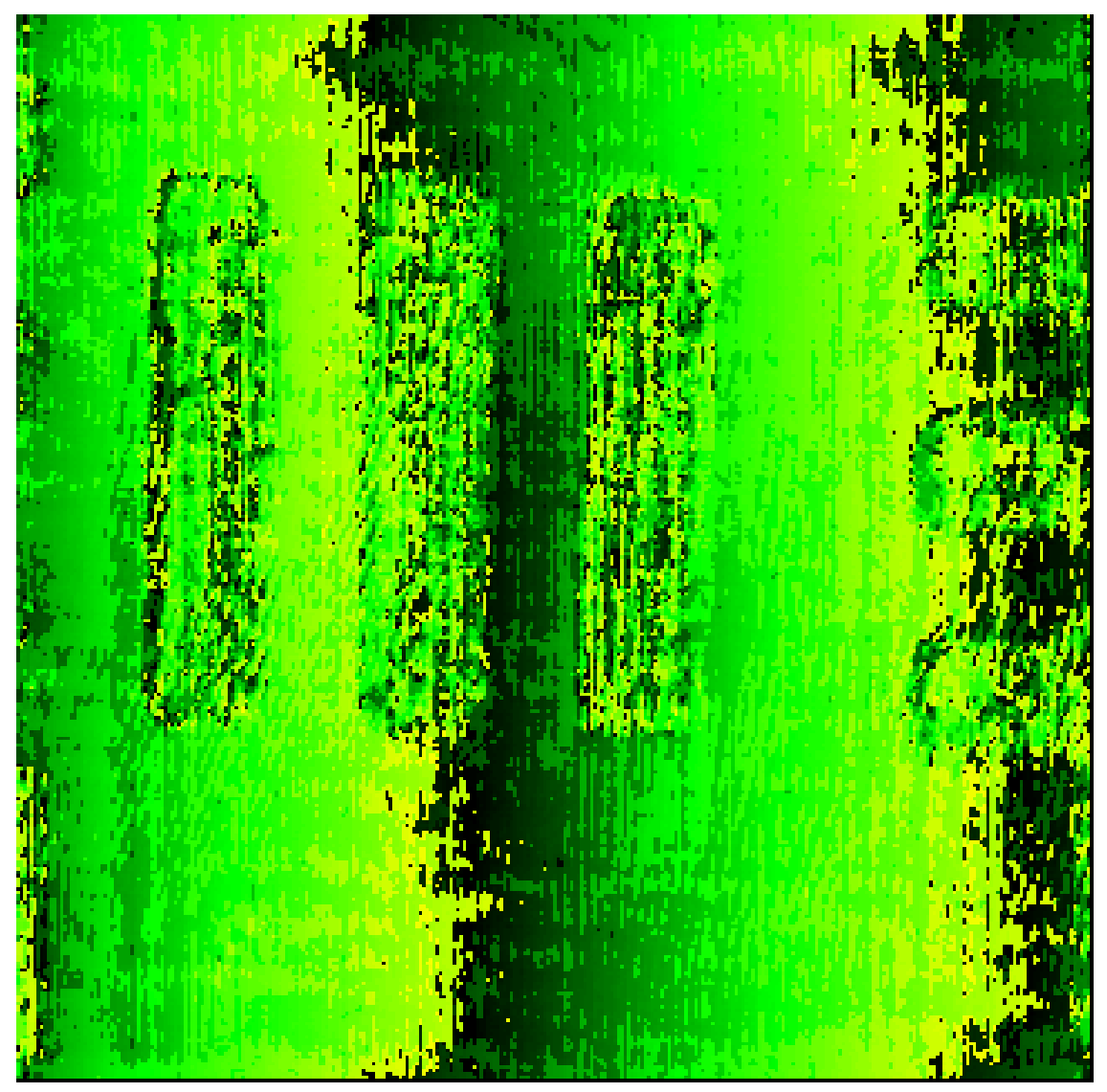

Most of the ambiguities have been removed. The image is still somewhat noisy, but not significantly more than the original phase images. 
Fig. 34, 3D Rotated Compound Image (157 $\mu \mathrm{m}, 360$ pixels per side $)$ - USAF Target G4E6 with $2 \pi$ ambiguities removed
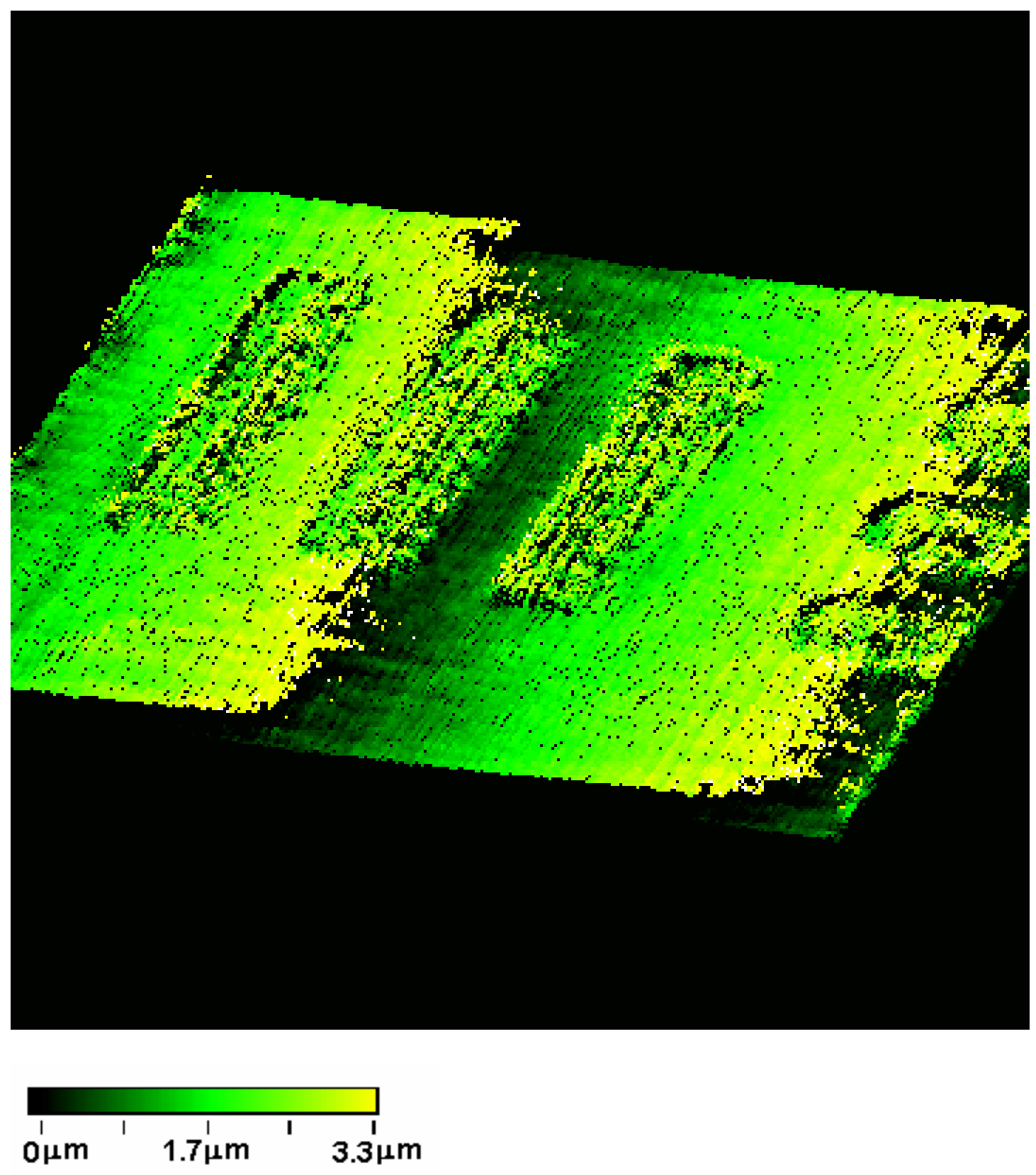

This is the compound image seen in Fig. 33, but it has been rotated to provide a better indication of the depth of the surface. 


\section{Multiple wavelength holography - onion cells 1}

The technique of MWPIH was next applied to onion cells. These images were $157 \mu \mathrm{m}, 360$ pixels per side. This set of data is fairly poor. It is included primarily to demonstrate the effectiveness of the MWPIH technique as applied to biological samples, even when the original data is of very low quality.

Fig. 35, Direct Image (157 $\mu \mathrm{m}, 360$ pixels per side) - Onion cells (Nd:YAG 532)

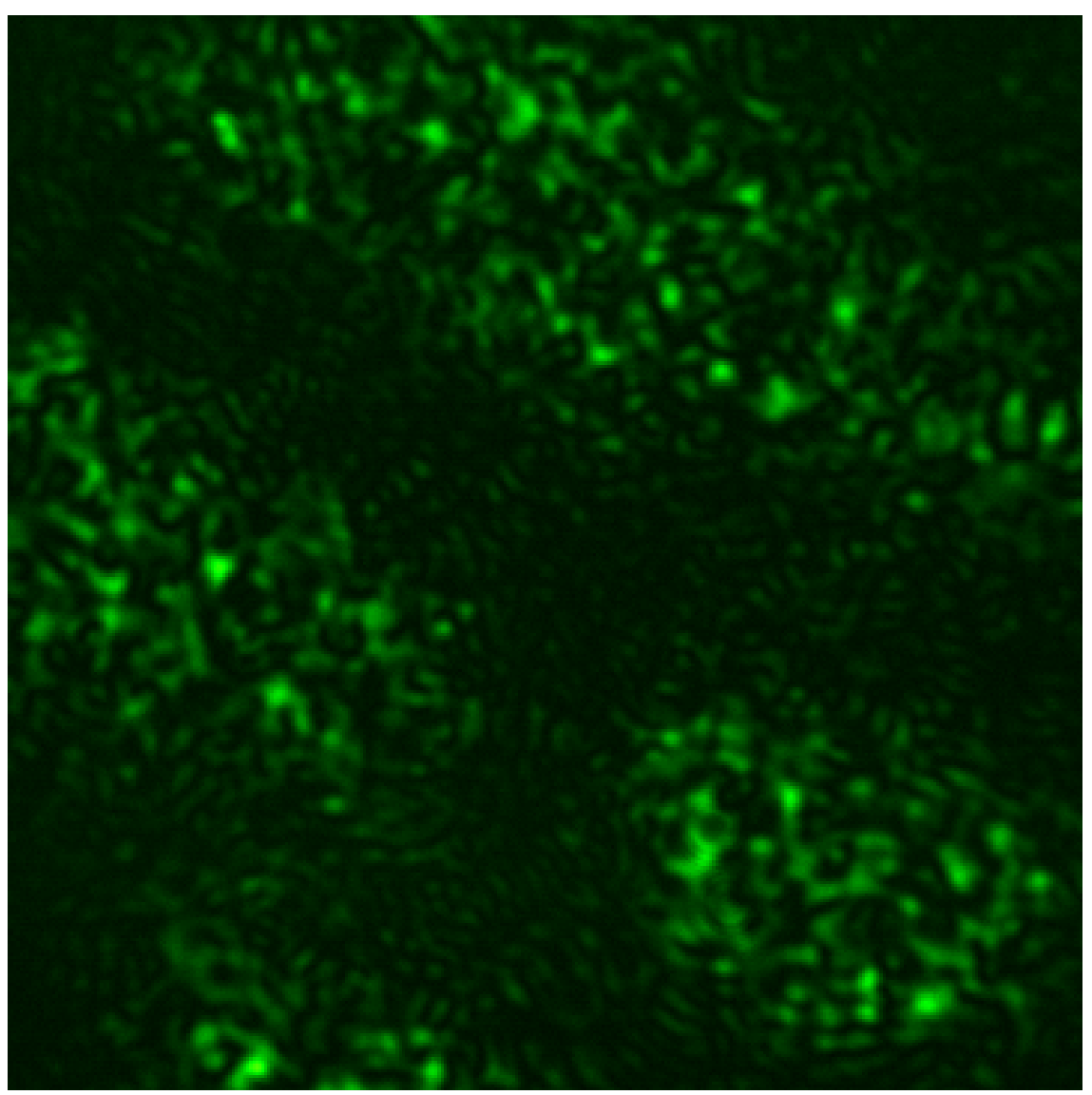


Fig. 36, Amplitude Image (157 $\mu \mathrm{m}, 360$ pixels per side) - Onion cells (Nd:YAG 532)

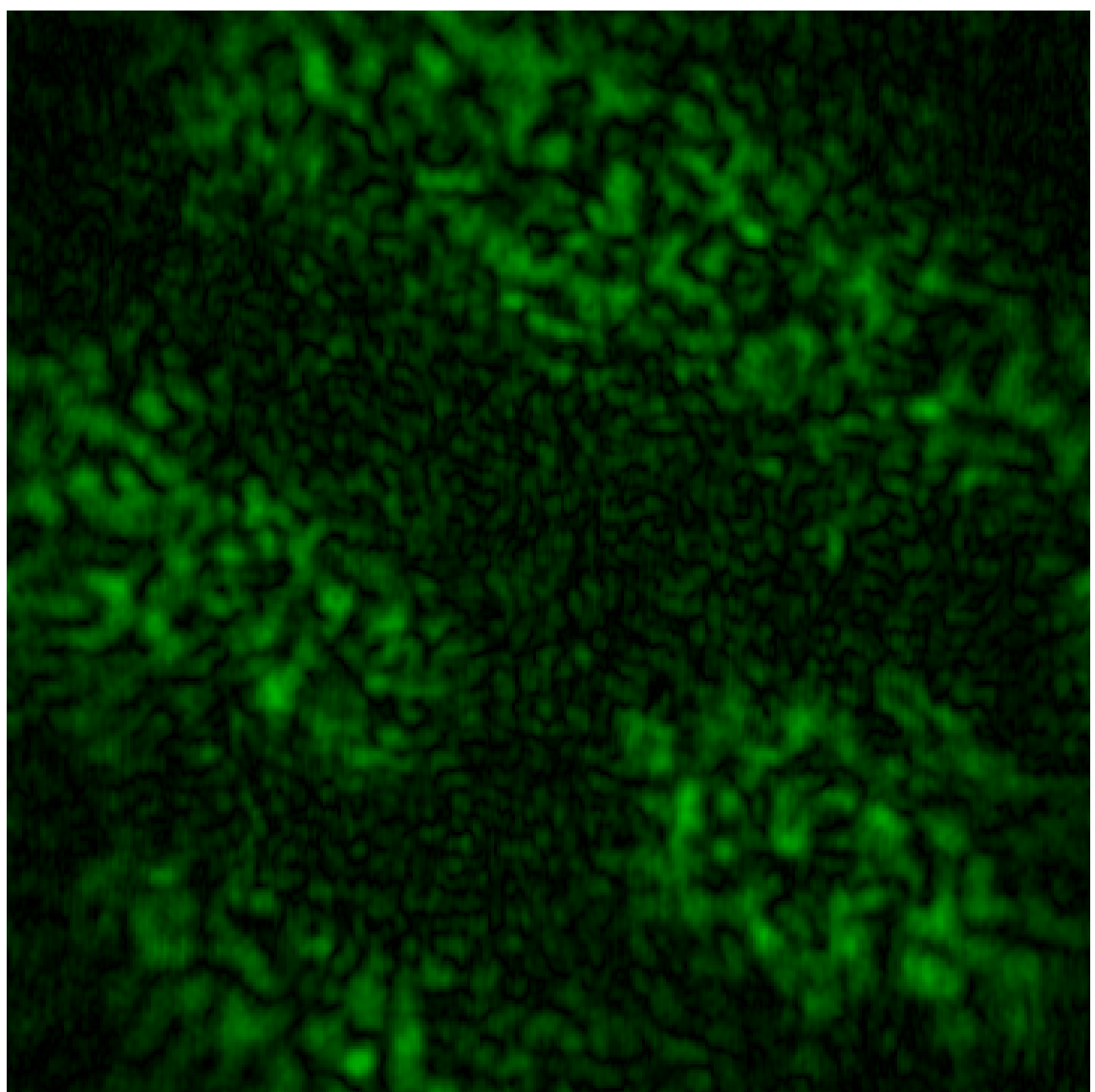

The direct and amplitude (Figs. 35 and 36 ) images primarily show blobs. This was a probably particularly poor sample of onion cells, yet even so some features can be made out in the phase images (Figs. 37 and 38) to the experienced eye. 
Fig. 37, Phase Image (157 $\mu \mathrm{m}, 360$ pixels per side) - Onion cells (Nd:YAG 532)

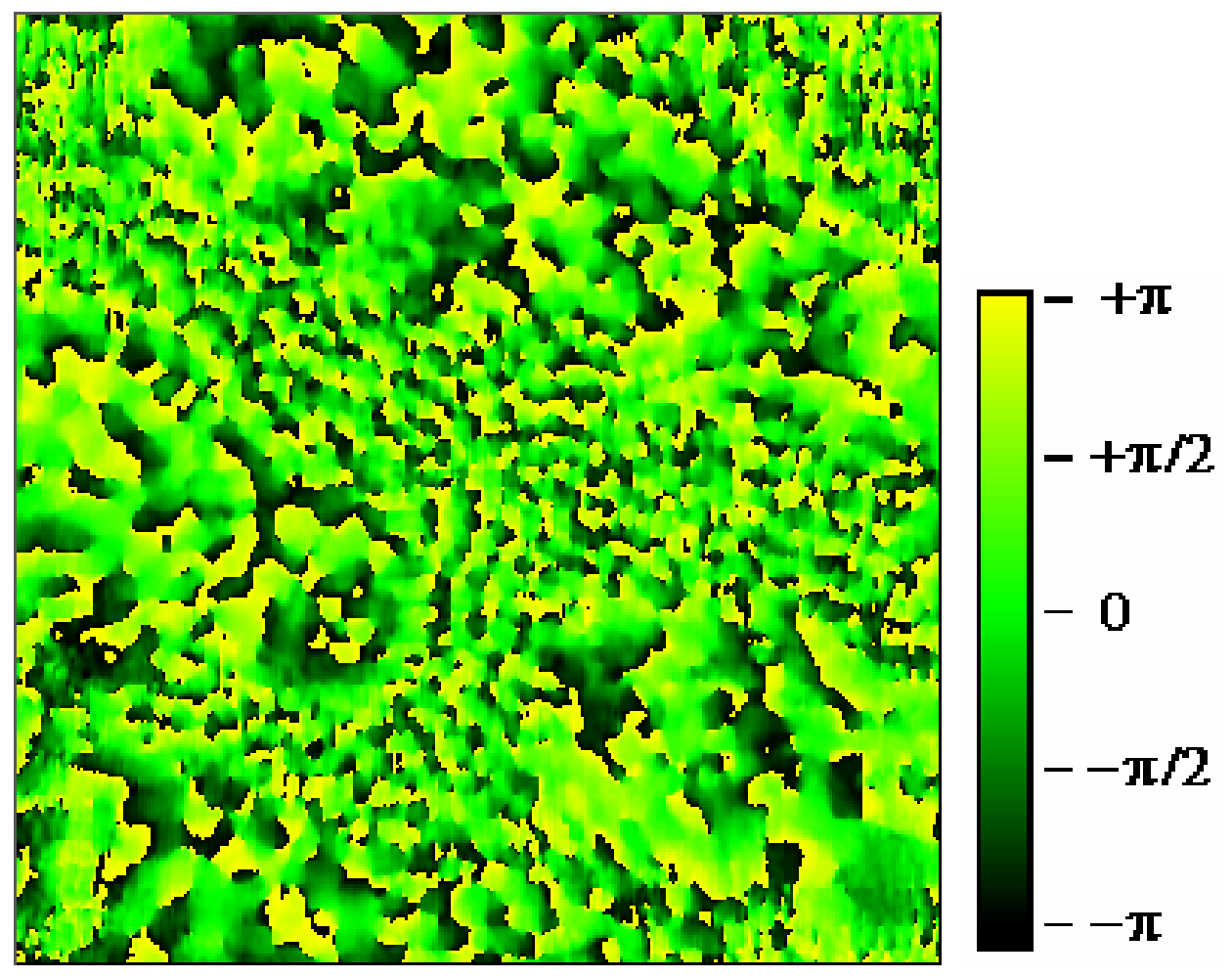

Fig. 38, Phase Image (157 $\mu \mathrm{m}, 360$ pixels per side) - Onion cells (HeNe 633)

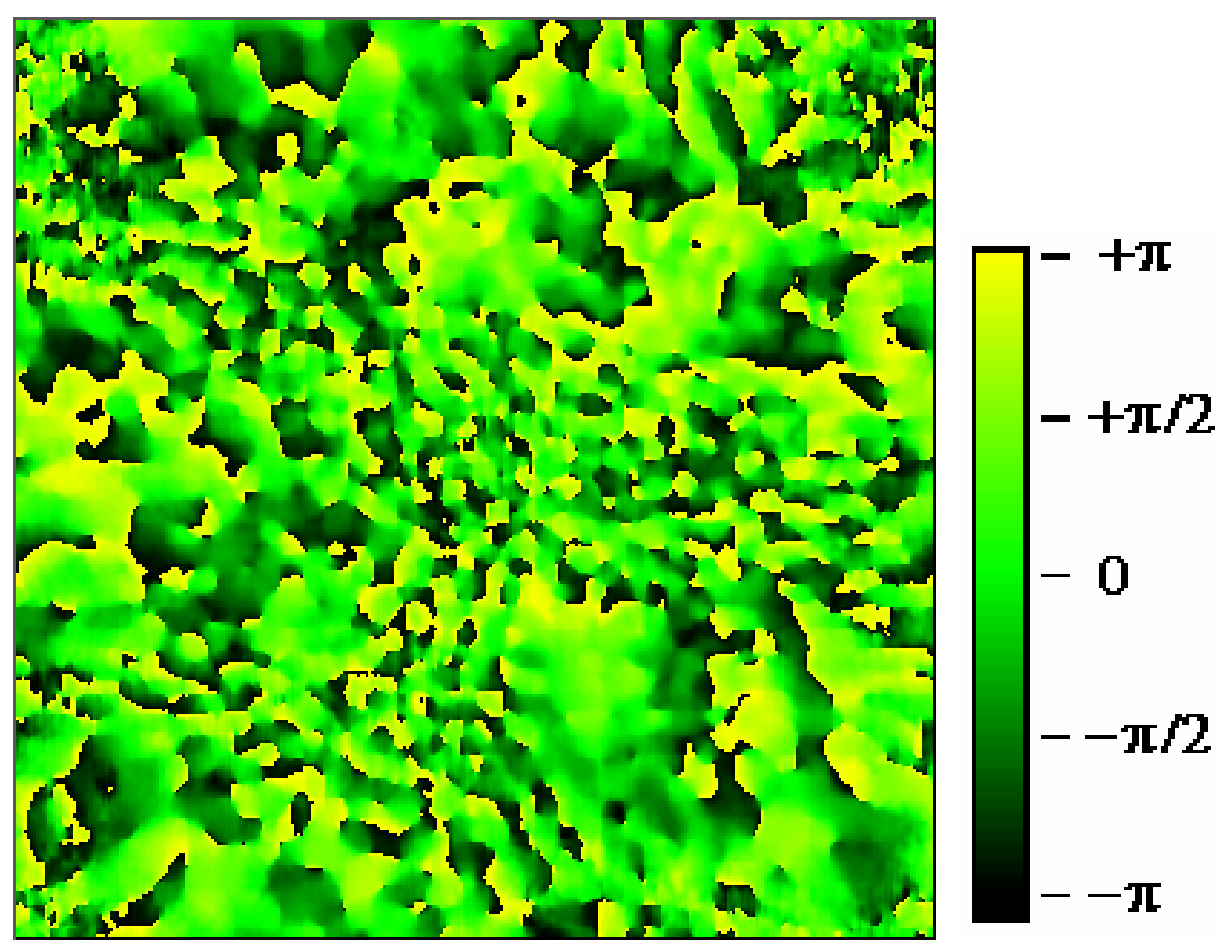


Fig. 39, Compound Image ( $157 \mu \mathrm{m}, 360$ pixels per side $)$ - Onion cells with $2 \pi$ ambiguities resolved (MWPIH)

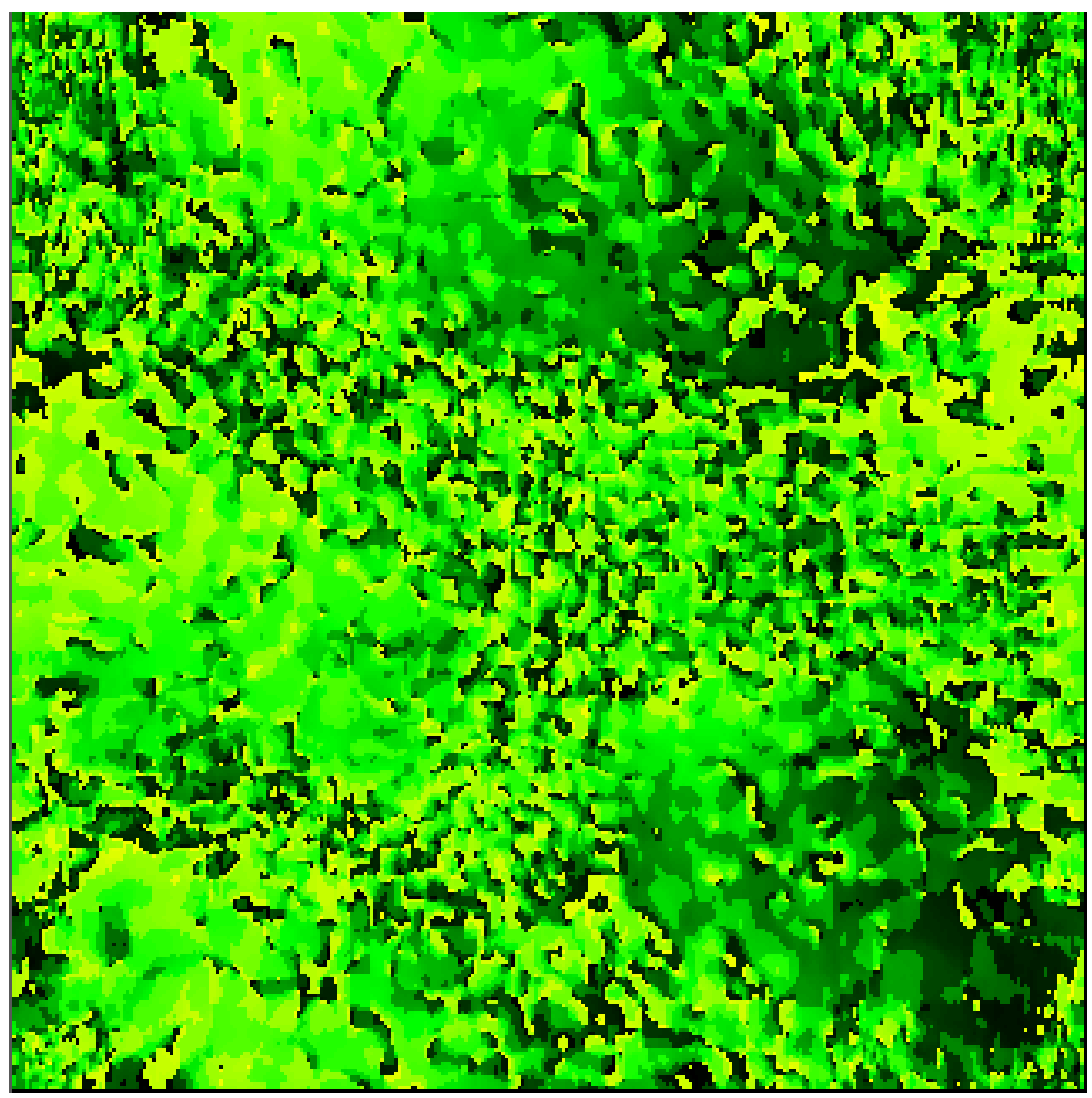

Most of the ambiguities have been removed, but there is still significant noise remaining form the original phase images. Nevertheless, the cells are fairly recognizable, especially with comparison to Fig. 35 . 
Fig. 40, 3D Rotated Compound Image (157 $\mu \mathrm{m}, 360$ pixels per side $)$ - Onion cells with $2 \pi$ ambiguities removed (MWPIH)
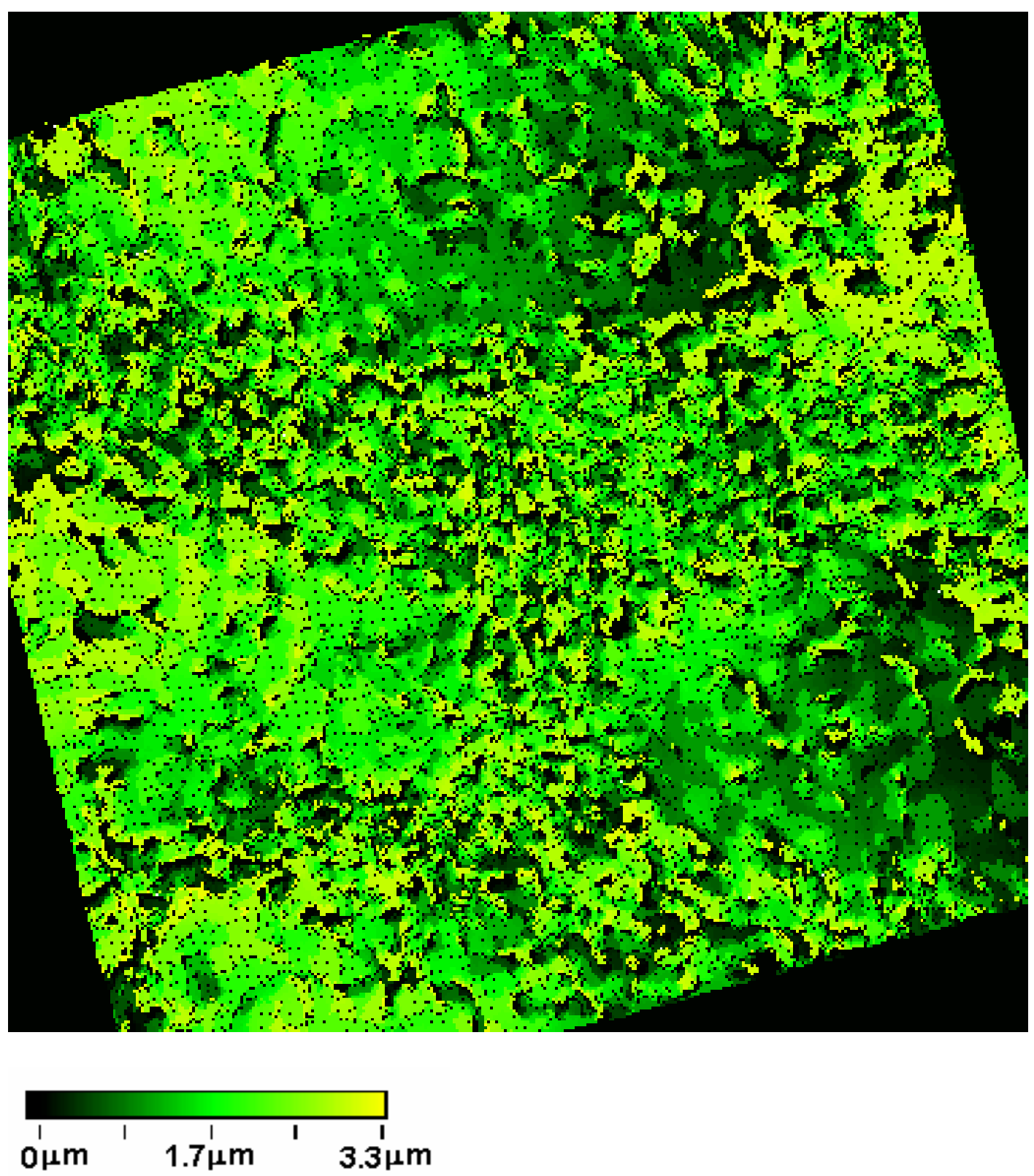

This 3D rotation of Fig. 39 shows the cells a little more clearly. 


\section{Multiple wavelength holography - onion cells}

This data set is fairly clean and smooth. The compound image is of fairly high quality. The images are squares $193 \mu \mathrm{m}, 472$ pixels per side.

Fig. 41, Direct Image (193 $\mu$ m, 472 pixels per side) - Onion cells (HeNe 633)

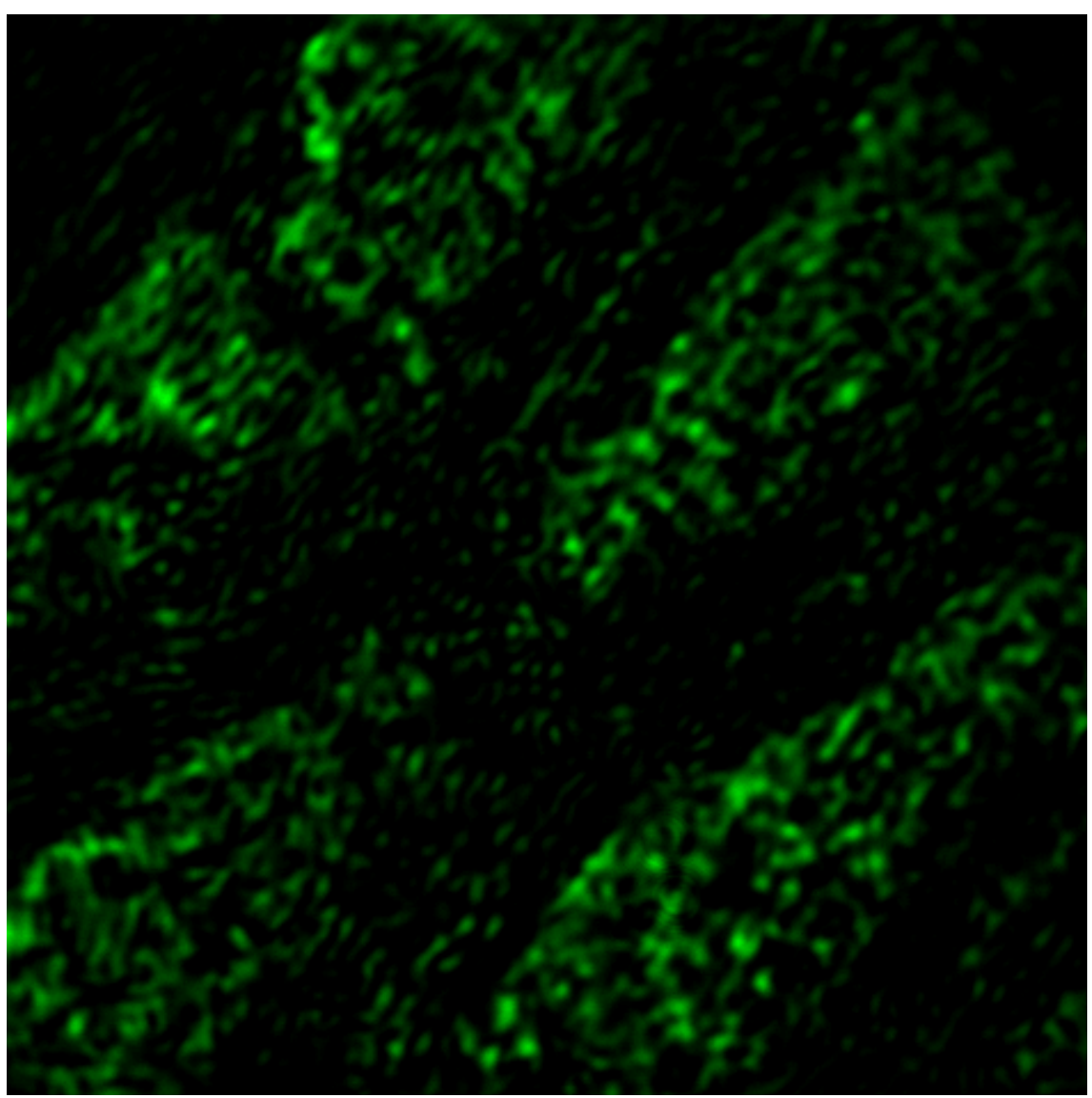


Fig. 42, Amplitude Image (193 $\mu$ m, 472 pixels per side) - Onion cells (Nd:YAG 532)

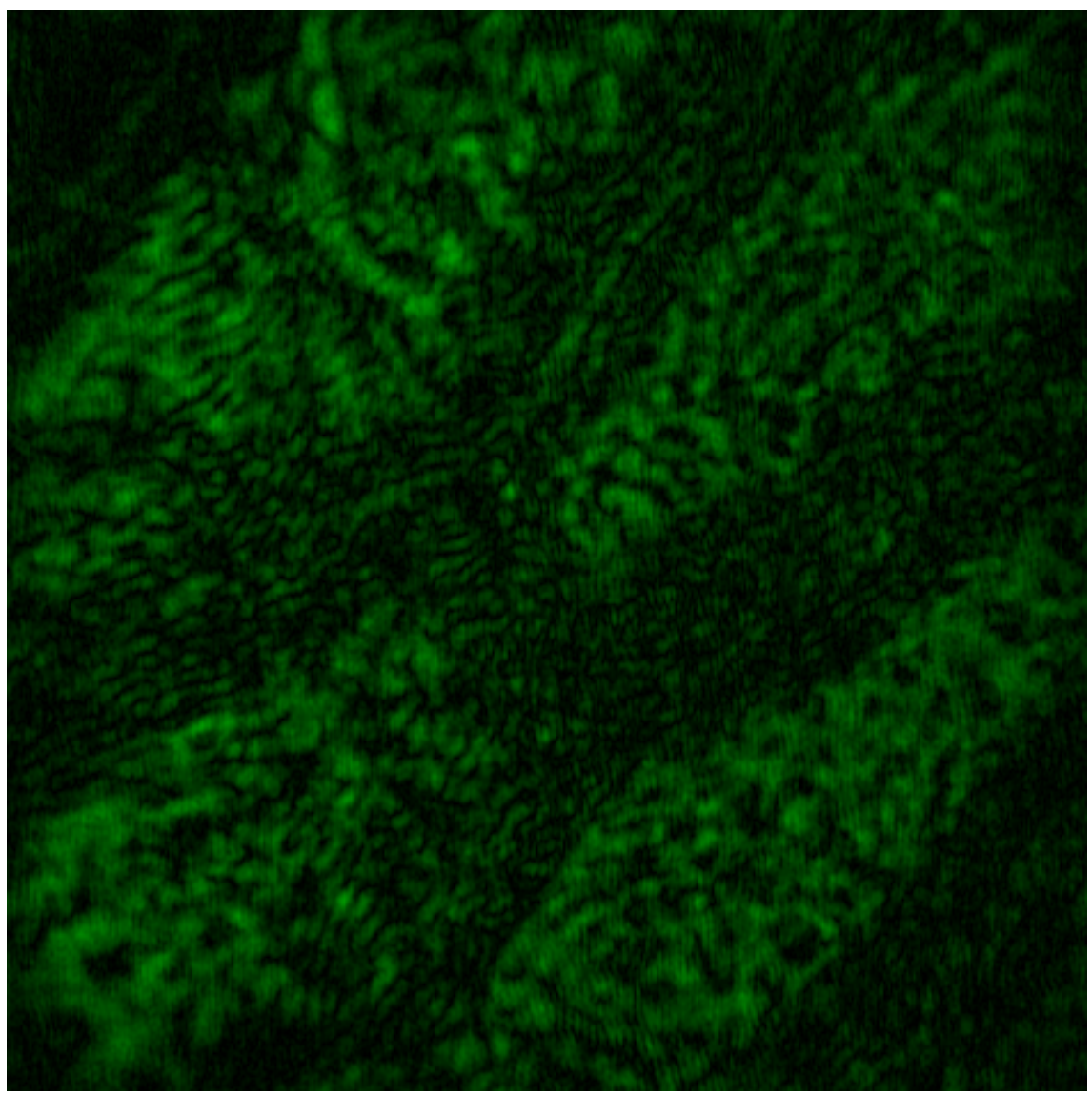

The amplitude reconstruction is very similar to the direct image. Portions of 4 cells are clearly visible in this sample. The phase images are clean and smooth, of particular interest is the phase reconstruction of the sample generated from the data gathered using the Nd:YAG 532. The surface of the cell on the upper right side of the image is clean and almost completely uniform; only at the very highest point of the cell is the $2 \pi$ ambiguity visible. While it is intuitively obvious that the cell continues to form a smooth surface, the MWPIH technique establishes this quantitatively. 
Fig. 43, Phase Image (193 $\mu \mathrm{m}, 472$ pixels per side) - Onion cells (Nd:YAG 532)

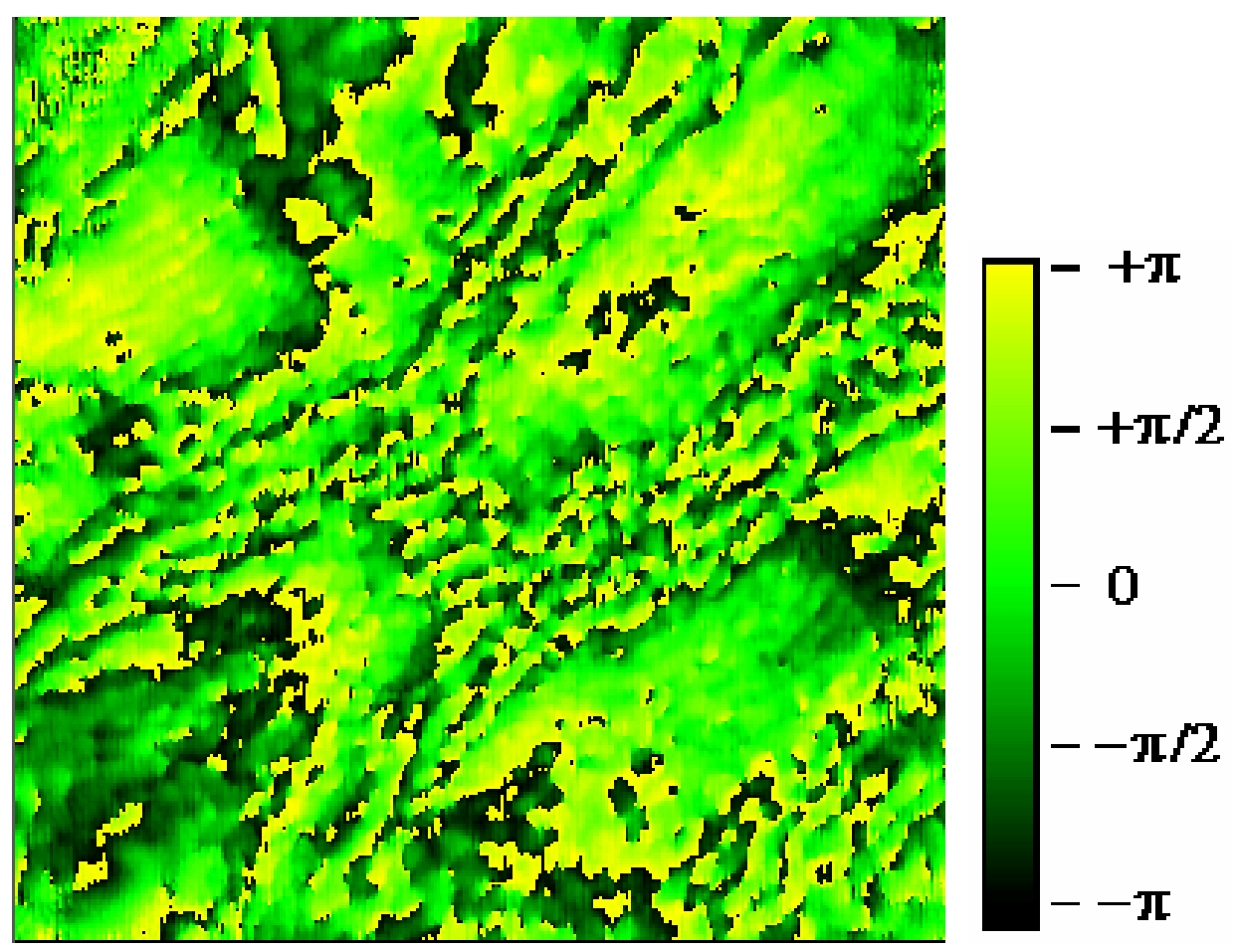

Fig. 44, Phase Image (193 $\mu$ m, 472 pixels per side) - Onion cells (HeNe 633)

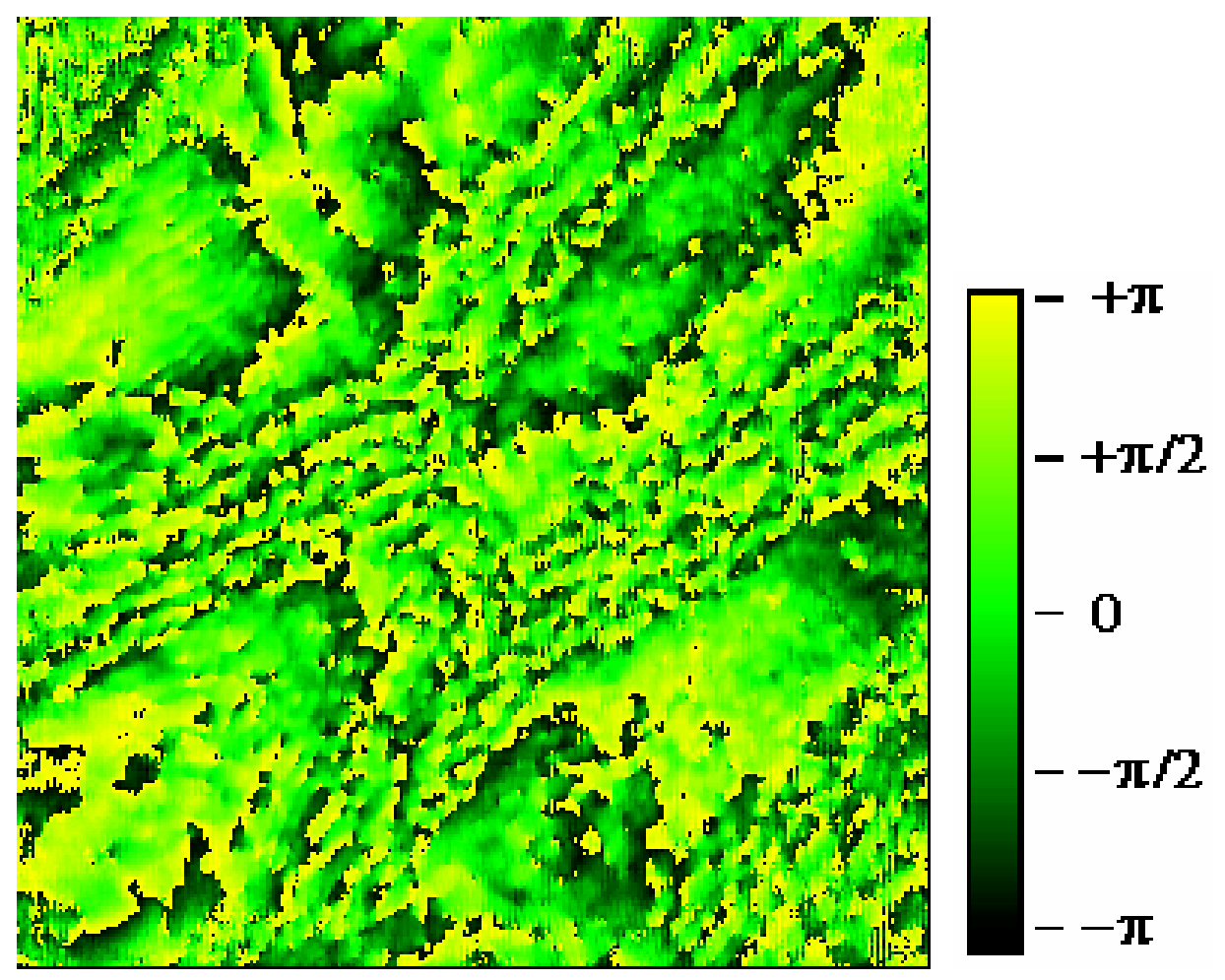


Fig. 45, Compound Image (193 $\mu \mathrm{m}, 472$ pixels per side $)$ - Onion cells with $2 \pi$ ambiguities removed (MWPIH)

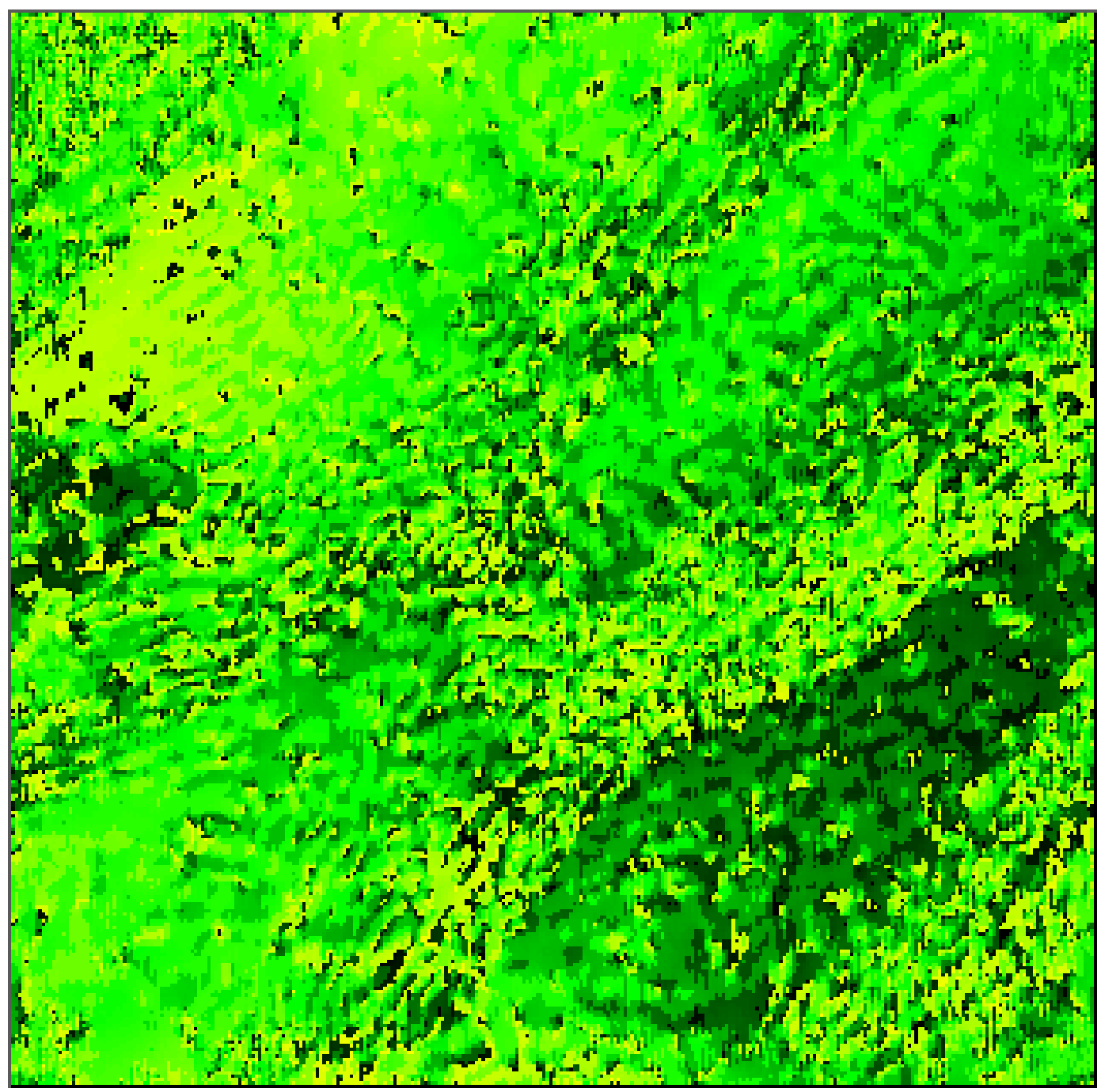

The 4 cells have been clearly resolved and all of the ambiguities removed in this image. The surfaces of the cells are fairly smooth and quite distinct form the cell walls. 
Fig. 46, 3D Rotated Compound Image (193 $\mu \mathrm{m}, 472$ pixels per side $)$ - Onion cells with $2 \pi$ ambiguities removed (MWPIH)
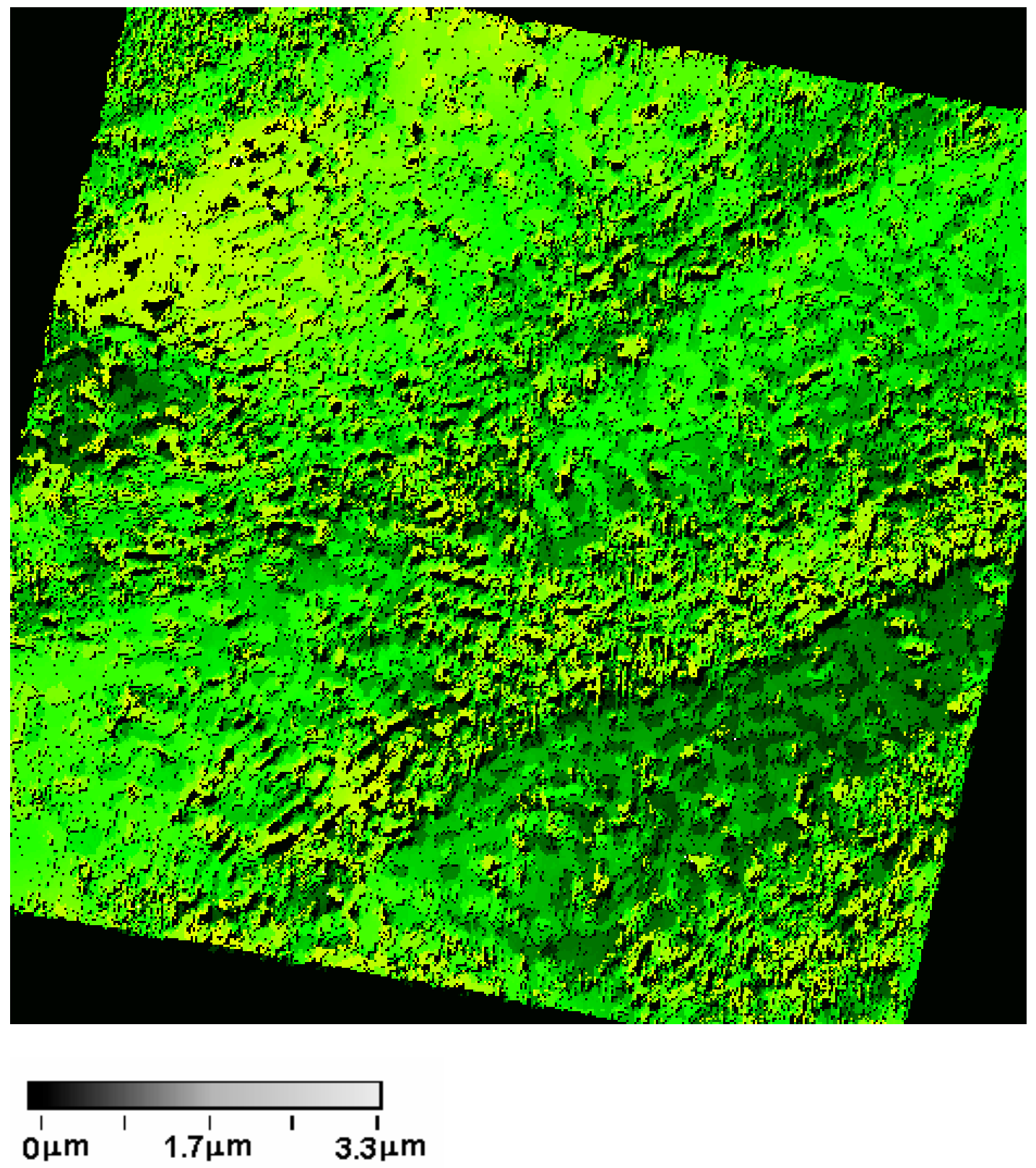

This is the same compound image as Fig. 45 , but it has been rotated to allow for a different view of the cells. 


\section{High-resolution biological imaging}

Having established the validity of the system for biological samples using the MWPIH technique [17], it was decided to try to increase the magnification and resolution of the system. This was accomplished by replacing L3 with an achromatic 20X DIN microscope objective lens with effective focal length 8.55 $\mathrm{mm}$ and a NA of 0.4 , Edmund Optics 38339 . While this lens was allegedly antireflection coated, the reflection coming from it was significantly greater than the reflection observed with the lens used for the low resolution experiments.

Because of the increased back reflection, it was necessary to shift and tilt the lens off of the optical axis. This introduced an astigmatism ${ }^{14}$ that plagued images acquired while this lens was in place. In particular, when the target was moved away from the S plane to the I plane, the image shifted, since the object could no longer be moved along a single optical axis. However, the magnification was so high that only a small translation was required to satisfy the diffraction approximation conditions. Many of the images acquired with this lens are of lower reconstruction quality (relative to the direct image quality) due partly to this effect. For example, some reconstructions suffer from "wraparound" effects; portions of the image that should be on the left have been reproduced on the right, etc. Finally, some images in the printed version were adjusted for the higher contrast needed to print in black and white, this may cause them to look grainy. 


\section{High-resolution holography - USAF resolution target}

The target was mounted on the translation stage, and tilted to minimize the shift of the image as much as possible when moving the target between the $\mathrm{S}$ and I planes. The portion of the target shown here includes Group 7, Element 6, a line from which is only $2.2 \mu \mathrm{m}$. The sharp edges of the lines implies that the lateral resolution of the system in this configuration is better than $1 \mu \mathrm{m}$.

Fig. 47, Direct Image $(88 \mu \mathrm{m}, 360$ pixels per side $)$ - USAF Resolution Target G7E4-6

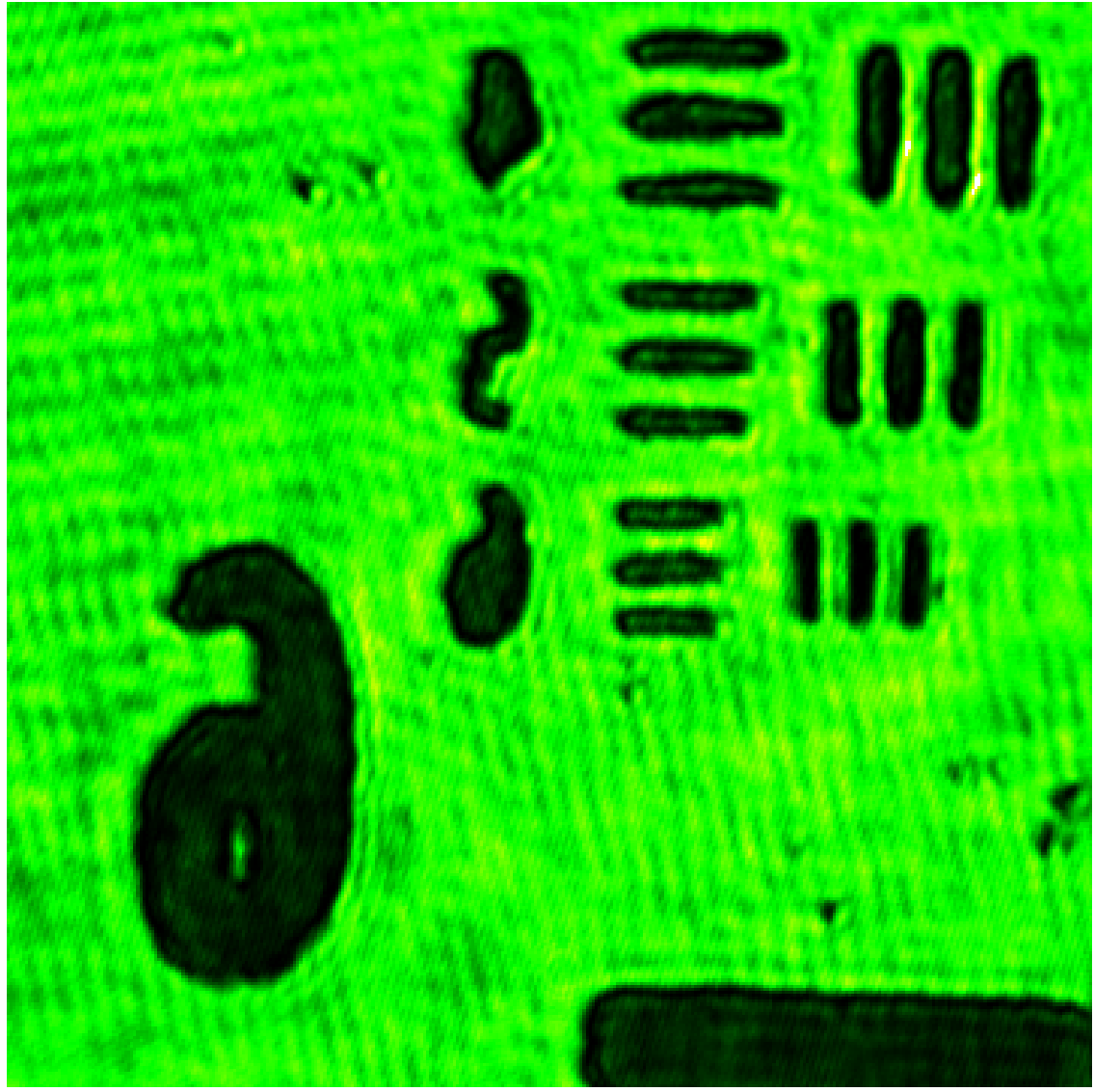


Fig. 48, Amplitude Image ( $88 \mu \mathrm{m}, 360$ pixels per side) - USAF Resolution Target G7E4-6

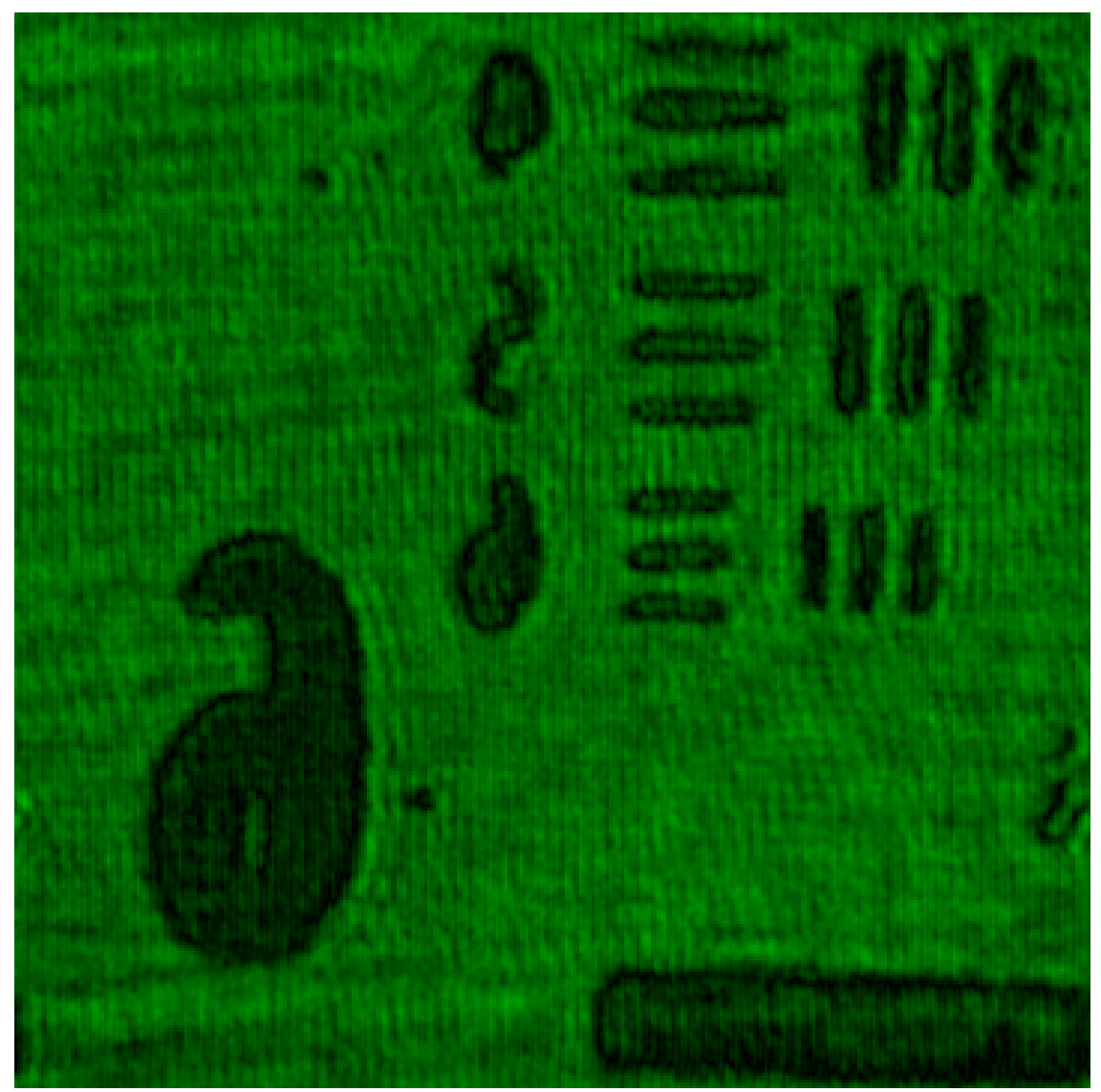

The amplitude reconstruction seen here is of fairly good quality, but the image is shifted slightly from the direct image. Specifically, the top line in G7E4 is not visible. This is most likely due to the astigmatism mentioned earlier. 
Fig. 49, Phase Image ( $88 \mu \mathrm{m}, 360$ pixels per side) - USAF Resolution Target G7E4-6

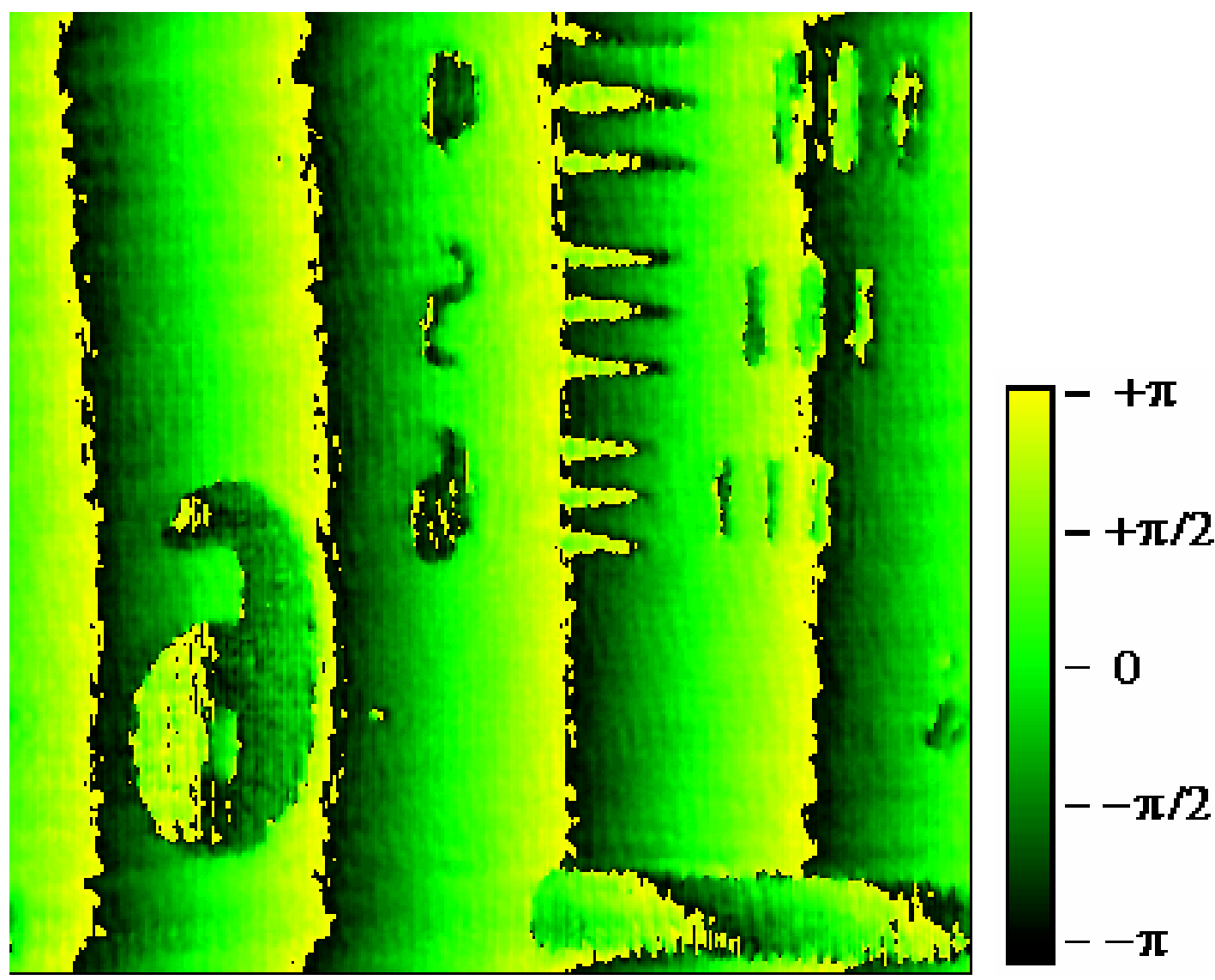

Measurements made of the depth of the metal film ranged from 120-160 nm.

Edmund Optics claims a depth of $100 \mathrm{~nm}$, "not tightly controlled" for the chromium film (Rosenfield [18]) ${ }^{15}$. Newport Optics (the particular target used was from Newport) claims a depth of $120-150 \mathrm{~nm}$ for the film [19]. 
Fig 50, 3D Rotated Image ( $88 \mu \mathrm{m}, 360$ pixels per side) with $2 \pi$ ambiguity - USAF Resolution Target G7E4-6

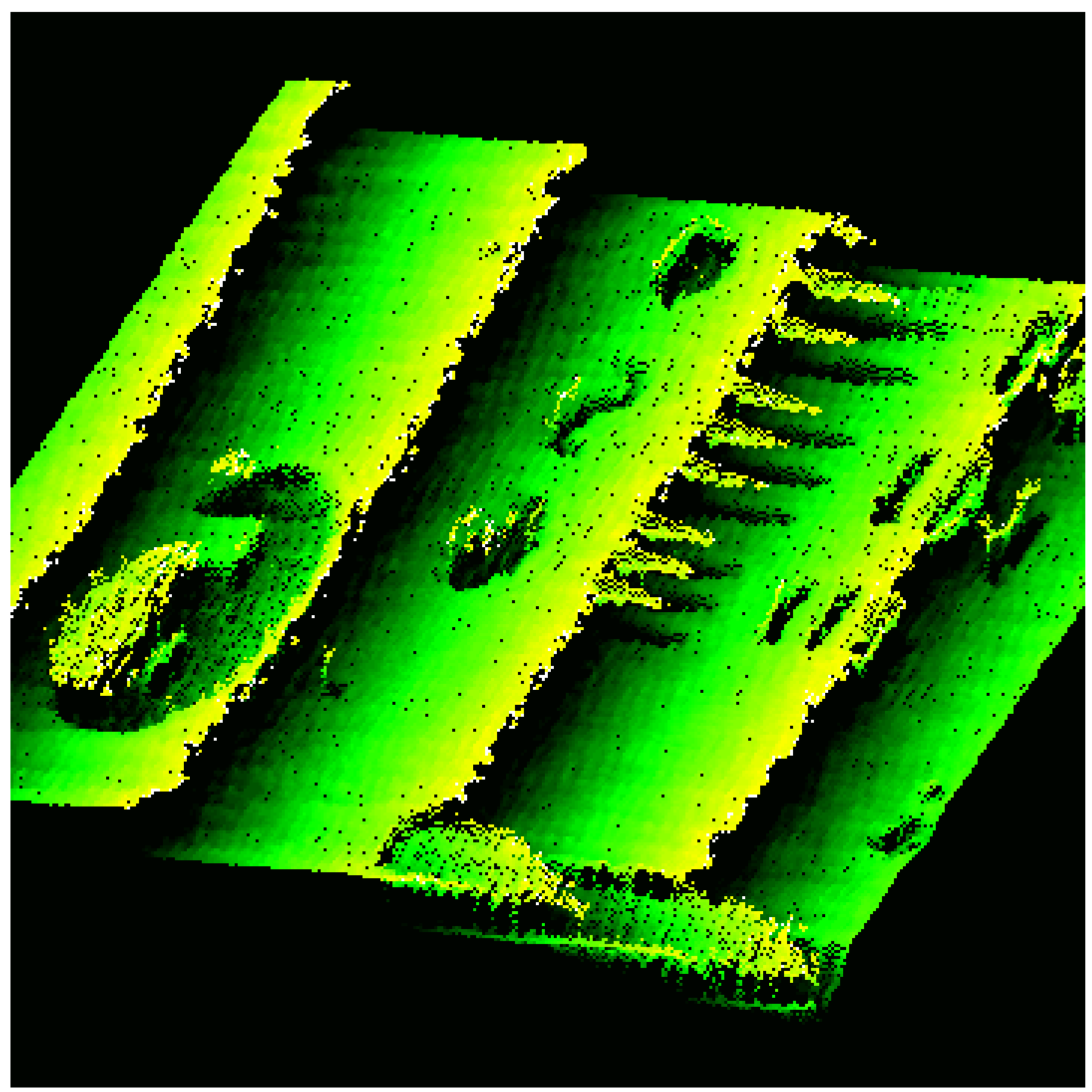

This is a rotated view of Fig. 49. Several of the lines appear to floating, in actuality, they are lower than the surrounding material, but this figure has not had the $2 \pi$ ambiguities removed. Since this figure only displays between $-\pi$ to $+\pi$, portions of the image slightly less than $-\pi$ are translated to just under $+\pi$. 


\section{High-resolution holography - onion cell 1 with nucleus}

This was the first high resolution image of a cell obtained in this project. The nucleus of the cell is clearly visible, as are the cell walls. The cell walls are noticeably thinner than in the lower resolution images; this is because, unbeknownst to the experimenter, the some of the cells used in the earlier experiments were already dead. Live cells can only be obtained from deeper inside the onion surface; they typically feature an intact nucleus and thin cells walls [14]. The nucleus is approximately $25 \mu \mathrm{m}$ across.

Fig. 51, Direct Image ( $88 \mu \mathrm{m}, 360$ pixels per side) - Onion cell

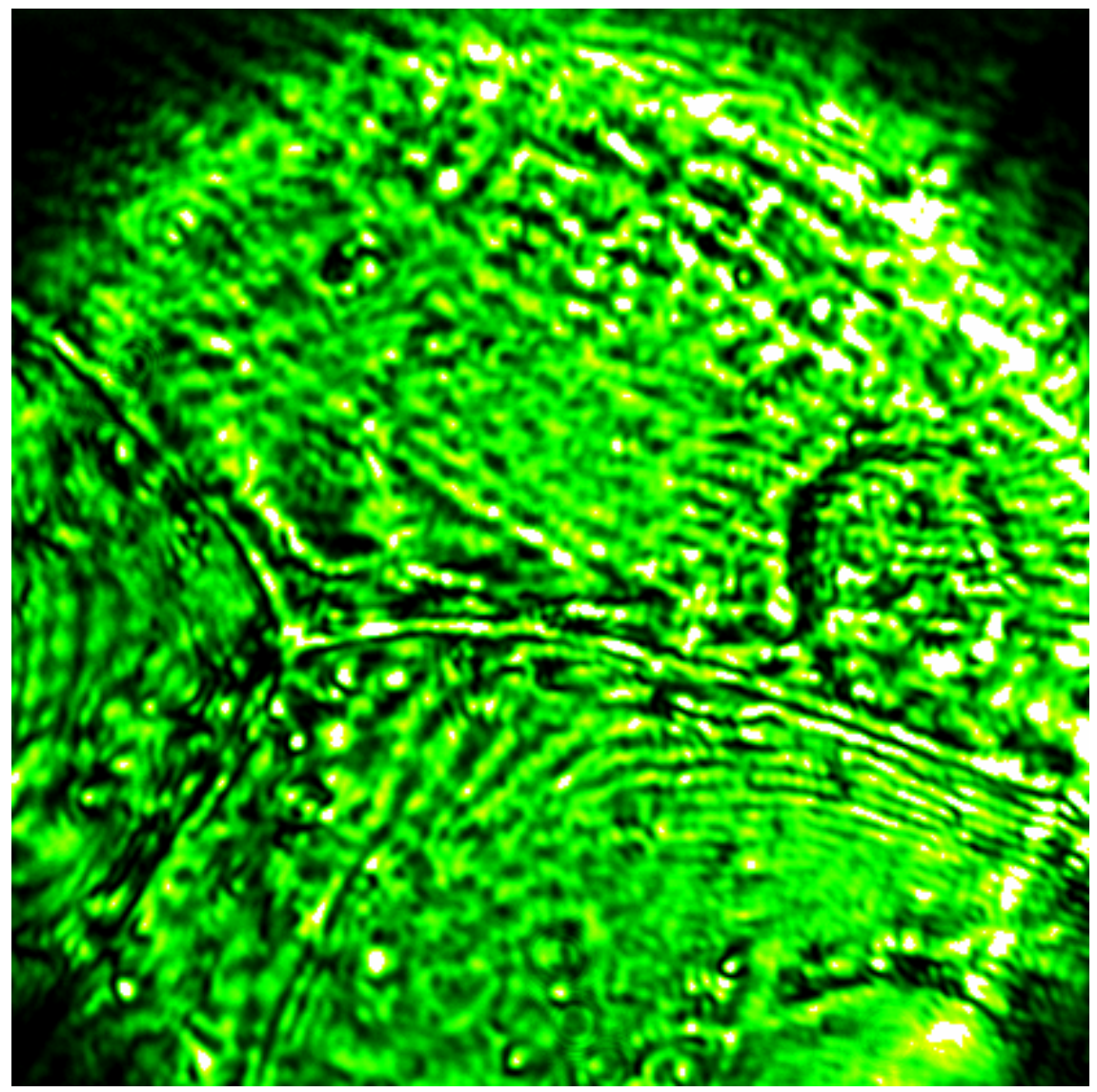


Fig. 52, Amplitude Image ( $88 \mu \mathrm{m}, 360$ pixels per side $)$ - Onion cell

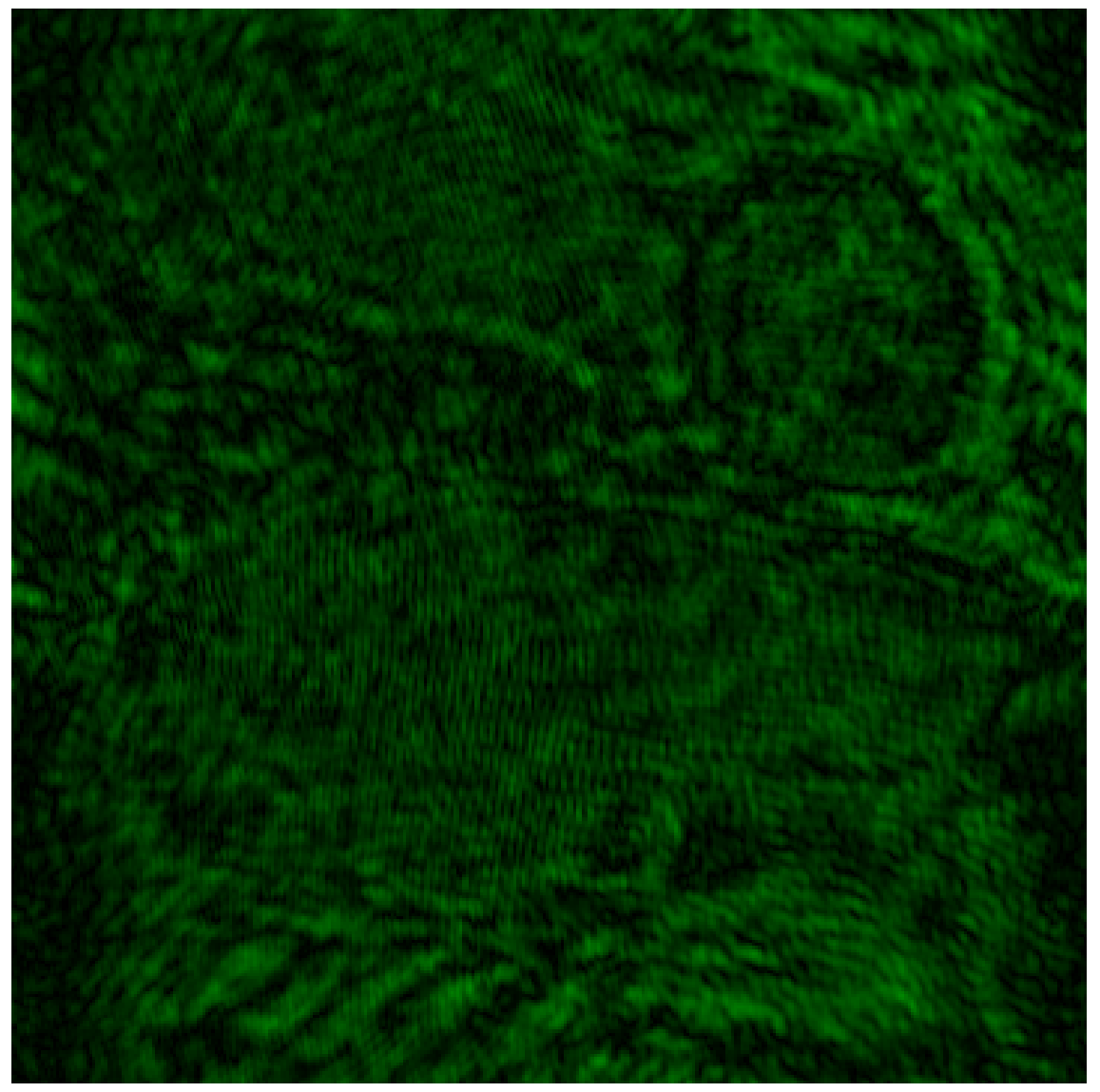

This amplitude reconstruction also displays the nucleus and cell walls. The image has been shifted due to the astigmatism. In addition, a slight magnification seems to have occurred, probably also due to the astigmatism. 
Fig. 53, Phase Image ( $88 \mu \mathrm{m}, 360$ pixels per side) - Onion cell

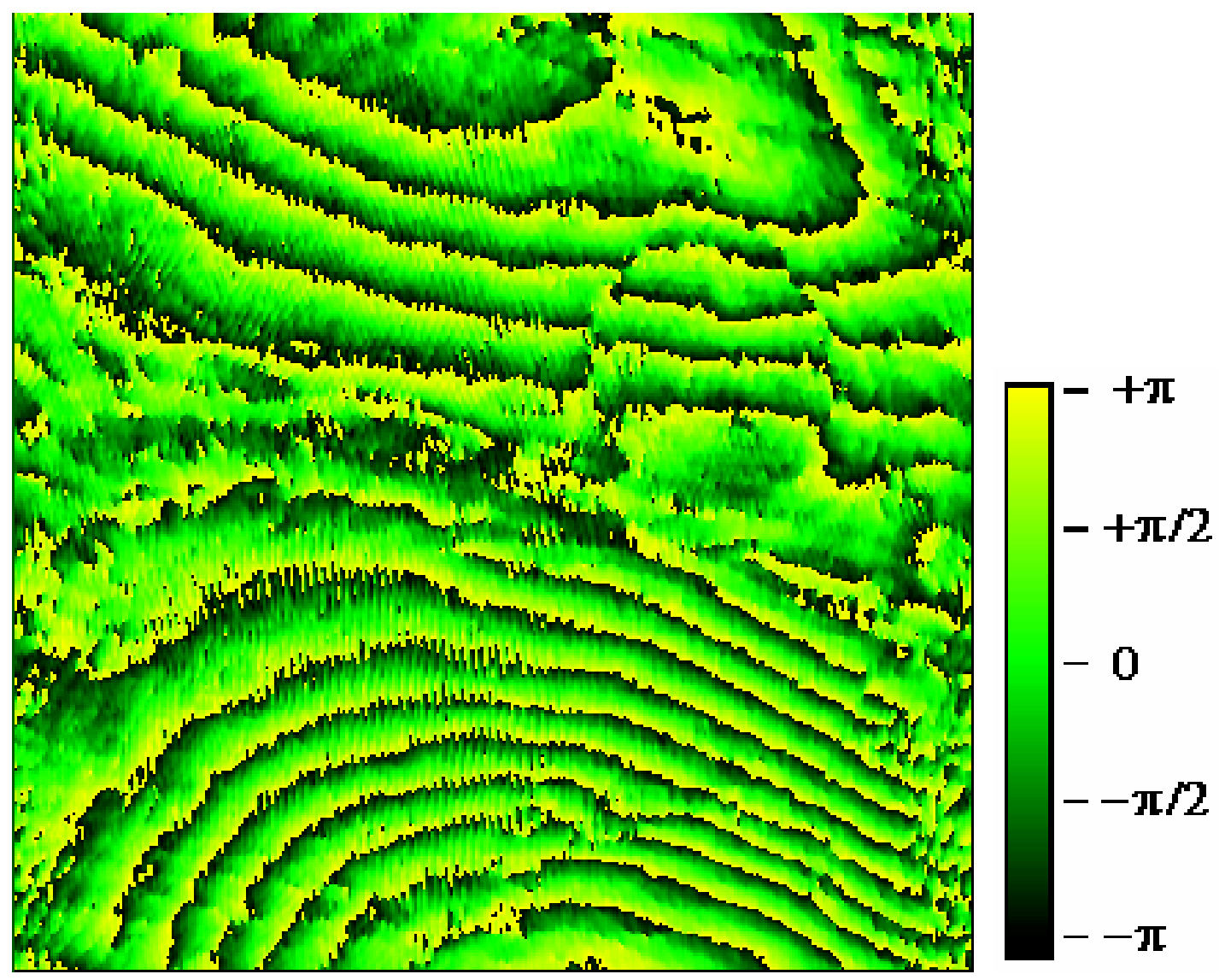

The phase image clearly shows the nucleus and cell walls, and the curved surface of the cells is easily discernable. The nucleus appears to have a different fringe spacing than the surrounding cell, possibly due to scattering off the surface of the nucleus, or more probably due to the increased refractive index within the nucleus. This will be established in future experiments. 


\section{High-resolution holography - onion cell 2 with nucleus}

This image was obtained without the use of an aperture. The cell wall and nucleus are clearly visible. Several other features are visible as well, it is possible these are cellular organelles. The nucleus is approximately $25 \mu \mathrm{m}$ across.

Fig. 54, Direct Image ( $88 \mu \mathrm{m}, 360$ pixels per side) - Onion cell with nucleus

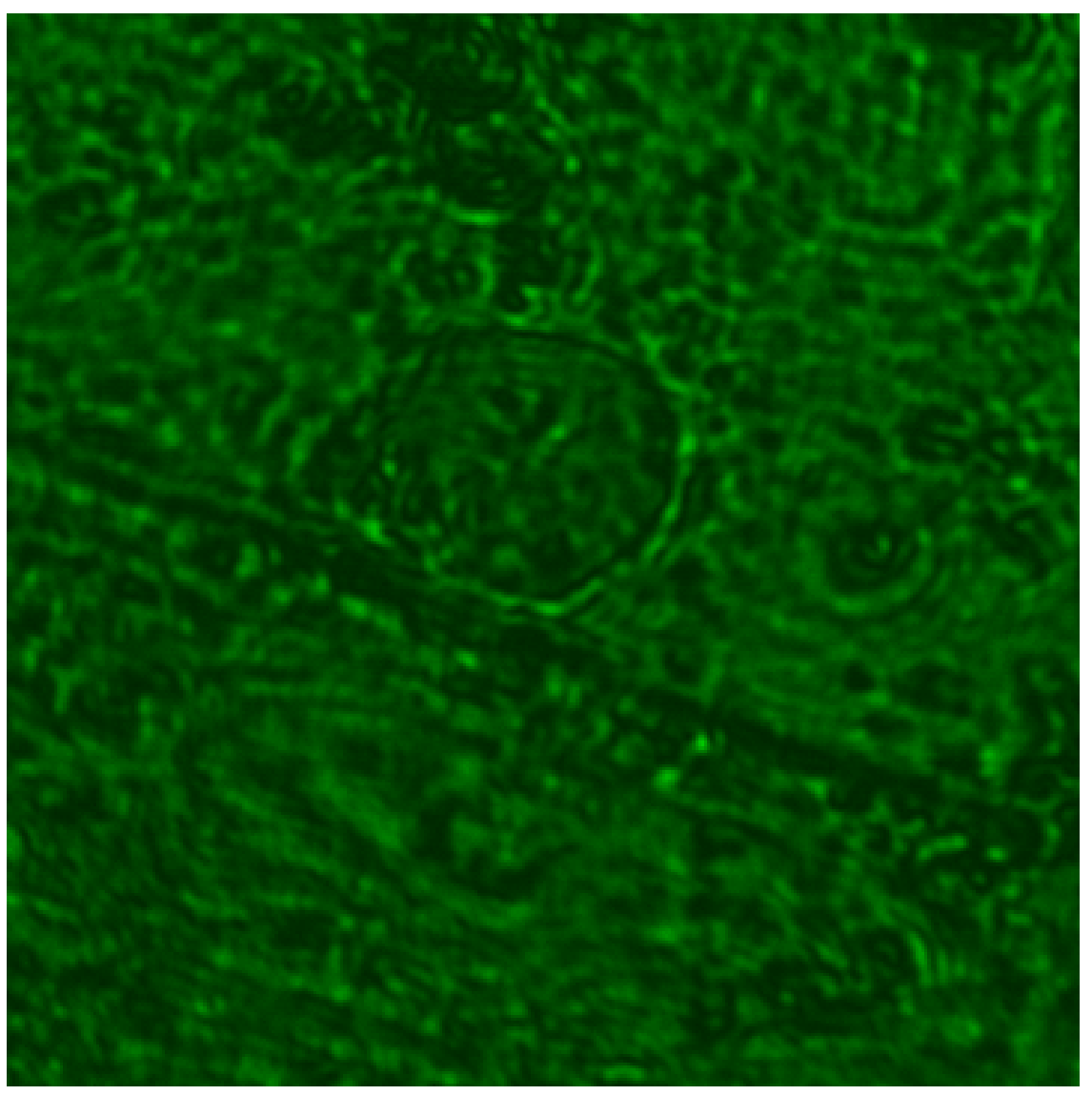


Fig. 55, Amplitude Image ( $88 \mu \mathrm{m}, 360$ pixels per side) - Onion cell with nucleus

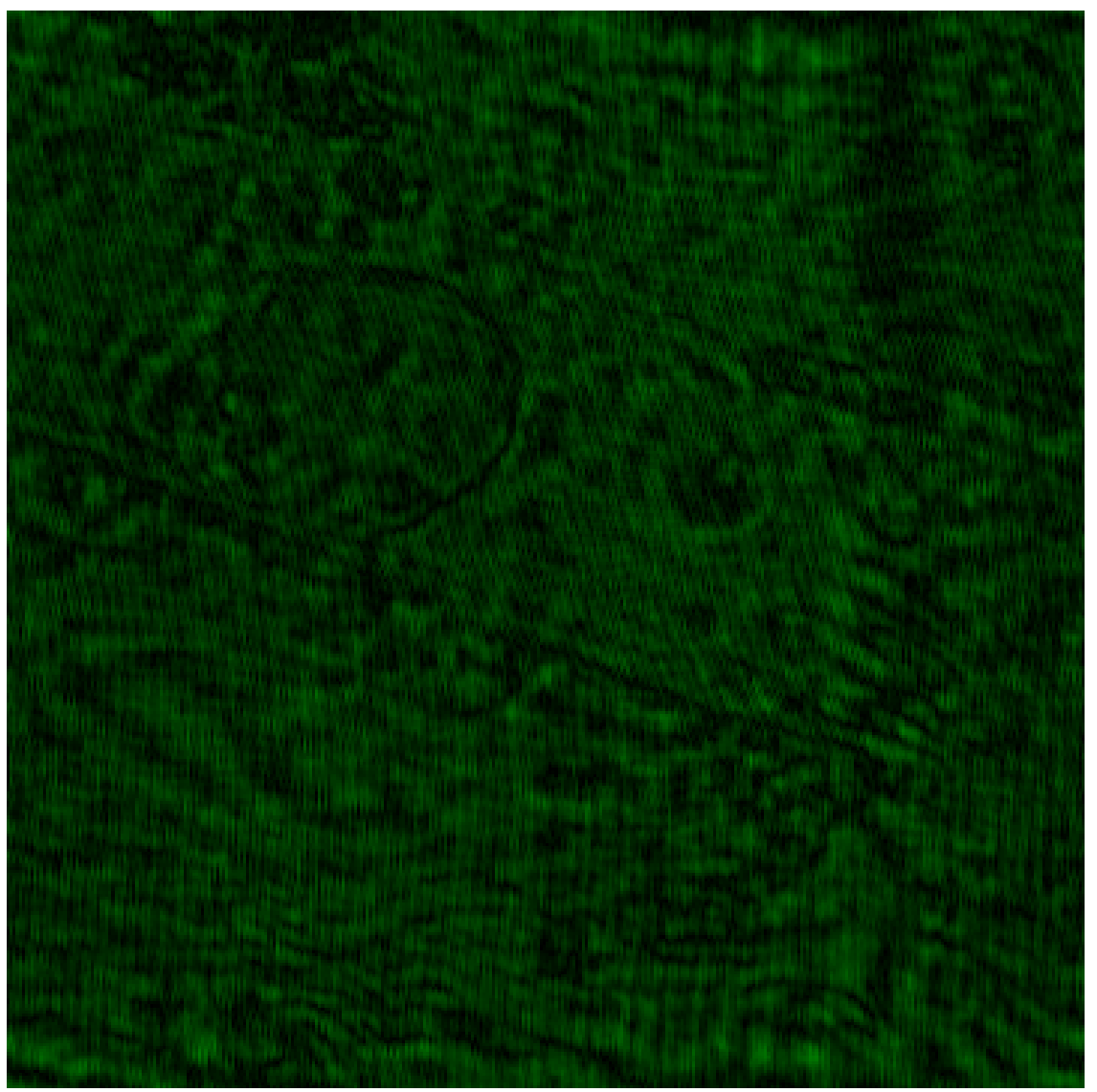

This image displays the wraparound effect discussed earlier. In spite of this, the image is fairly sharp and clear, the nucleus and cell wall are quite distinct. 
Fig. 56, Phase Image ( $88 \mu \mathrm{m}, 360$ pixels per side) - Onion cell with nucleus

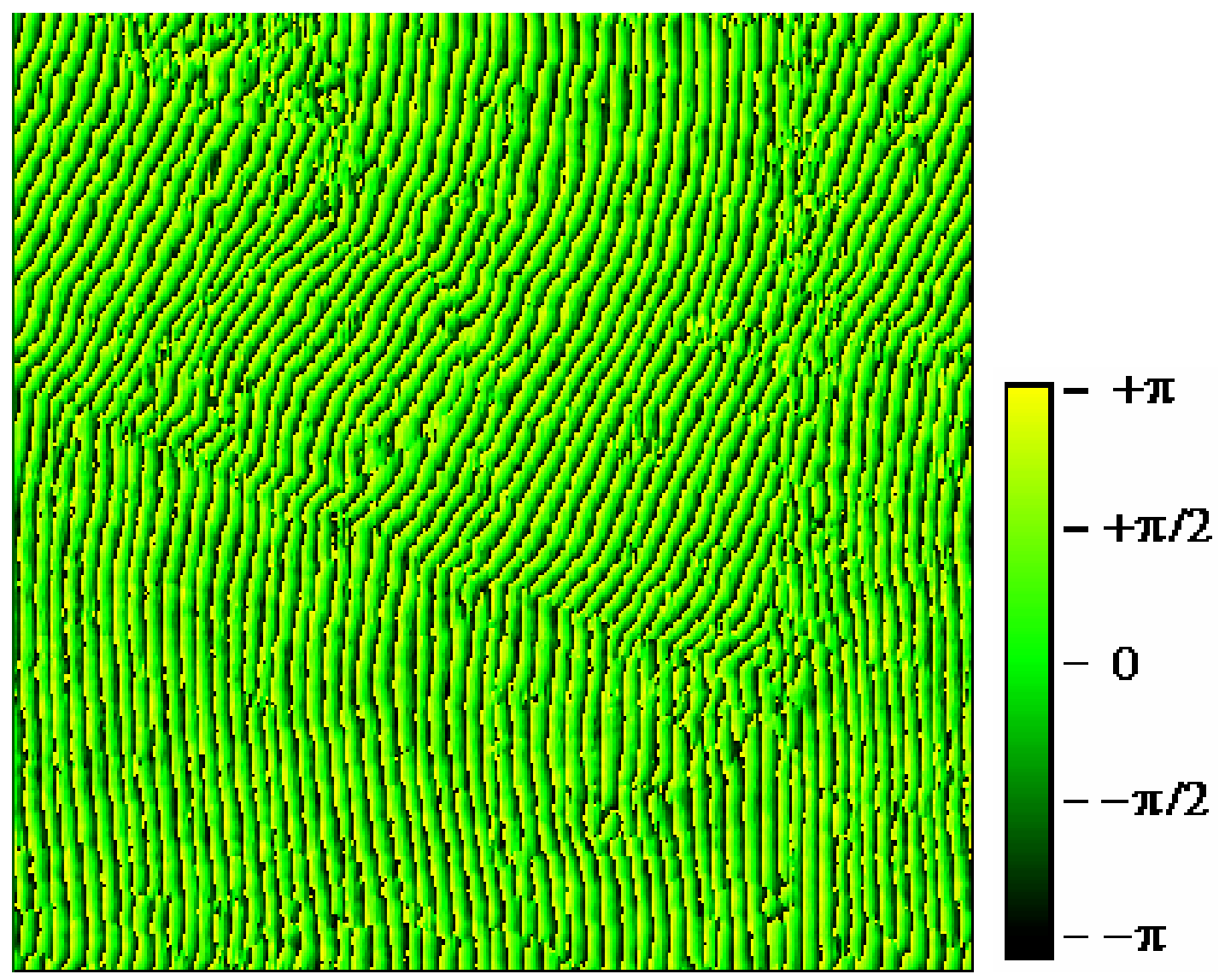

The phase image has high frequency fringes, these can be reduced by multiplying the phase of each pixel across the image by the factor $\mathrm{Cn \pi} / \mathrm{N}$, where $\mathrm{C}$ is the correction factor, $\mathrm{N}$ is the total number of pixels per side, and $\mathrm{n}$ is the column ${ }^{16}$. Nevertheless, the nucleus can still be seen. 


\section{High-resolution holography - Human blood cells 1}

These images of human blood cells were obtained by placing a drop of blood between a mirror and a cover slip ${ }^{17}$. The mirror was then mounted on the translation stage. The dark curvy line running through the center was a small air bubble. The presence of this bubble aided in bringing the image into focus. This image also displays the "wraparound" effect. The average diameter of a human blood cell is $7 \mu \mathrm{m}$.

Fig. 57, Direct Image ( $88 \mu \mathrm{m}, 360$ pixels per side) - Human blood cells

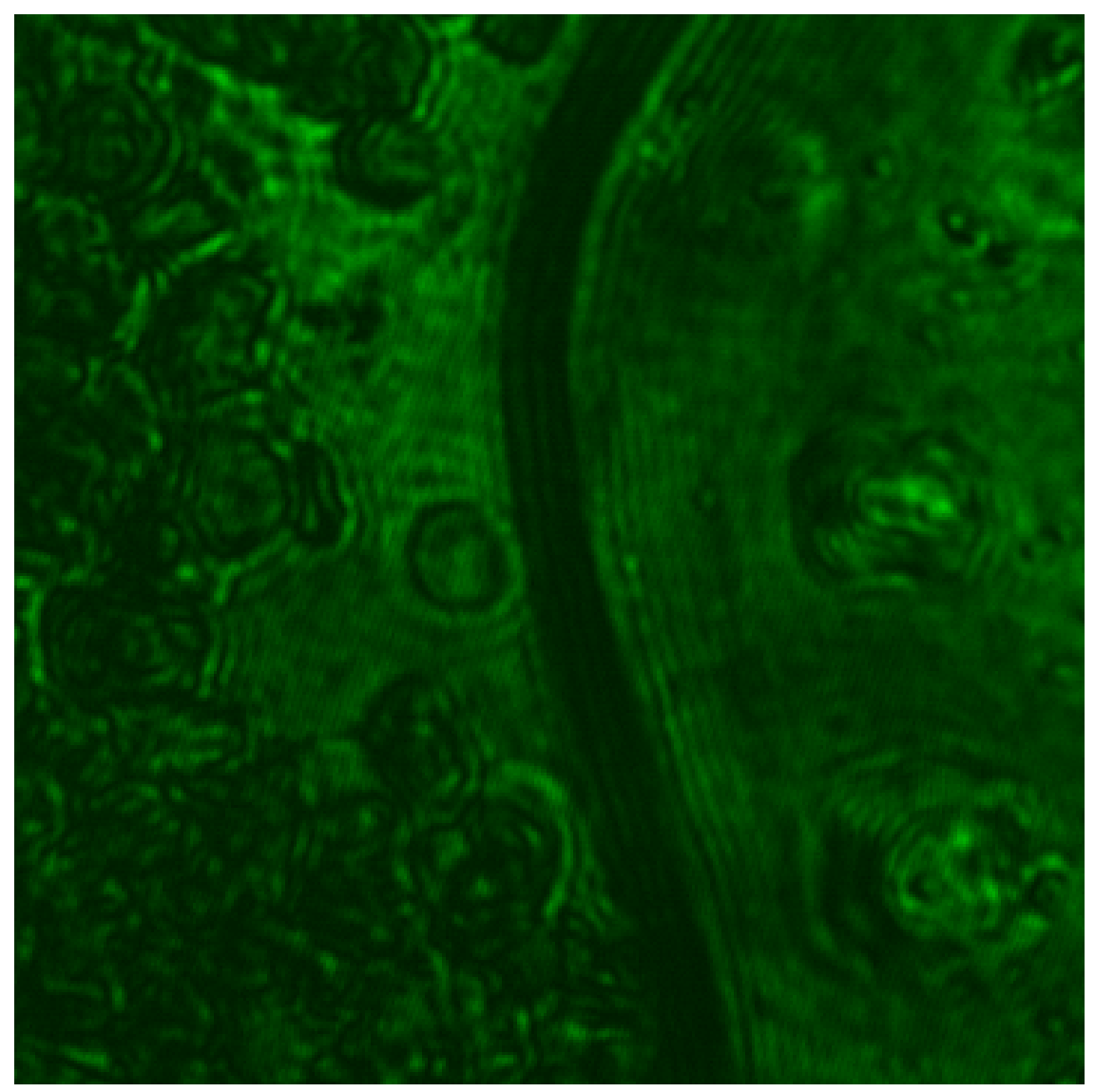


Fig 58, Amplitude Image ( $88 \mu \mathrm{m}, 360$ pixels per side $)$ - Human blood cells

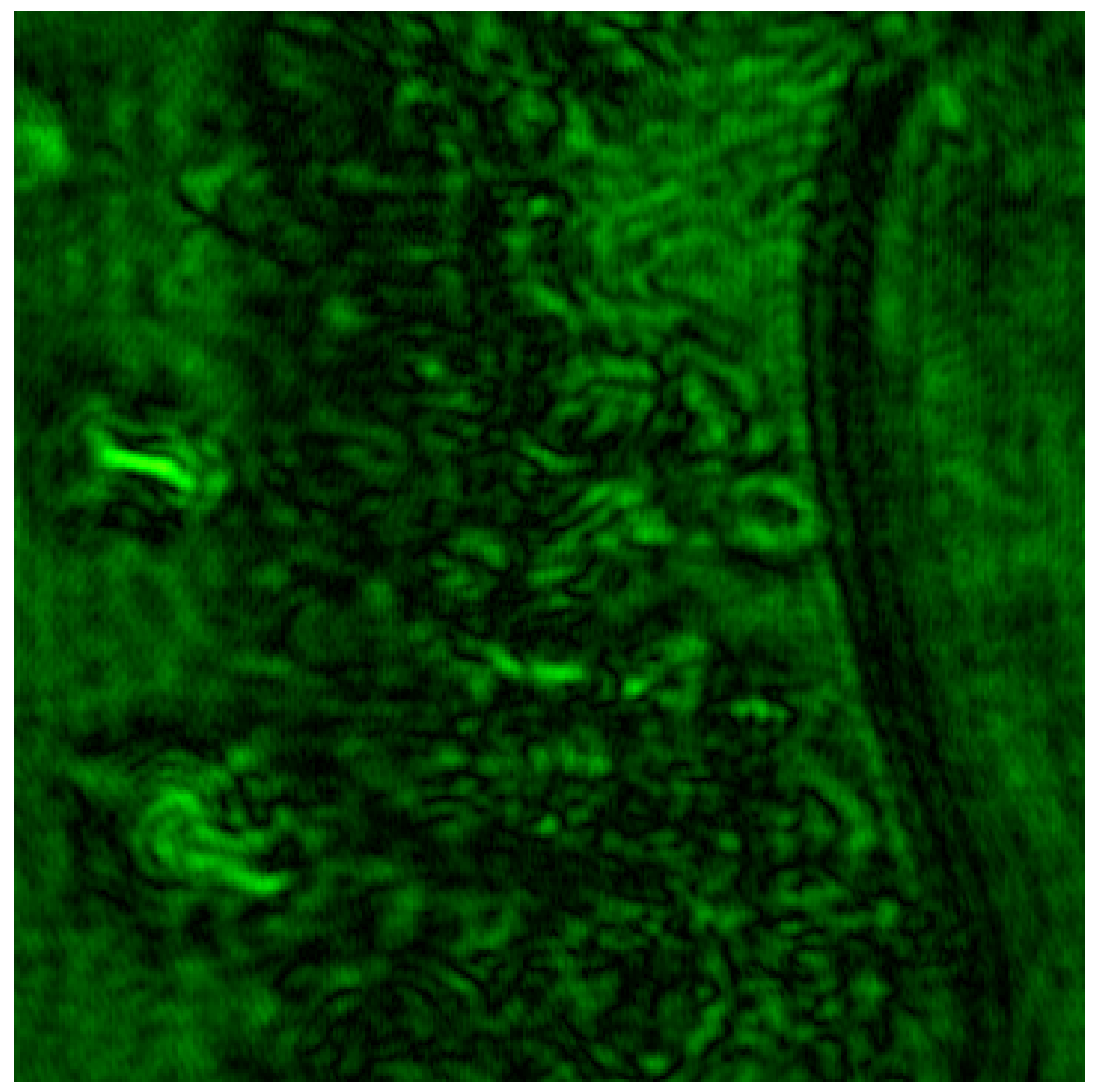

These images are fairly noisy, because the blood cells moved as the area under illumination heated up due to the incident laser light. The cell in the center of the image was relatively still, and so it was selected to be the focus of this image.

Like many of the other images acquired using the microscope objective, this one suffers from "wraparound". 
Fig. 59, Phase Image ( $88 \mu \mathrm{m}, 360$ pixels per side $)$ - Human blood cells

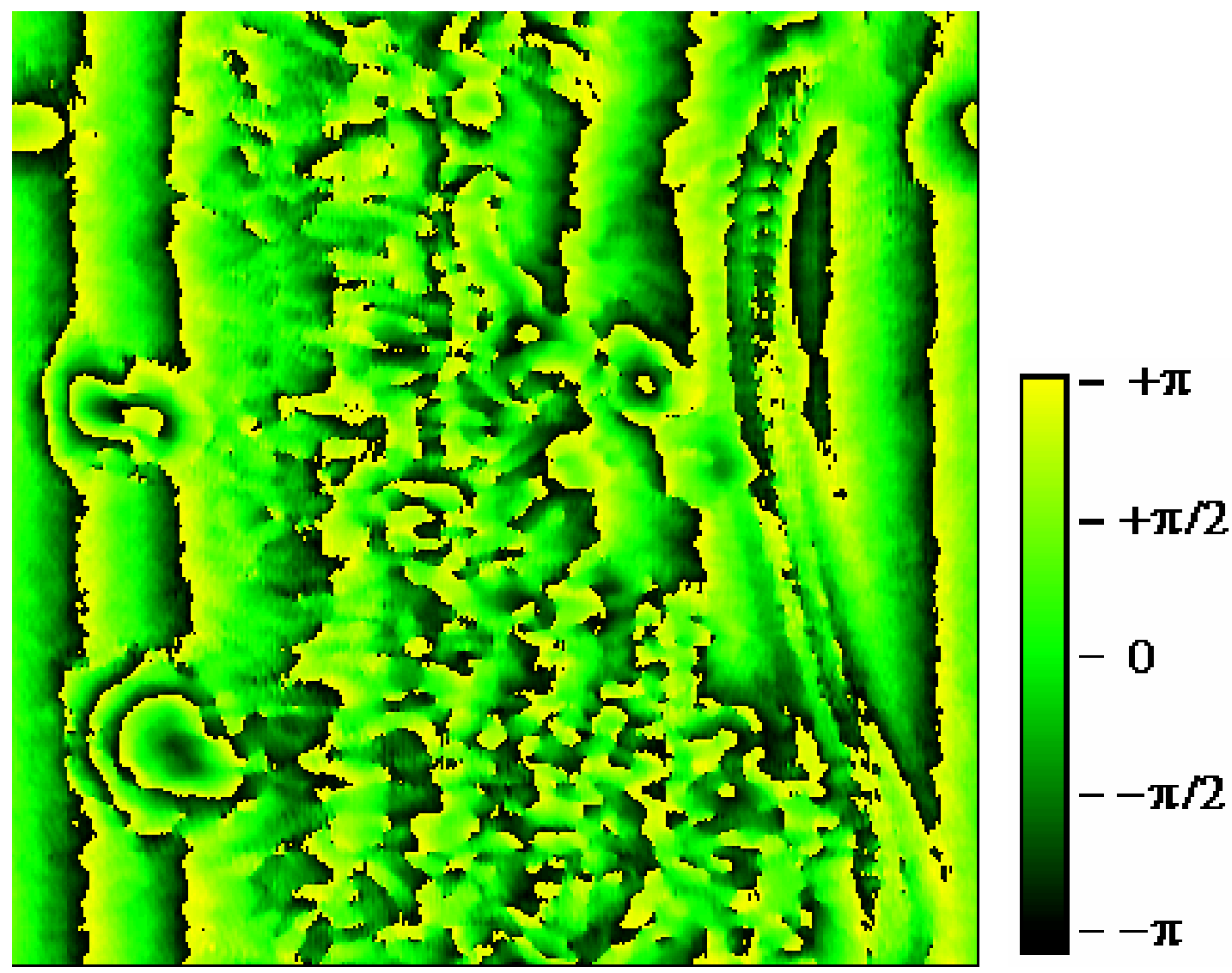

The central blood cell is quite visible, and the divot in the center of the cell happens to occur right around the $2 \pi$ ambiguity, which makes it particularly obvious. Since the system can resolve features as small as $2 \mu \mathrm{m}$, it is quite reasonable to think that the divot should be distinguishable. 


\section{High-resolution holography - Human blood cells}

This image was from a different portion of the same mounted sample as the previous images. The air bubble is now on the left side of the image, and a blood cell in the center clearly displays the classical blood cell structure seen in Appendix A, Fig. A5. The long structures are blood cells, on their sides, that have stacked like a pile of coins.

Fig. 60, Direct Image $(88 \mu \mathrm{m}, 360$ pixels per side $)$ - Human blood cells

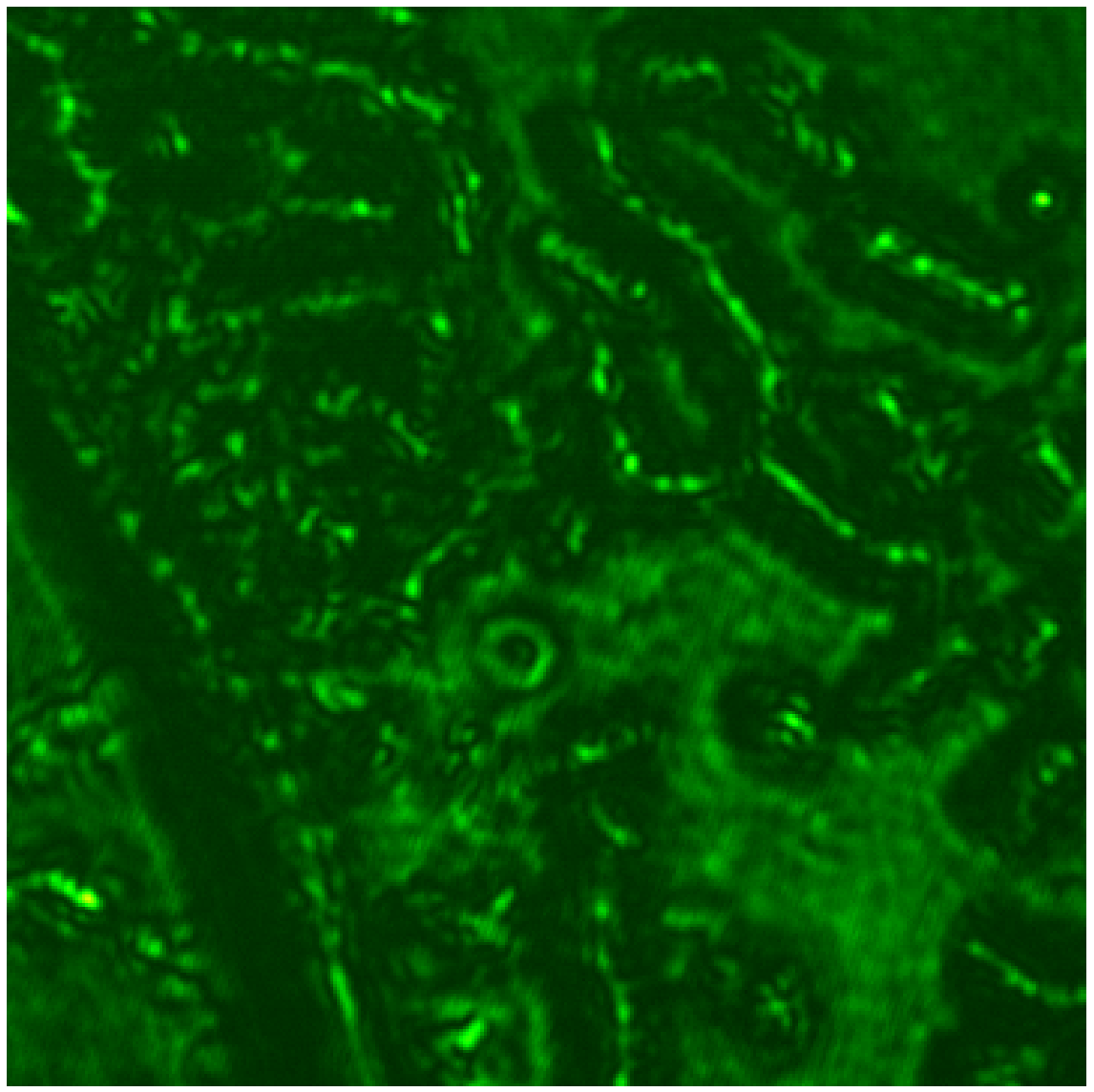


Fig. 61, Amplitude Image ( $88 \mu \mathrm{m}, 360$ pixels per side) - Human blood cells

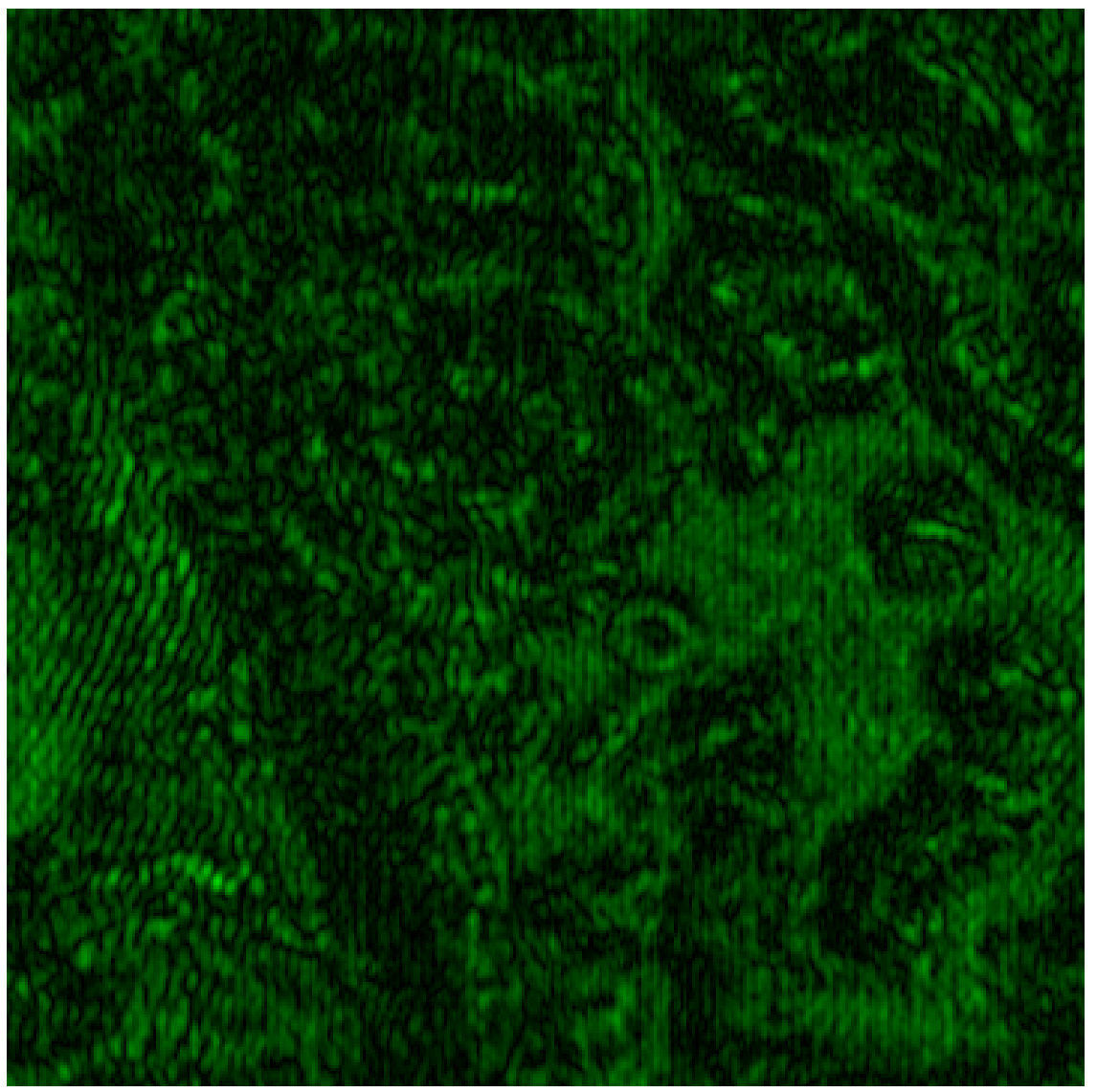

The blood cell in the center is clearly visible, and some of the stacked blood cells can be made out as well. This is one of the few images that did not display the wraparound effect. Most of the motion from the blood cells was on the edge closest to the bubble, where the image is blurriest. 
Fig. 62, Phase Image ( $88 \mu \mathrm{m}, 360$ pixels per side $)$ - Human blood cells

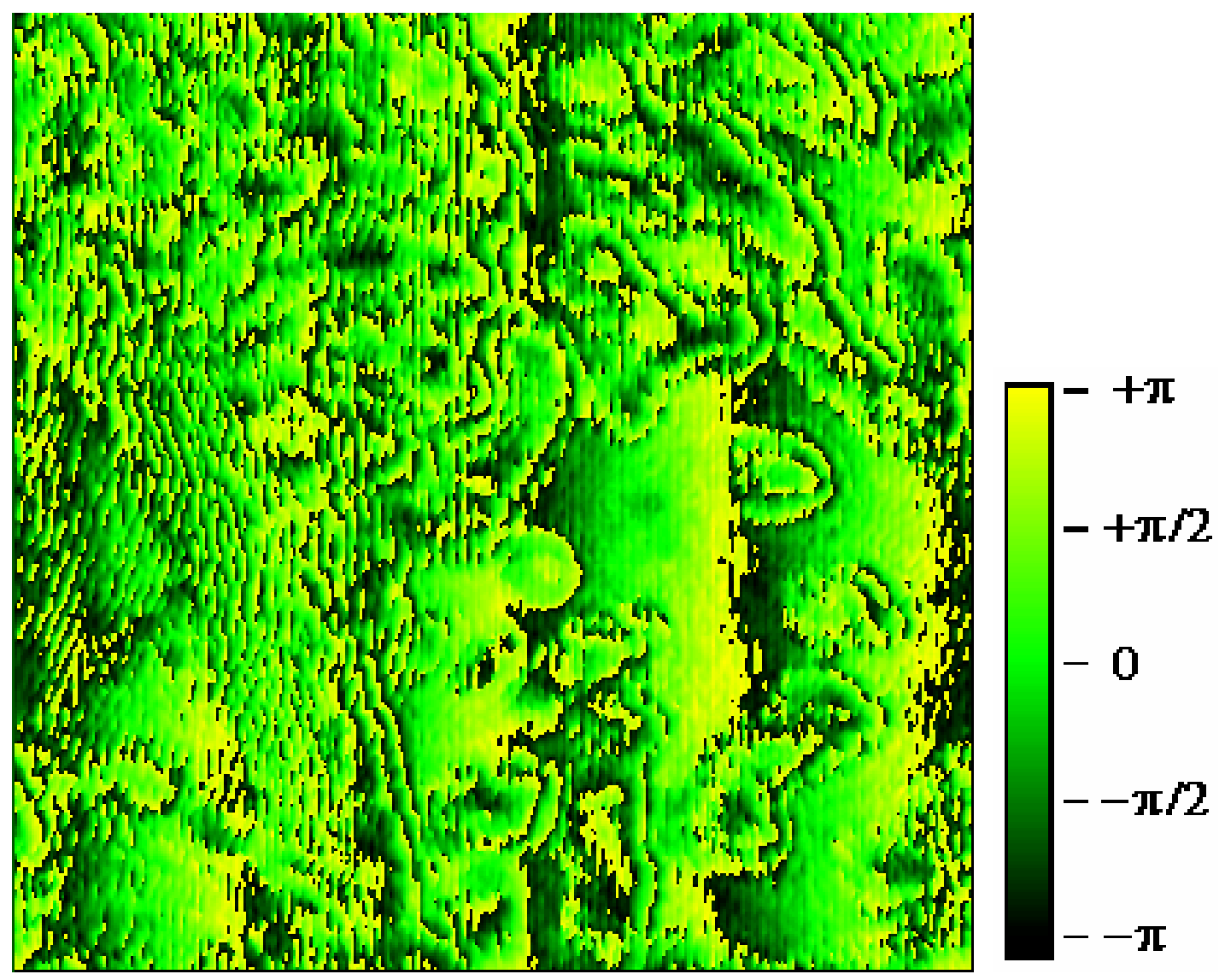

The phase image clearly shows the central blood cell and the indentation in the center. Some of the stacked blood cells can be seen, but not clearly. The region on the left corresponds to the region that had blood cells moving the most while this image was being acquired. 


\section{CONCLUSIONS}

The application of phase imaging holography to biological samples has been demonstrated. The multiple wavelength technique for the elimination of $2 \pi$ ambiguities has also been shown. In the final setup, a lateral resolution of better than $2 \mu \mathrm{m}$ and an axial resolution of approximately $10 \mathrm{~nm}$ has been achieved. This work may allow future researchers to accurately and easily image subcellular features. The technique works across a broad range of input parameters, and may be readily adaptable to real world conditions.

There are several directions for future work in this area. First is the addition of higher quality optical components to remove the astigmatism caused by the tilting of the microscope objective lens. Obtaining holograms of a sample mounted on a non-reflective surface is another. Finally, preliminary work suggests that it may be possible to use a series of holograms from a sample mounted on reflective and non-reflective surfaces to obtain a complete 3 dimensional mapping of the cell, including index variations throughout the cellular material. Such a technique might be of enormous benefit to researchers in the biophysics field, as currently the only method to see how cells attach to a substrate involves the removal of those very cells (Giaever [20]). 


\section{REFERENCES}

[1] D Gabor, "A new microscope principle", Nature 161, 777-8 (1948)

[2] E. Leith \& J. Upatnieks, "Reconstructed wavefronts and communication theory”, J. Opt. Soc. Am. 52, 1123 (1962)

[3] R. Powell \& K. Stetson, "Interferometric vibration analysis by wavefront reconstruction”, J. Opt. Soc. Am. 55, 1593 (1965)

[4] A. Lohmann, D. Paris, "Binary Fraunhofer holograms generated by computer", Appl. Opt. 6, 1739 (1967)

[5] U. Schnars, M. Thomas, and W. Jueptner, "Digital recording and numerical reconstruction of holograms: reduction of the spatial frequency spectrum", Opt. Eng. 35, 977 (1996)

[6] U. Schnars and W. Jueptner, "Digital recording and numerical reconstruction of holograms", Measurement Sci. Technol. 13, R85 (2002).

[7] M. K. Kim, "Tomographic three-dimensional imaging of a biological specimen using wavelength-scannin digital interference holography", Opt. Expess 7, 305 (2000), http://www.opticsexpress.org

[8] W. Press, B. Flannerly, S. Teukolsky, W. Vetterling, Numerical Recipes (Cambridge University Press, Cambridge, 1986) p. 392, 387

[9] M. Servin, L. Marroquin, D. Malacar, F. Cuevas, "Phase unwrapping with a regularized phase-tracking system", Appl. Opt. 37, 1917 (1998)

[10] J. Gass, A. Dakoff, and M. K. Kim, "Phase imaging without $2 \pi$ ambiguity by multiwavelength digital holography", Opt. Lett. 28, 1141 (2003)

[11] E, Cuche, F. Bevilacqua, and C. Depeursinge, "Digital holography for quantitative phase-contrast imaging", Opt. Lett. 24, 291 (1999)

[12] H. Gausman, W. Allen, and D. Escobar, "Refractive index of plant cell walls", Appl. Opt. 13, 109 (1974)

[13] F. Lanni, A. Waggoner, and D. Taylor, "Structural organization of interphase 3T3 fibroblasts studied by total internal reflection fluorescence microscopy", Journal of Cell Biology 100, 1091 (1985)

[14] E. Matthews, University of South Florida, Department of Biology; February 2004 (private communication) 
[15] D. Parshall and M. K. Kim, "Phase imaging digital holography for biological microscopy", OISE (Orlando, 2003)

[16] J. Goodman, Introduction to Fourier Optics (McGraw-Hill, San Francisco, 1968)

[17] M.K. Kim and D. Parshall, "Phase imaging digital holography for biological microscopy", SPIE Photonics West, BiOS 5324 (San Jose, 2004)

[18] R. Rosenfield, Edmund Optics, Technical Support; March 2004 (private communication)

[19] Heather Cmiel, Newport Optics, Technical Support; March 2004 (private communication)

[20] I. Giaever, President, Applied Biophysics, Inc.; October 2003 (private communication)

[21] J. Gass, University of South Florida, Department of Physics; March 2004 (private communication) 
APPENDICES 


\section{Appendix A (Comparison images)}

The following images of various cells were all taken with a National DC-2 Digital Microscope. Motic Images 2000 was used to acquire the data to computer.

These images should provide a comparison for the results presented elsewhere in the thesis.

Fig. A1, Onion cell with nucleus (1000X)

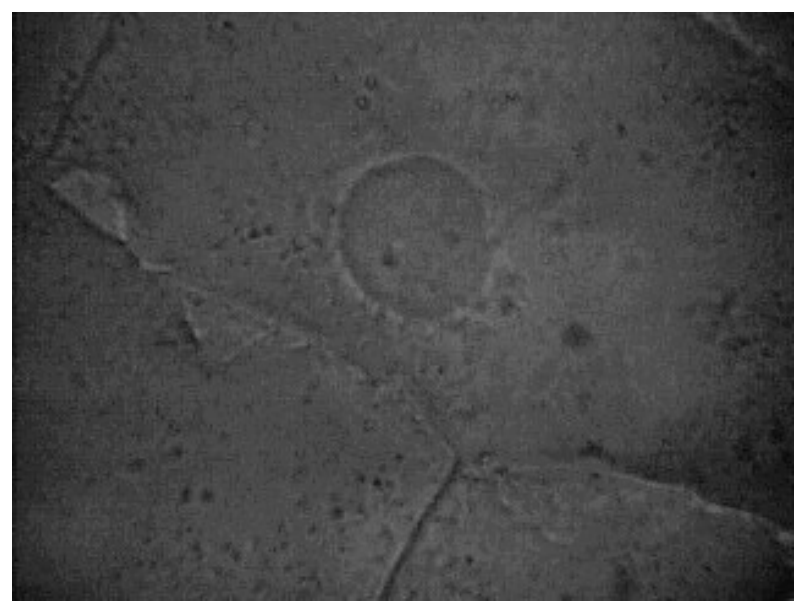

Fig. A2, Onion cell, lysing nucleus (1000X)

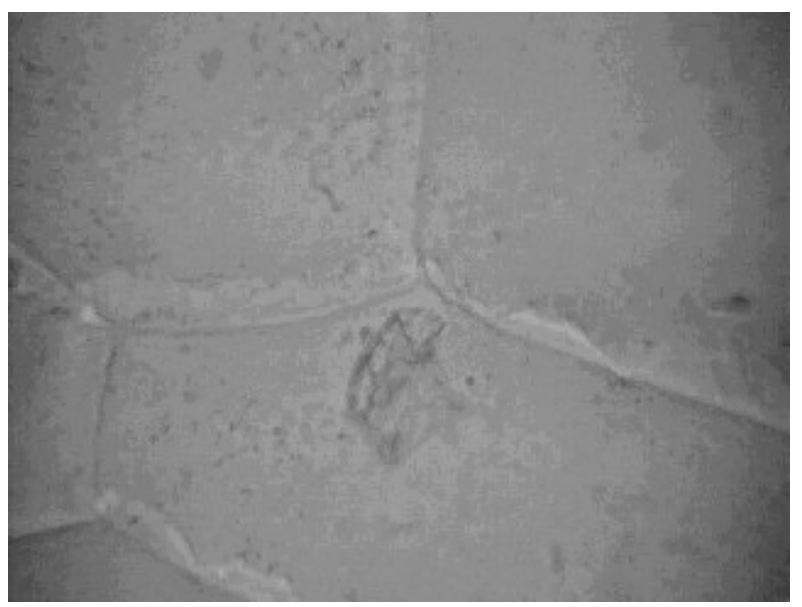


Appendix A (Continued)

Fig. A3, Onion cells, slightly decomposed (1000X)

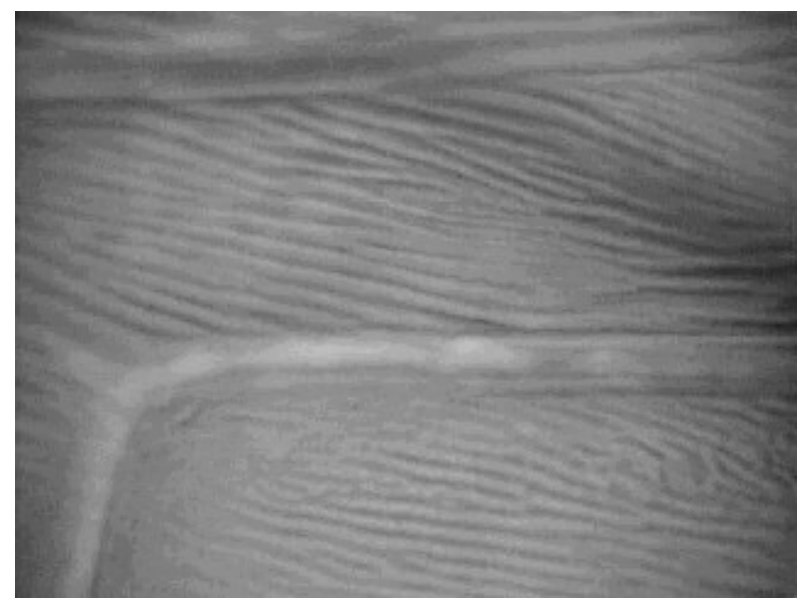

Fig. A4, Onion cells, slightly decomposed (400X)

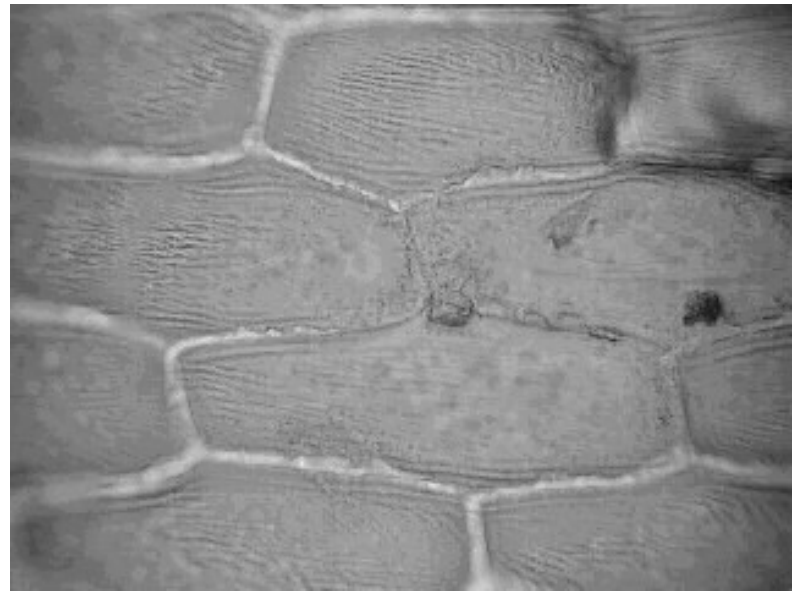

Fig. A5, Human blood cells (1000X)

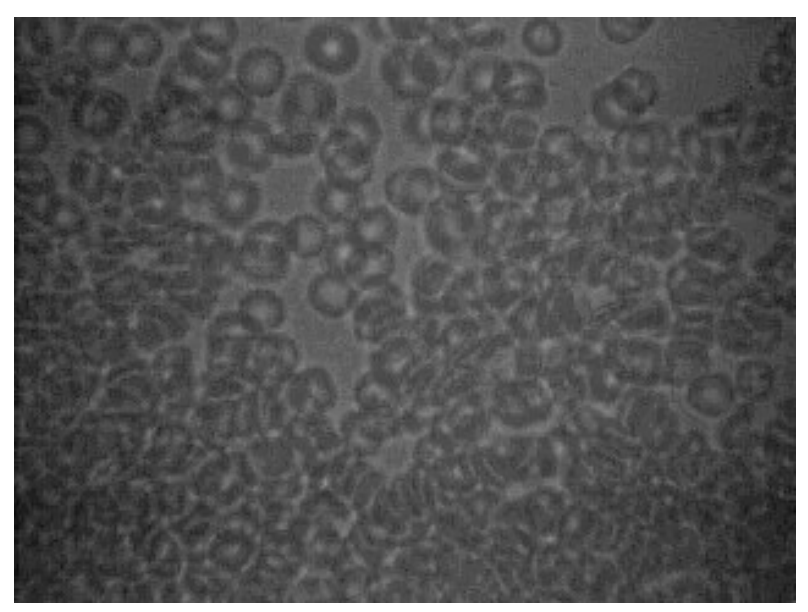


Appendix B (Potential application)

A transmission hologram, as described in this thesis, provides a great deal of information about the size and shape of a cell; included in that information is a description of the back surface of the cell. However, this information is mixed in with information about the front surface and body of the cell. If it were possible to separate these three pieces of information, a full three dimensional map of the cell could be created.

To obtain information about the front of the cell, it is necessary to take a reflection hologram, as opposed to a transmission hologram. In a reflection hologram, the incident light returns from the interface between the air and front surface of the cell, and possibly even the interface between the body and back surface of the cell. Such a scenario allows us to isolate the three pieces of information given in a transmission hologram and determine each of the independently.

If the target is mounted as pictured below, then there are three regions that are of interest. In this case, $a(x, y)$ is the distance between the front surface of the cell and an arbitrary plane in space; the factor $n(x, y) b(x, y)$ is the width of the cell, $b(x, y)$, times the index of the cell at any given point, $n(x, y)$. Finally, $c(x, y)$ is the distance between the back surface of the cell and the substrate. The substrate will be considered flat over the very small range we are examining. 


\section{Appendix B (Continued)}

After processing the diffraction of the hologram, the result is a $2 \mathrm{D}$ array of

numbers of the form $H(x, y)=r(x, y) e^{i \theta(x, y)}$, where $H(x, y)$ is the holographic reconstruction, and $r(x, y)$ and $\theta(x, y)$ are the values of the amplitude and phase calculated at each pixel. This value $H(x, y)$ is the sum of each of the waves being reflected off the sample. In the case of the transmission hologram, only one wave is recorded; the wave takes the form

$$
H_{T}(x, y)=r(x, y) e^{i k(a(x, y)+n(x, y) b(x, y)+c(x, y))}
$$

Here the subscript $\mathrm{T}$ denotes a transmission hologram, and $\mathrm{k}$ is the wave vector. The phase of this hologram is

$$
H_{T \theta}(x, y)=k(a(x, y)+n(x, y) b(x, y)+c(x, y))
$$

By comparing this result to a reflection hologram such as the one seen in Fig. B1 below, as well as a hologram taken with no sample mounted, we can isolate the different terms.

Fig. B1, Reflection holography

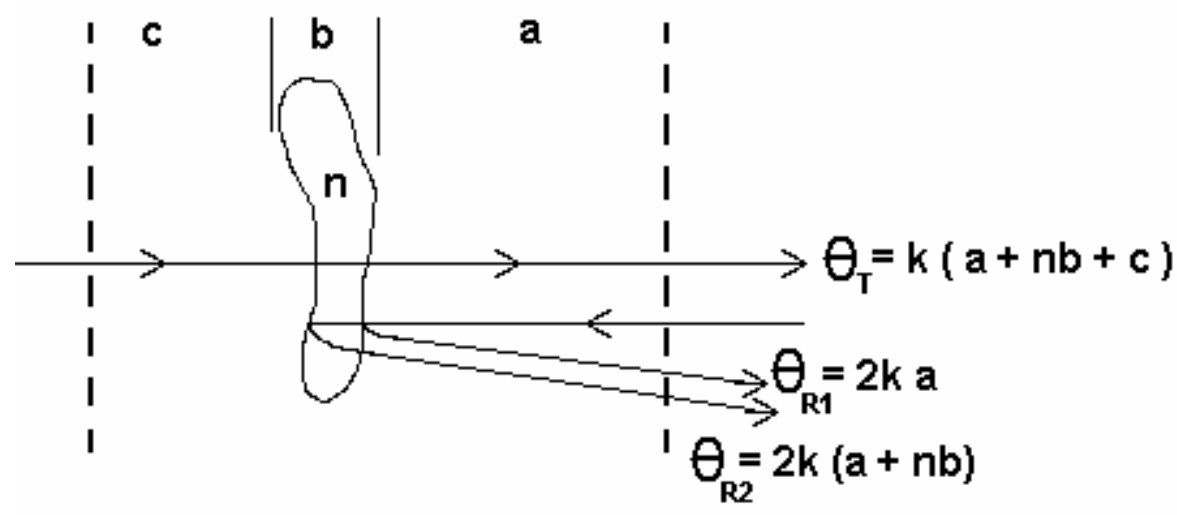




\section{Appendix B (Continued)}

As can be seen in the image, a reflection hologram should consist of two terms, one corresponding to reflection from the front surface of the sample, the other corresponding to reflection from the back surface. The ratio of reflection to incident light is given by

$$
R=\left|\frac{n_{1}-n_{2}}{n_{1}+n_{2}}\right|^{2}
$$

Taking $n_{1}=1.0$ for air, and $n_{2}=1.4$ for a plant cell wall [12], the reflection is somewhat less than $3 \%$. While small, this is more than enough to use the holography system described in this thesis without modification, and furthermore, small enough to make the assumption that the amplitude of the light reflected from the front surface is equal to the light reflected from the back surface. Thus, the total reflected light can be written as

$$
H_{R}(x, y)=A(x, y)\left(e^{i 2 k a(x, y)}+e^{i 2 k(a(x, y)+n(x, y) b(x, y))}\right)
$$

or

$$
H_{R}(x, y)=A(x, y) e^{i 2 k a(x, y)}\left(1+e^{i 2 k(2(x, y) b(x, y))}\right)
$$

if we multiply by the complex conjugate, the result is

$$
H_{R}^{*}(x, y) H_{R}(x, y)=A^{2}(x, y)(2+2 \cos (n(x, y) b(x, y))
$$

thus eliminating the $a(x, y)$ term. Therefore, the value of the path length through the sample can be found from

$$
n(x, y) b(x, y)=1 / 2\left(\cos ^{-1}\left(\frac{H_{R}^{*}(x, y) H_{R}(x, y)}{r^{2}(x, y)}-2\right)\right)
$$




\section{Appendix B (Continued)}

where the $r^{2}(x, y)$ term from equation $\mathrm{B} 1.1$ is substituted for the $A^{2}(x, y)$ term that would otherwise be expected. This is to ensure that any opaqueness in the sample is accounted for. The result of equation B1.4 for a sample of onion cells mounted without a backing is displayed below.

Fig. B2, Optical path length through cells

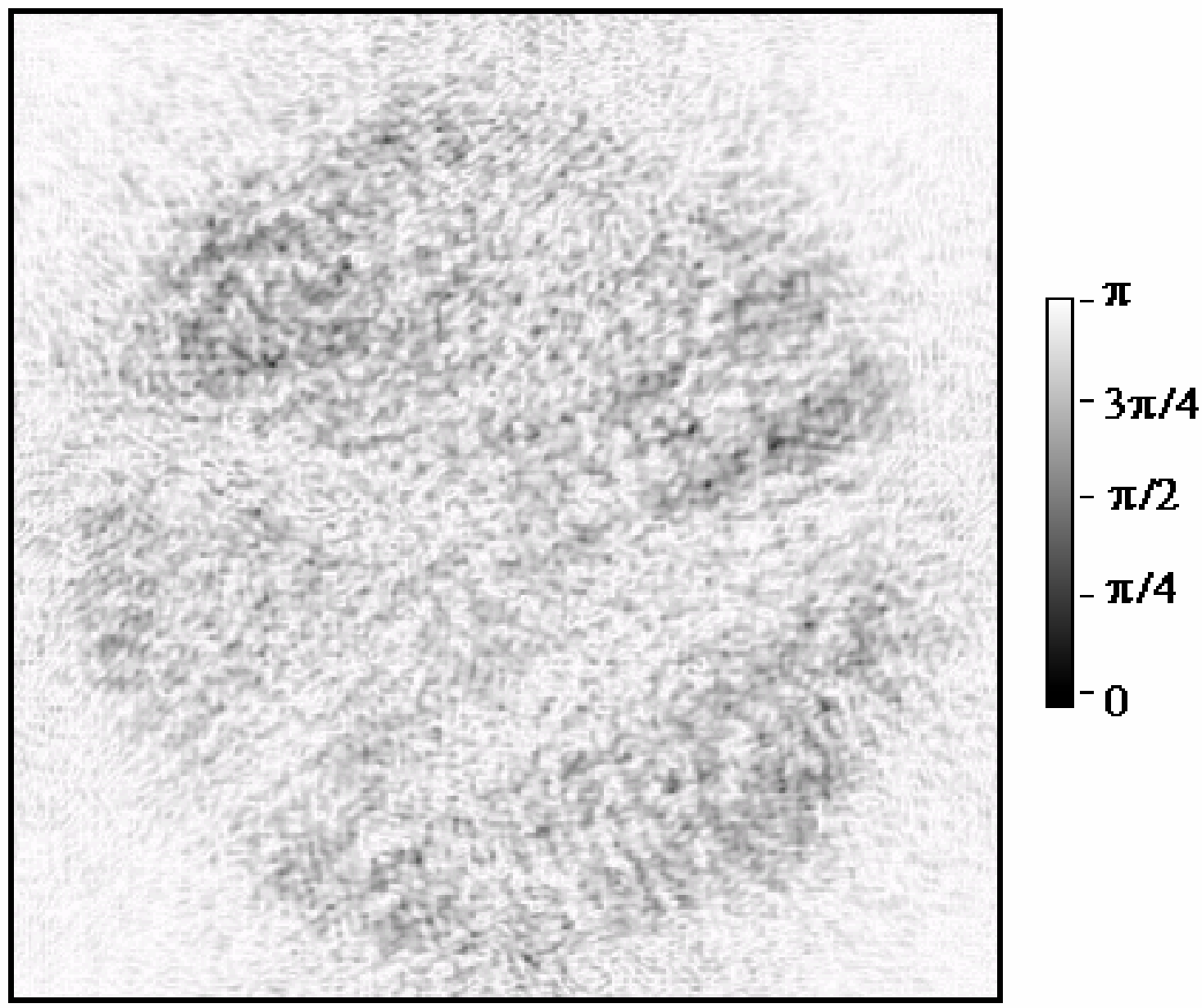

This result may be combined with B1.3 to yield $a(x, y)$, and the combined results may be subtracted directly from $\mathrm{B} 1.2$ to yield $c(x, y)$. 
Appendix B (Continued)

If the sample is now removed, and a final hologram acquired, its phase will be

$$
H_{\theta}(x, y)=k(a+b+c)
$$

where $a, b$, and $c$ are now constants, since there is no sample in place. By subtracting the results for $a(x, y)$ and $c(x, y)$, we are left with $b(x, y)$, which is of course the volume occupied by the cell itself. The value for $n(x, y)$ can be determined as well by simple division, thus showing any variation in refractive index across the profile of the cell; it is has been found that the nucleus possess a slightly different refractive index, and it may be that other organelles could be identified in this manner.

The most important aspect of this technique is the need for a long wavelength, since the numerical reconstructions provide solutions modulo $\pi$. As described in this thesis, it is possible to obtain reasonable results using two sources to simulate a longer wavelength, and this can be in principle extended to more sources for even longer range results. 


\section{Appendix C (Program documentation)}

Given below are the LabView front panels and block diagrams for three important programs used in these experiments: HistoData.vi, HCameraD.vi, and AAAHOLOEXPa.vi

HistoData.vi

Connector Pane

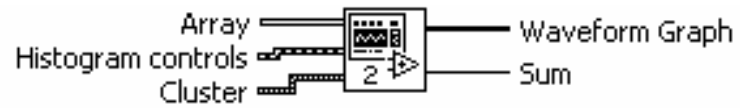

Front Panel

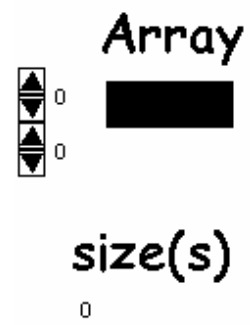

Histogram controls

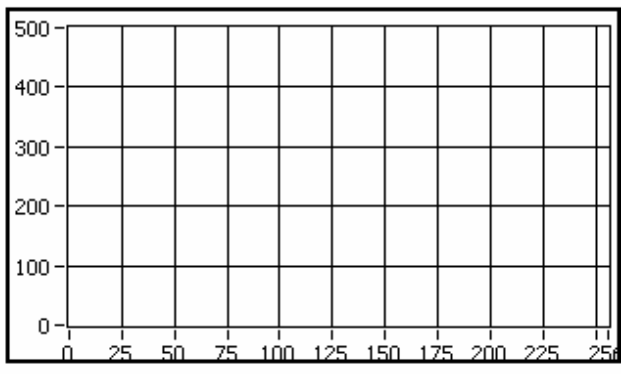

Normalize? Full H?

\section{Sum}

\section{Cluster}

\section{Black Ref}

四 0.00

\section{White Ref}

国 0.00 
Appendix C (Continued)

\section{Block Diagram}
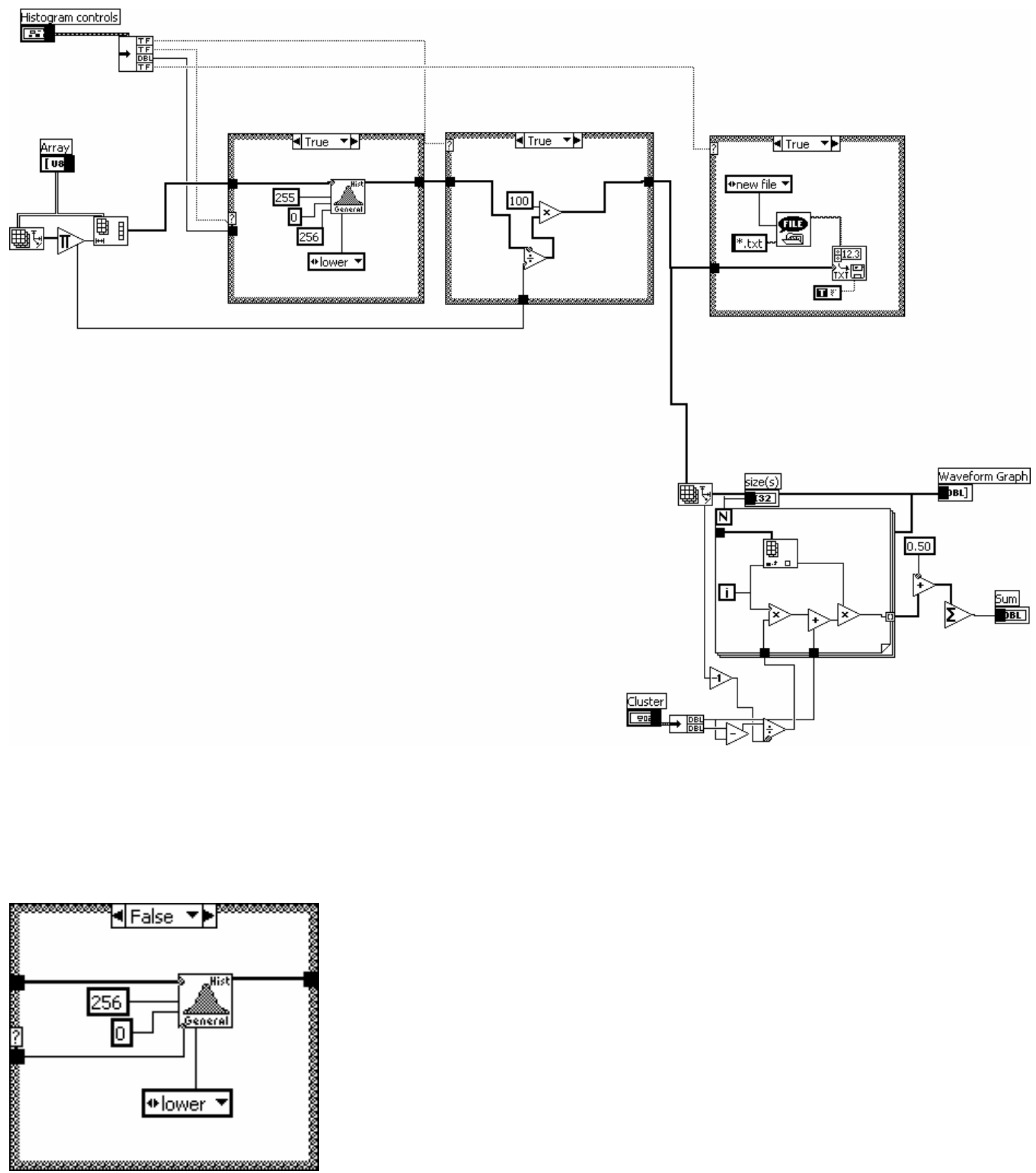
Appendix C (Continued)

HCameraD.vi

Connector Pane

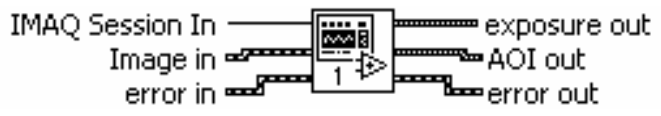

Front Pane I

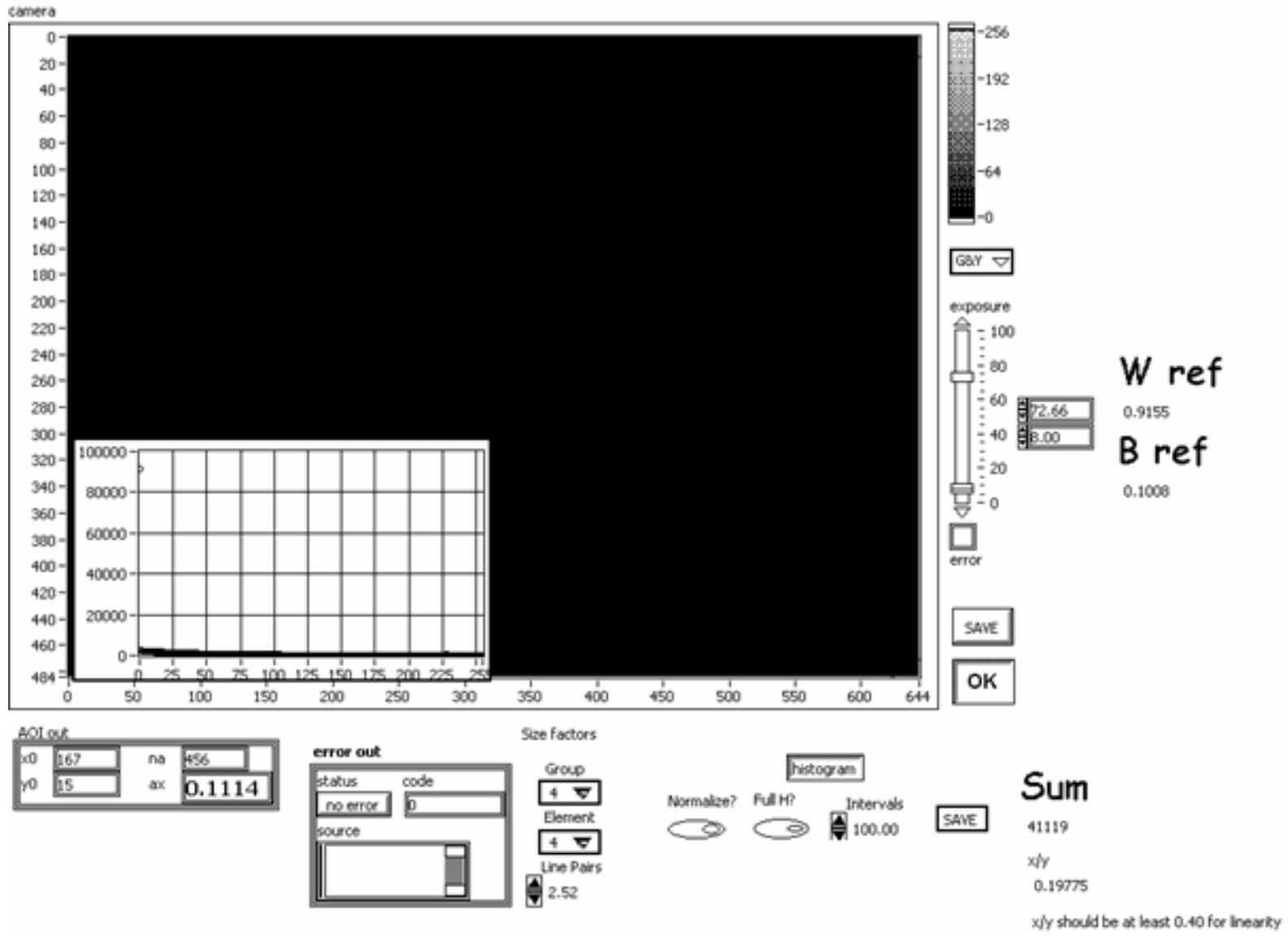


Appendix C (Continued)

\section{Block Diagram}
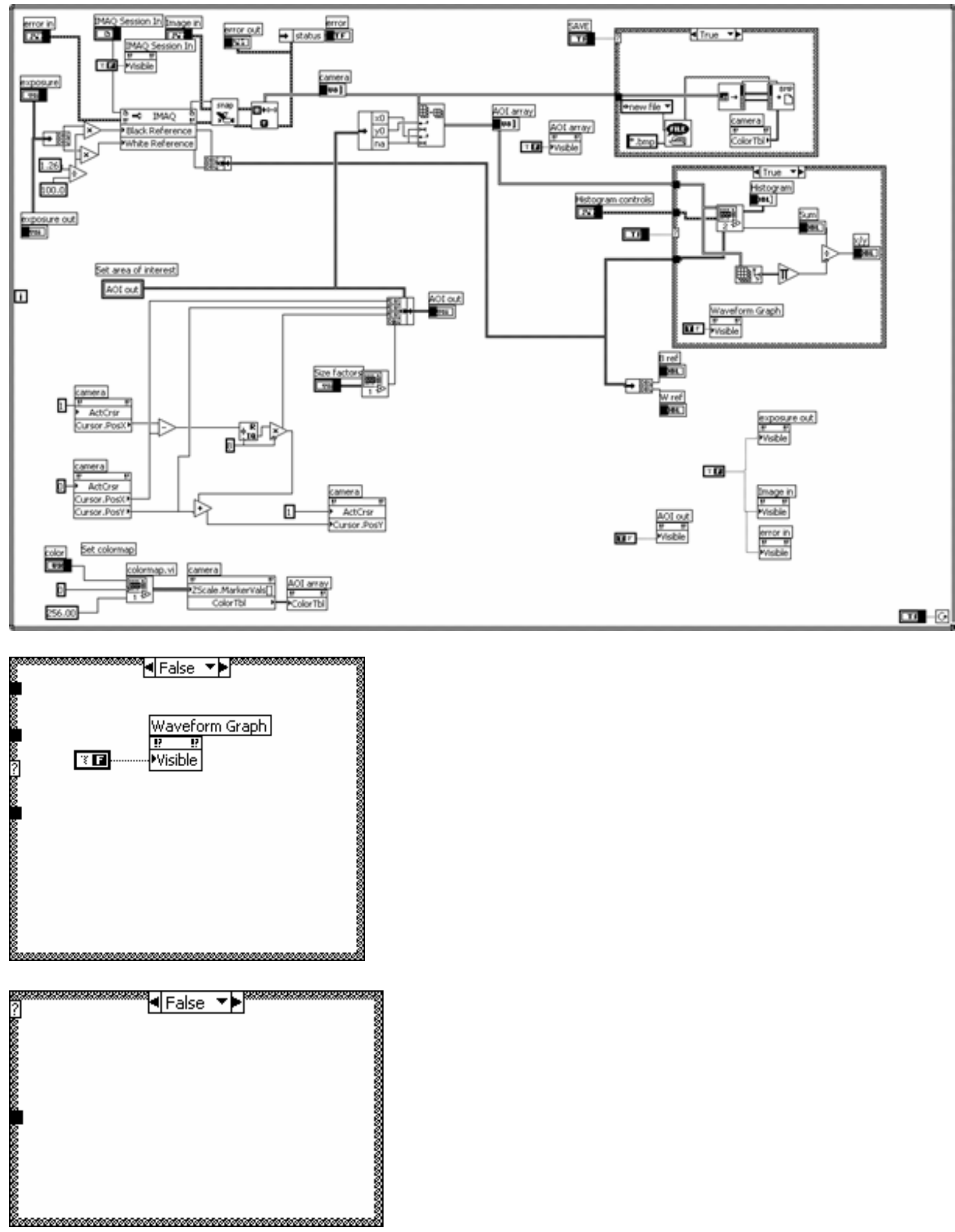
Appendix C (Continued)

AAAHOLOEXPa.vi

Connector Pane

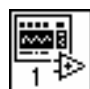

Front Panel
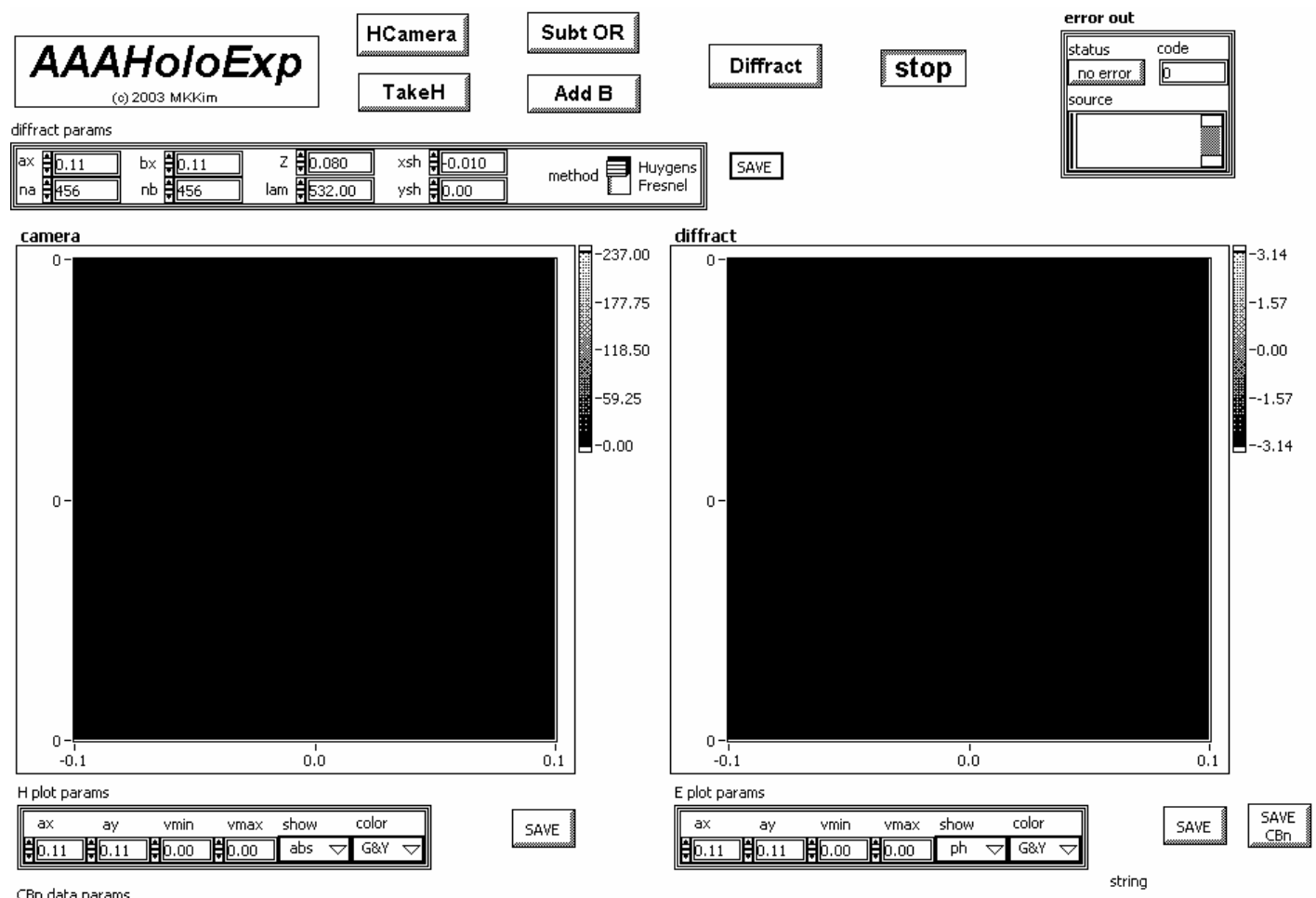

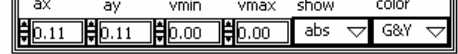

CBn data params

H:'AAAA HOLOEXP 12,02,03,AAAAHOLOEXPa.vi

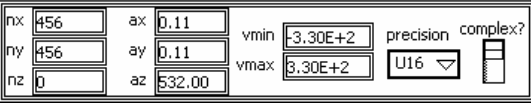




\section{Appendix C (Continued)}

\section{Block Diagram}
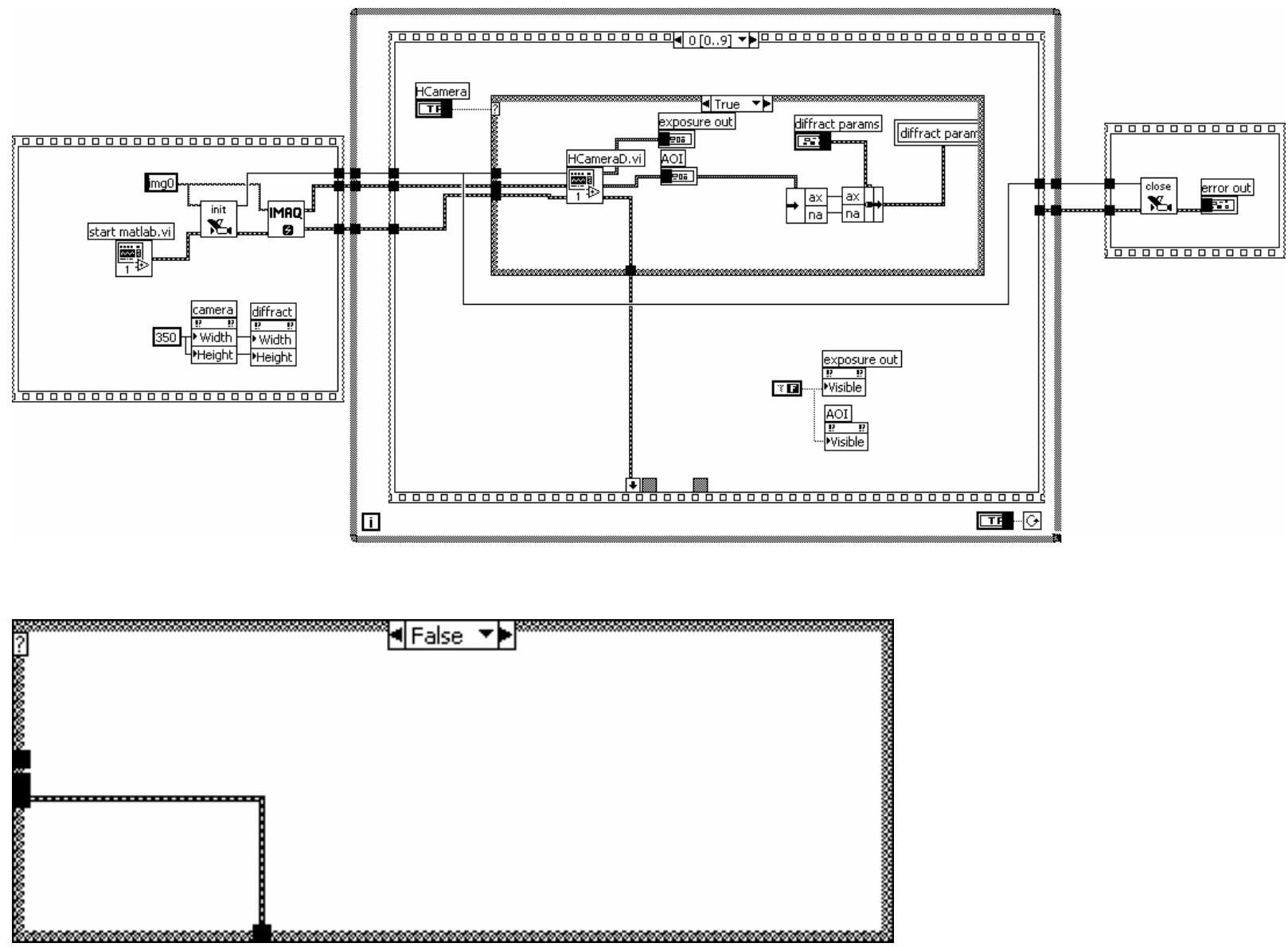


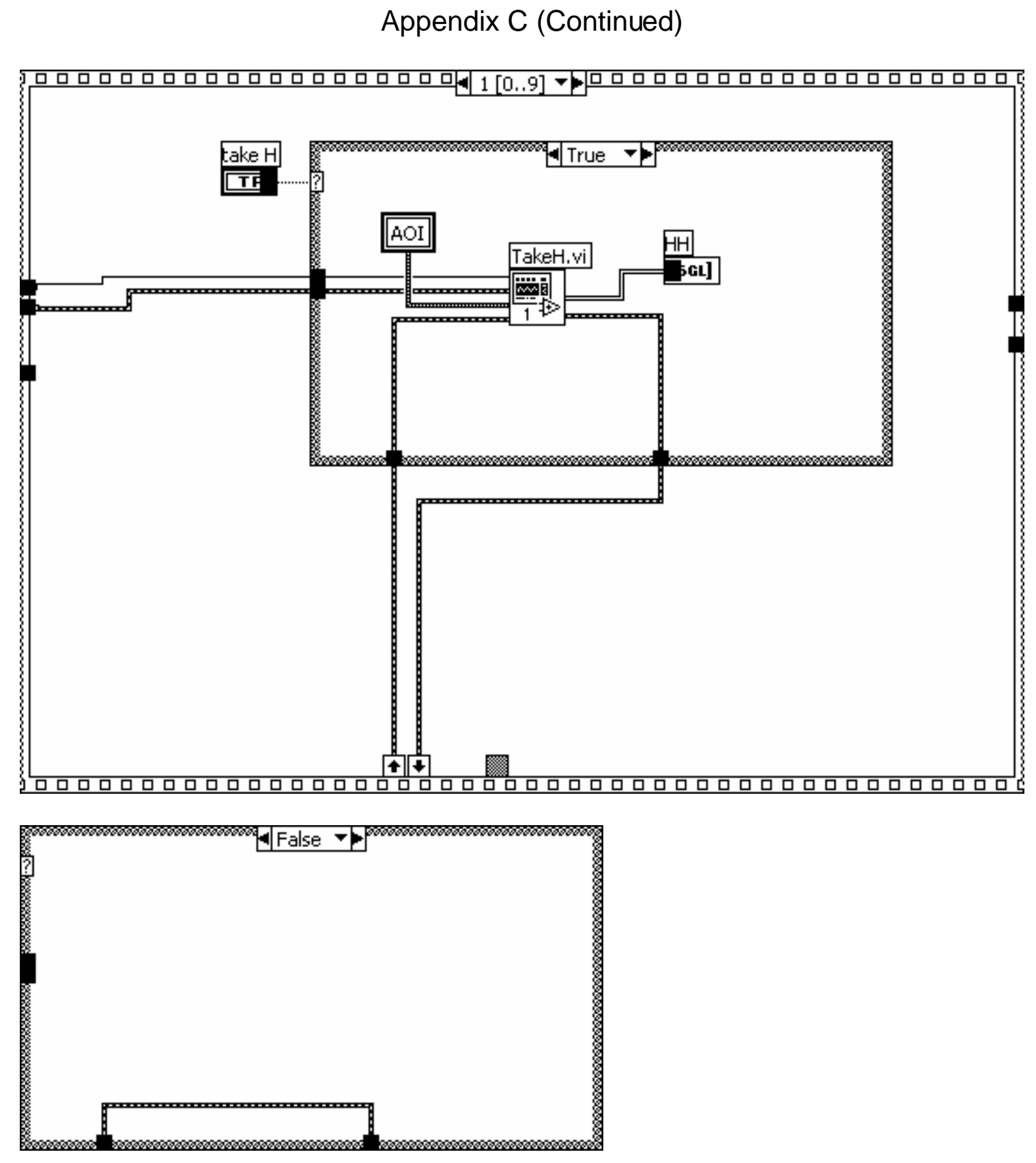



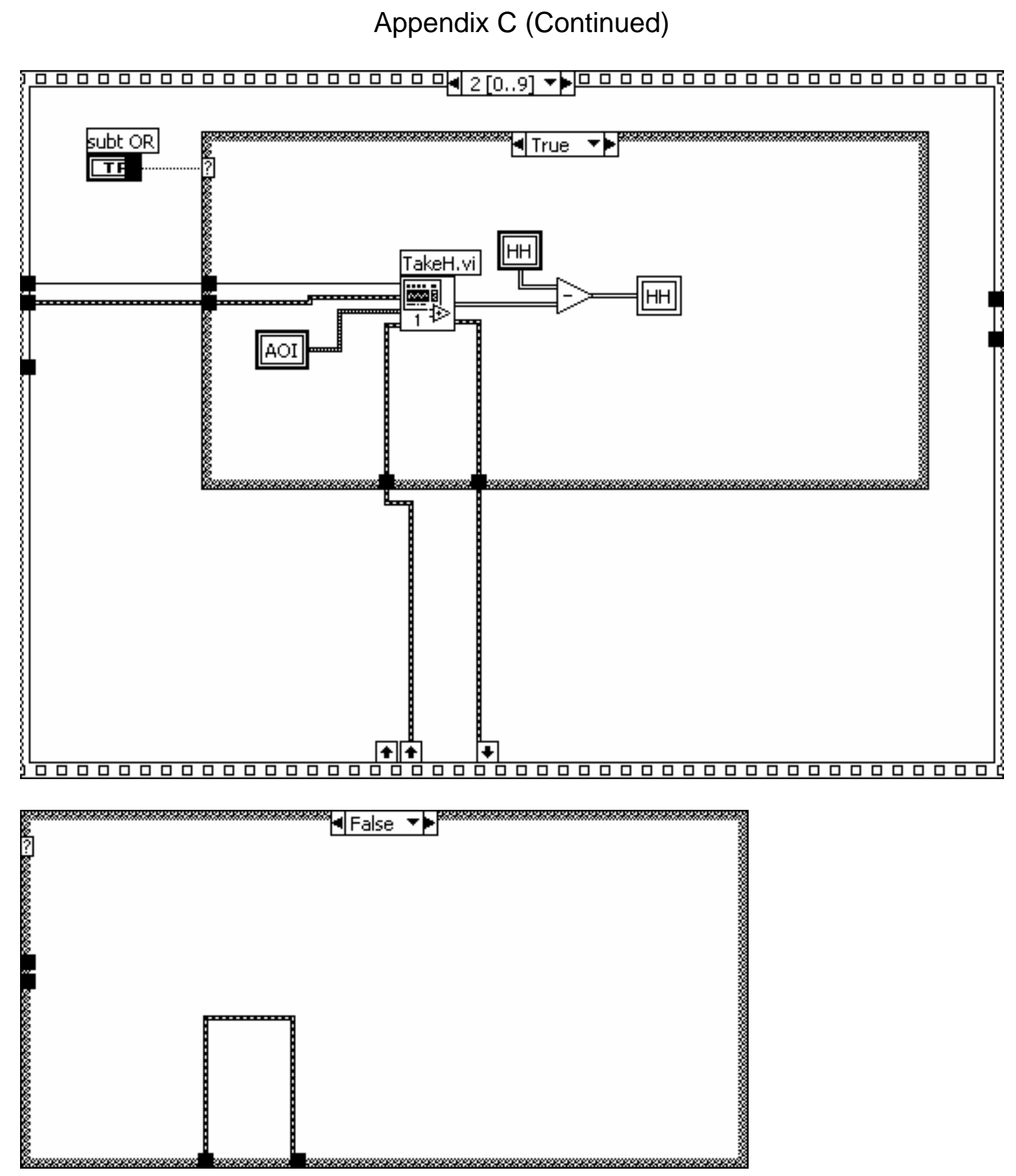

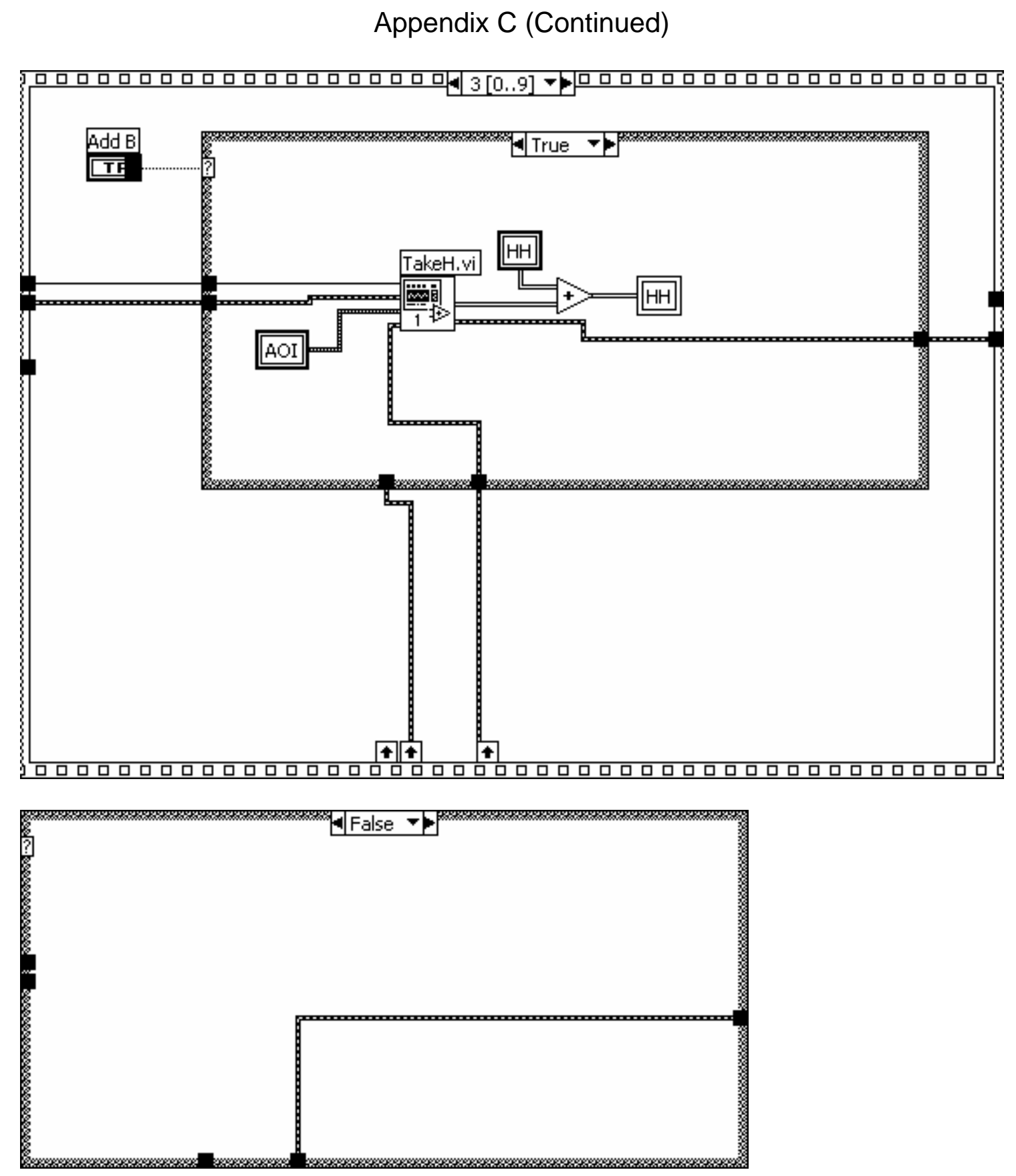
Appendix C (Continued)

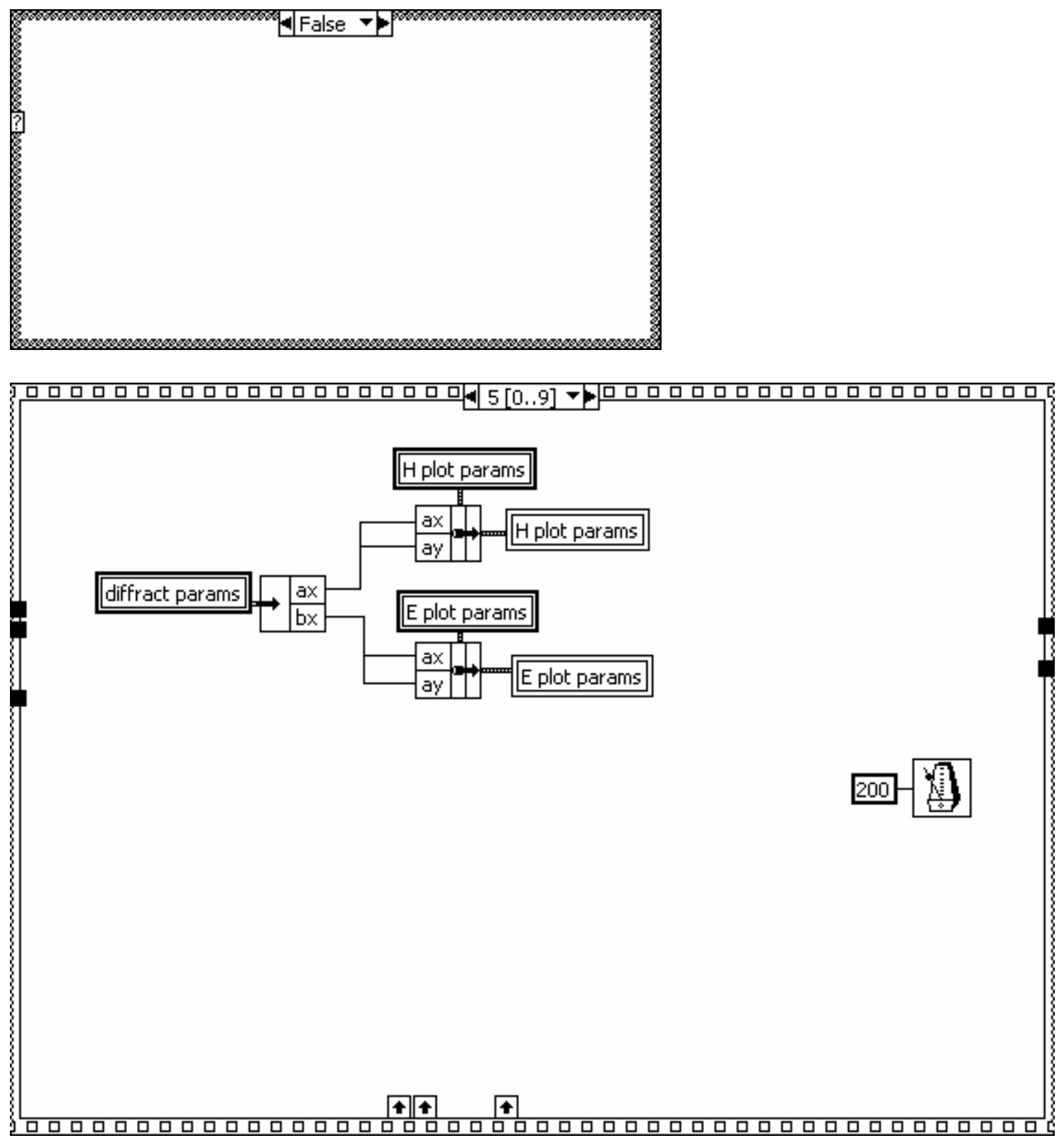


Appendix C (Continued)

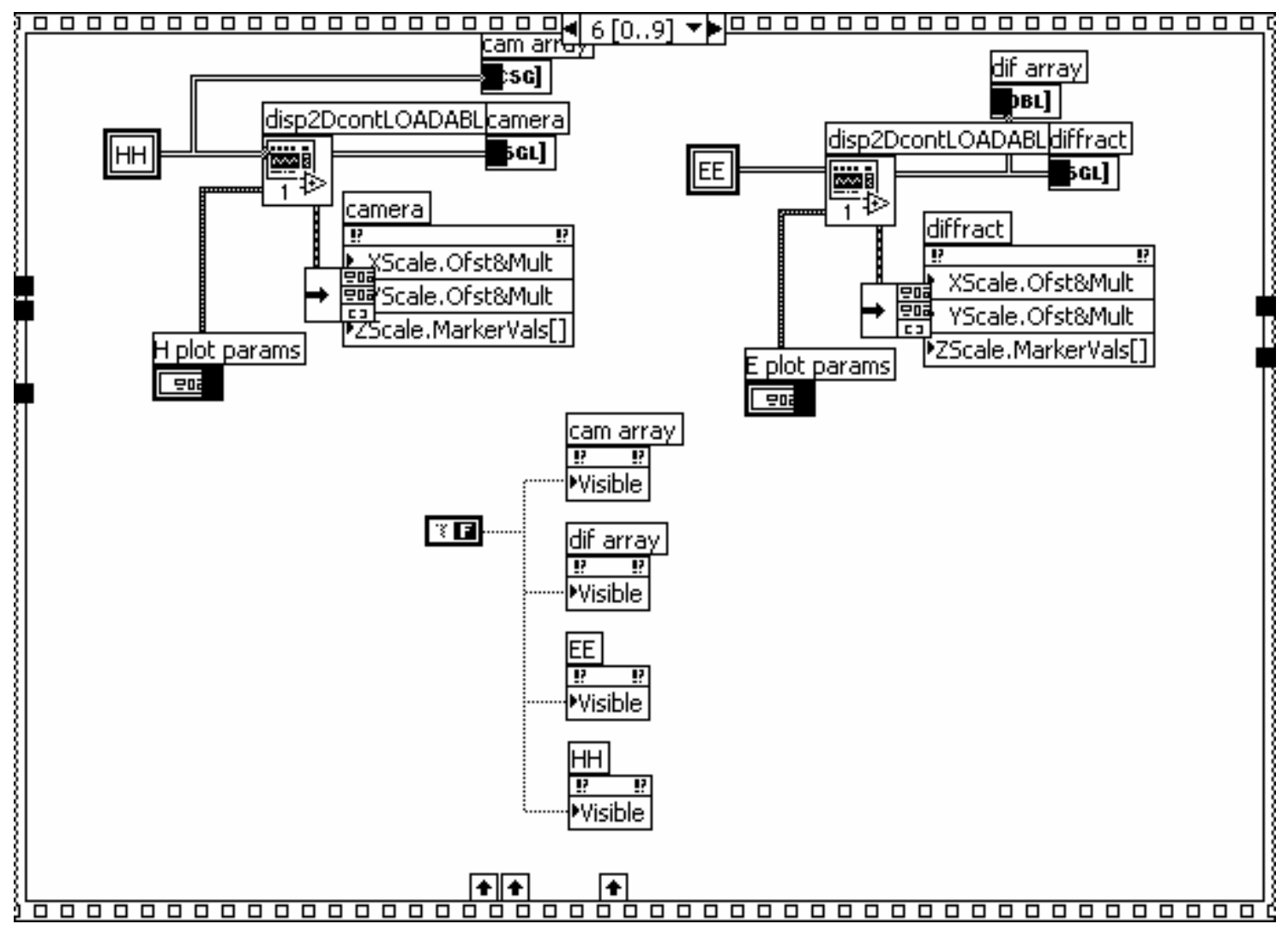


Appendix C (Continued)
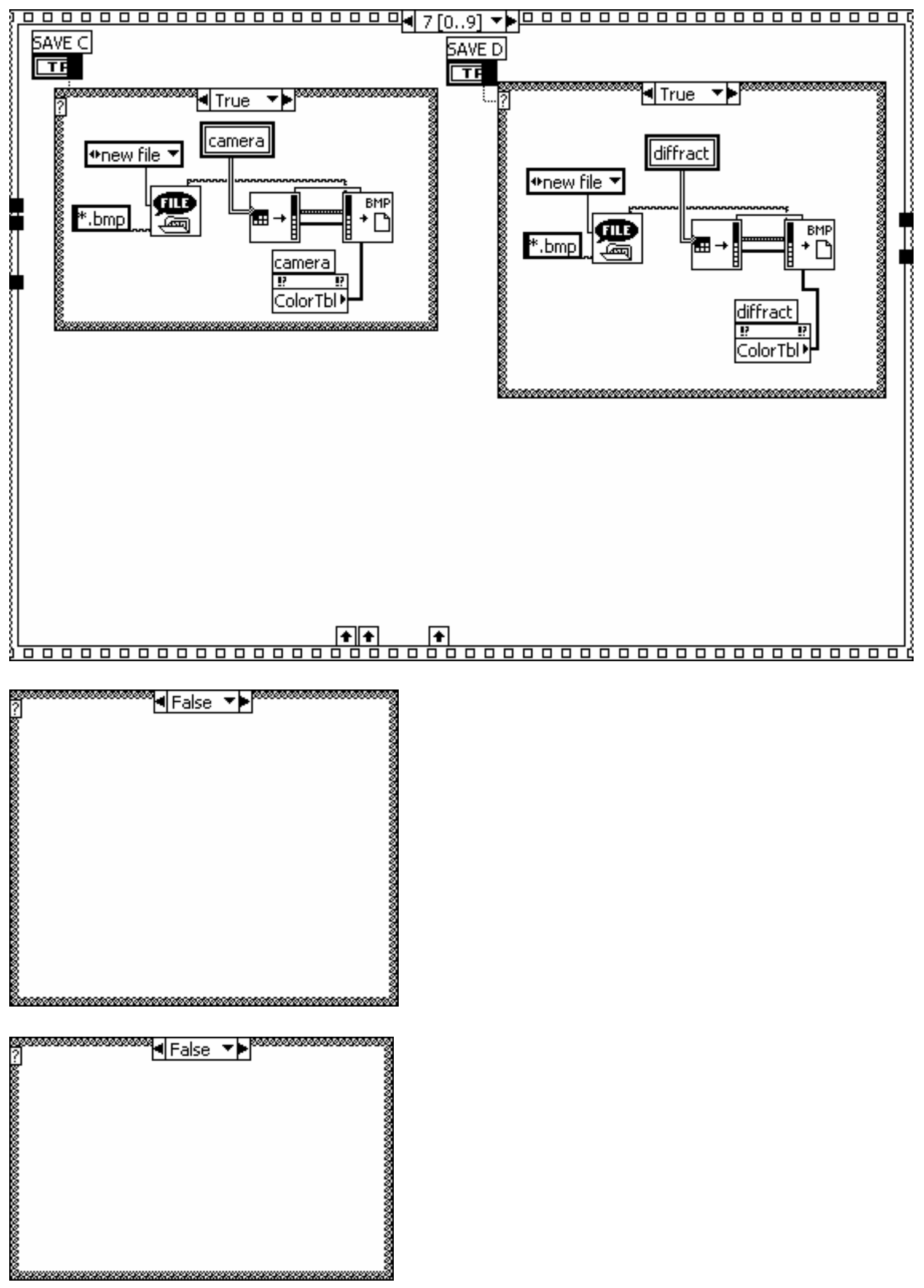


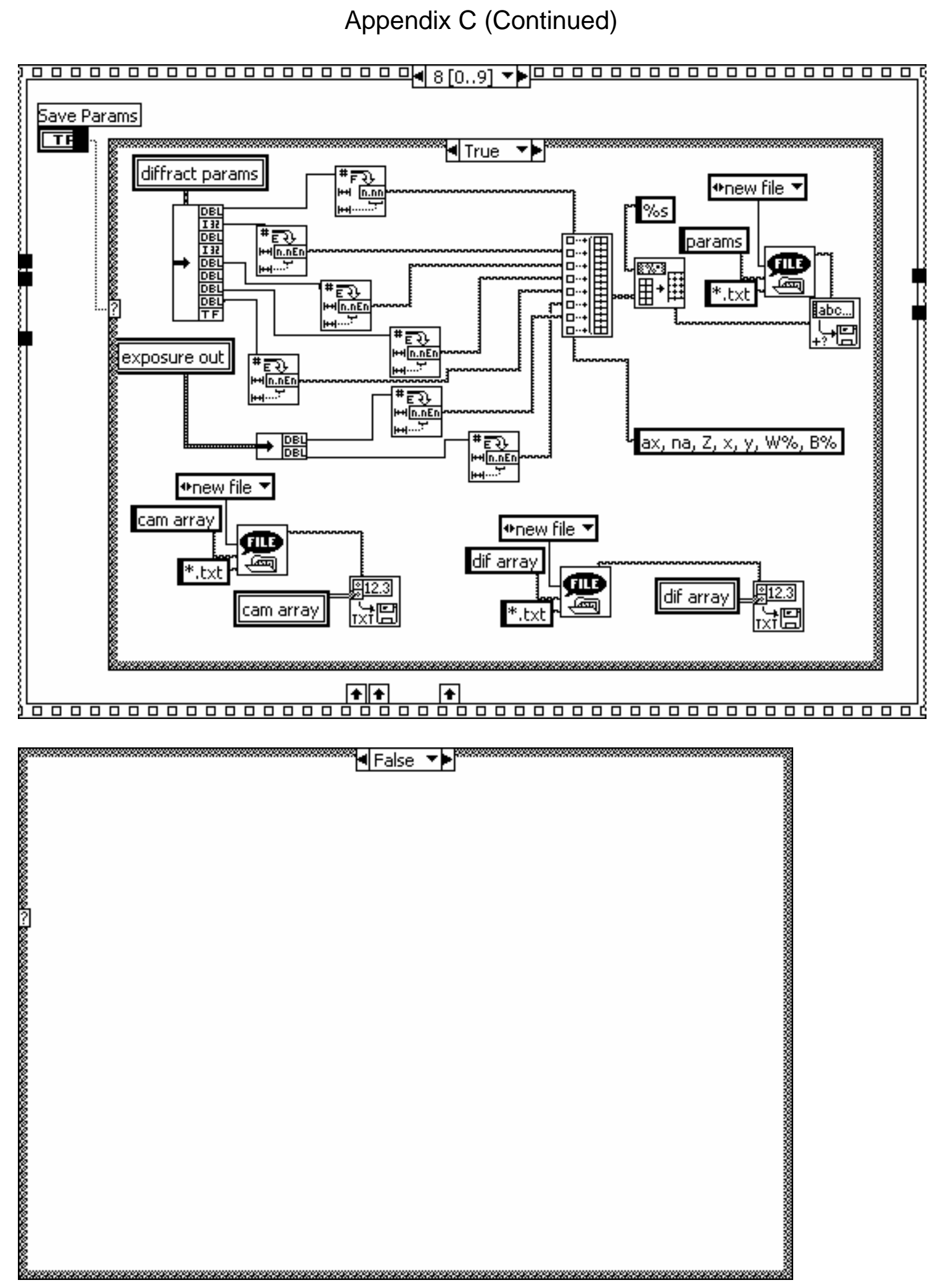




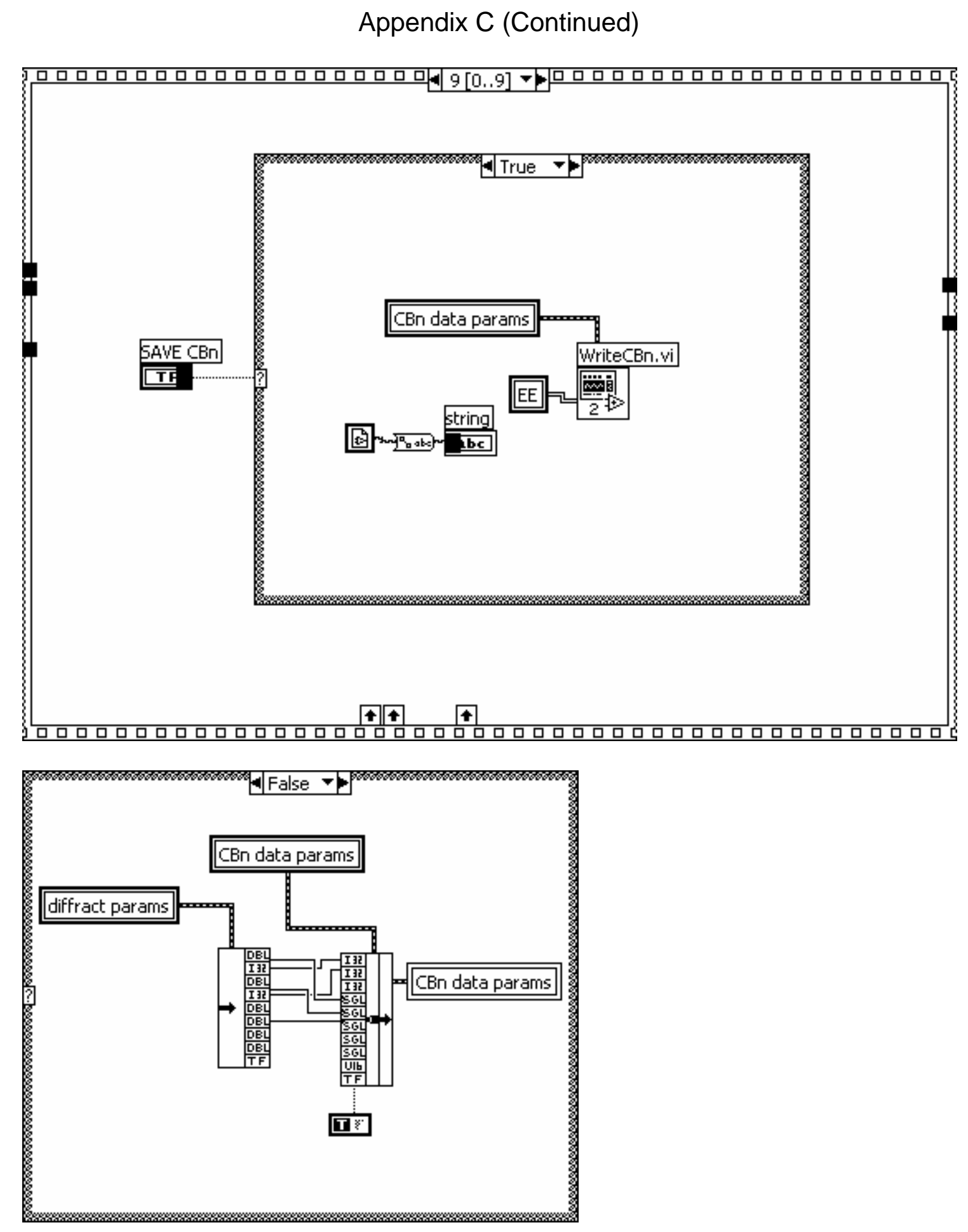


${ }^{1}$ While the first Leith-Upatnieks (off-axis) hologram was created shortly after the invention of the laser, their hologram actually used incoherent white light.

${ }^{2}$ The intrinsic properties of a light wave are amplitude, wavelength, and polarization. A hologram records the relative phase. The use of holos is therefore questionable, but it is conventional.

${ }^{3}$ Fig. 1 and 2 copyright M. K. Kim, 2003. Used with permission.

${ }^{4}$ The minimum angle between the object and reference beams to completely separate the two images is given by $\theta_{\text {min }}=\sin ^{-1} 3 \lambda f_{\text {max }}$, where $f_{\text {max }}$ is the maximum spatial frequency [16].

${ }^{5}$ The minimum distance was $Z_{\min }=\frac{a^{2}}{N \lambda}$, where $a$ was the width of the area being imaged.

${ }^{6}$ This calculation only needs to be performed once; since the hologram remains constant regardless of the $Z$ plane it in which its diffraction pattern is being evaluated, its FT remains constant as well.

${ }^{7}$ One experimenter (Gass [21]) wrote a program performing the convolution directly using the Huygens PTF, without the benefit of the FFT algorithms. He reported that a $40 \times 40$ pixel image took approximately one hour to complete.

${ }^{8}$ The Fourier window is a function which is multiplied by the function of interest; it allows us to consider the function of interest as extending to infinity, while only acquiring a finite data set.

${ }^{9} f_{\text {Nyquist }}=2 f_{\max }$, thus a fringe length of 2 pixels means the data is being sampled at a rate double the fringe rate.

${ }^{10}$ Neutral density filters produce the same attenuation for all wavelengths.

${ }^{11}$ Optical density is a measurement of attenuation of light through a filter. The intensity of light after passing through a filter is $10^{-O D}$ of its value before entering the filter.

${ }^{12} N A=\sin \left(\tan ^{-1}(d / 2 f)\right)$ where $d$ is the diameter and $\mathrm{f}$ the focal length of the lens.

${ }^{13}$ The CCD camera assigns a numerical value to each pixel based upon the voltage at that pixel. The voltage is based upon the incident light and can range between 0-1.26 V. The user can adjust the black and white reference values, thus narrowing or widening the range which is assigned as a particular intensity value. Anything more than $1.26 \mathrm{~V}$ is automatically assigned an intensity value of 255 .

${ }^{14}$ Astigmatism is the result of the lens being tilted with respect to the incoming light. Since the lens is not perfectly squared to the light, the light effectively sees two different curvatures as it passes through the lens. Thus there are, after the astigmatic lens, two optical axes that are not parallel.

${ }^{15}$ When pressed for a more precise value, Rosenfield asked the engineer in charge of production of the targets, who claimed a range of $+-5 \%$ for the depth of the film.

${ }^{16}$ This corrects fringes produced by the image processing. It does not resolve height differences greater than the wavelength, as does MWPIH.

${ }^{17}$ Approval to include these results in this thesis was given by the USF Insitutional Review Board on April 27, 2004. The letter of approval is on file with the USF Physics Department. 